Aproximações para a fila $M / G / s / r+G$

\title{
Gabriela Cantisano
}

\author{
DISSERTAÇÃO APRESENTADA \\ $\mathrm{AO}$ \\ Instituto DE MATEMÁticA E EstatísticA \\ DA \\ UnIVERSIDADE DE SÃO PAULO \\ PARA \\ OBTENÇÃO DO TÍTULO \\ $\mathrm{DE}$ \\ Mestre eM Cî̂nCIAS
}

\section{Programa: Estatística \\ Orientador: Prof. Dr. Marcos Nascimento Magalhães}

São Paulo, 3 de julho de 2009 



\section{Aproximações para a fila $M / G / s / r+G$}

Este exemplar corresponde à redação

final da dissertação/tese devidamente corrigida

e defendida por (Gabriela Cantisano)

e aprovada pela Comissão Julgadora.

Banca Examinadora:

- Prof. Dr. Marcos Nascimento Magalhães - IME-USP.

- Prof. Dr. Vanderlei da Costa Bueno - IME-USP.

- Prof. Dr. Graça Bressan - Poli-USP. 



\section{Agradecimentos}

Esta dissertação e trabalho de mestrado não teriam sido possível sem o total e irrestrito apoio de meu orientador, Prof. Dr. Marcos Nascimento Magalhães: agradeço imensamente toda a dedicação ofertada, o apoio, a paciência e os conhecimentos prestados durante esta jornada. Foram inúmeras situações de desafios e dificuldades, onde sempre pude contar com a colaboração e disseminação de conhecimento - que foram fator fundamental para a conclusão desta.

Dedico este trabalho aos meus pais, José Mário e Angela, aos meus irmãos Helen, Artur e Luciano e ao meu marido Thiago. Dedico também a todos meus amigos, aos quais sempre me apoiaram e contribuíram direta ou indiretamente para a realização deste sonho.

Agradeço também aos professores do Instituto de Matemática e Estatística da Universidade de São Paulo, em especial à Profa. Dra. Elizabeti Kira pelos valiosos conhecimentos passados, críticas, orientações e sugestões.

A todos os demais, que de alguma forma ajudaram-me, sou sincera em dizer-lhes: Muito obrigada! 


\section{Resumo}

Este trabalho estuda medidas de desempenho aproximadas em centrais de atendimento, apresentadas pelo modelo $M / G / s / r+G$. As aproximações são calculadas a partir do modelo $M / M / s / r+M(n)$.

Os resultados foram extendidos para o caso de mais de um tipo de cliente, apresentado pelo modelo $M / M^{i} / s / r+M^{i}(n)$.

Para dois casos particulares com 2 tipos de clientes, as aproximações citadas foram avaliadas numericamente e comparadas com os resultados de referência obtidos através de simulação. 


\section{Abstract}

We study approximations for performance measures of call centers, represented by $M / G / s / r+G$ queueing model. We use the measures computed in the $M / M / s / r+M(n)$ queueing model.

The results were extended for more then one type of customer, represented by $M / M^{i} / s / r+M^{i}(n)$ queueing model.

For two particular cases with two types of customers, the mentioned approaches were numerically evaluated and compared with the results of reference obtained by simulation. 


\section{Sumário}

$\begin{array}{lc}\text { Lista de Figuras } & \text { ix }\end{array}$

Lista de Tabelas

1 Introdução $\quad 1$

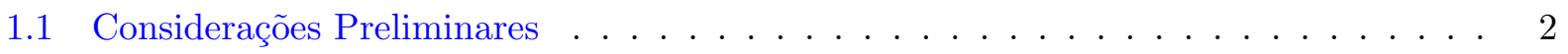

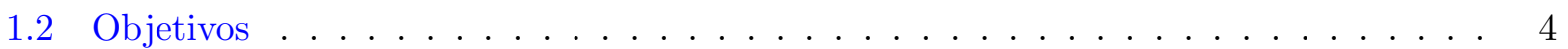

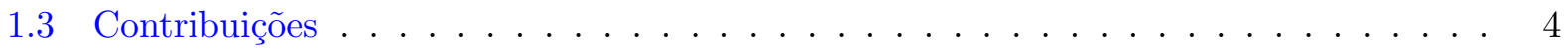

1.4 Organização do Trabalho . . . . . . . . . . . . . . . . . . . . 4

2 Aproximação de fila para Centrais de Atendimento $\quad 5$

2.1 Introdução . . . . . . . . . . . . . . . . . . . . . . . 5

2.2 Aproximação Markoviana para Abandonos . . . . . . . . . . . . . . . . 8

2.3 Aproximação Markoviana para Tempos de Atendimento . . . . . . . . . . . . . . . 11

2.4 Distribuição Estacionária do Modelo Markoviano . . . . . . . . . . . . . . . . . 13

2.4.1 Distribuição Estacionária do Processo de Nascimento e Morte . . . . . . . . . 13

2.4.2 Probabilidade de um Cliente ser Atendido ou Abandonar . . . . . . . . . . . 14

2.4.3 Tempo de Espera para Clientes que são Atendidos . . . . . . . . . . . . . . 16

2.4.4 Probabilidade de um Cliente Abandonar . . . . . . . . . . . . . . . . . 18

2.5 Descrição do algoritmo e resultados numéricos . . . . . . . . . . . . . . . . . 19

3 Central de Atendimento com mais de um tipo de cliente. 25

3.1 Introdução . . . . . . . . . . . . . . . . . . . 25

3.2 Modelo $M / M^{i} / s / r+M^{i}(n) \ldots \ldots \ldots \ldots \ldots \ldots \ldots$

3.3 Medidas de Desempenho $-M / M^{i} / s / r+M^{i}(n) \ldots \ldots \ldots \ldots$

4 Casos particulares $\quad 43$

4.1 Introdução . . . . . . . . . . . . . . . . . . . . . . . 43

4.2 Caso $M / M^{i} / 1 / 1+M^{i}(n), i=2 \ldots \ldots \ldots \ldots \ldots \ldots \ldots$

4.2.1 Medidas de desempenho caso $M / M^{i} / 1 / 1+M^{i}(n), i=2 \ldots \ldots$. . . . 50

4.2 .2 Validação . . . . . . . . . . . . . . . . . . . . . . . . 54

4.2 .3 Resultados Numéricos e Análises . . . . . . . . . . . . . . . . . . 58

4.3 Caso $M / M^{i} / 2 / 3+M^{i}(n), i=2 \ldots \ldots \ldots \ldots \ldots \ldots \ldots \ldots$ 
5 Conclusões $\quad \mathbf{6 5}$

5.1 Considerações Finais . . . . . . . . . . . . . . . . . . . 65

$\begin{array}{ll}\text { A Anexo } & 67\end{array}$

A.1 Caso $M / M^{i} / 2 / 3+M^{i}(n), i=2 \ldots \ldots \ldots \ldots \ldots \ldots \ldots$

A.1.1 Fluxo de estados . . . . . . . . . . . . . . . . . . 67

A.1.2 Equações de balanço . . . . . . . . . . . . . . . . . . . . . . . . . . . . . . . . .

A.1.3 Medidas de desempenho . . . . . . . . . . . . . . . . 76

A.2 Algoritmo . . . . . . . . . . . . . . . . . . . 80

A.2.1 Simulação dois tipos de clientes . . . . . . . . . . . . . 80

Referências Bibliográficas $\quad 93$ 


\section{Lista de Figuras}

1.1 Central de Atendimento. . . . . . . . . . . . . . . . . . . 3

2.1 Contagem de clientes. . . . . . . . . . . . . . . . . . 9

3.1 Sistema com $s$ servidores, sala de espera com $r$ clientes de $m$ tipos. . . . . . . . . 26

3.2 Reposicionamento de clientes após fim de serviço . . . . . . . . . . . . . . . . 27

3.3 Reposicionamento de clientes após abandono. . . . . . . . . . . . . . . 28

3.4 Fluxo caso 1. . . . . . . . . . . . . . . . . . . . . . . . . 30

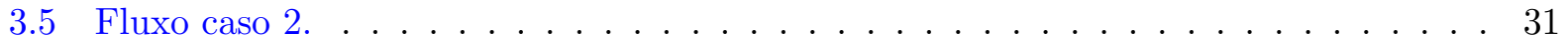

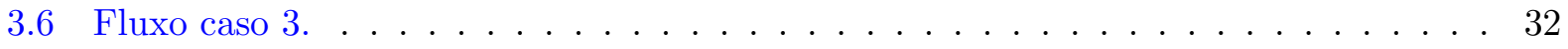

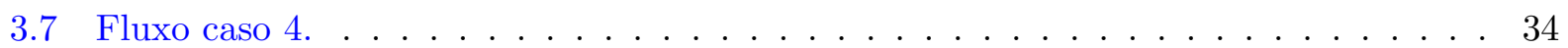

3.8 Tipo e posição de clientes na fila. . . . . . . . . . . . . . . . 36

4.1 Modelo $M / M^{i} / 1 / 1+M^{i}(n)(i=2) \ldots \ldots \ldots \ldots \ldots \ldots$

4.2 Fluxo do modelo $M / M^{i} / 1 / 1+M^{i}(n)(i=2) \ldots \ldots \ldots \ldots$. . . . . . . 45

4.3 Modelo $M / M^{i} / 2 / 3+M^{i}(n)(i=2) \ldots \ldots \ldots \ldots \ldots \ldots$. . . . . . . . 61

4.4 Fluxo de alguns estados do modelo $M / M^{i} / 2 / 3+M^{i}(n)(i=2) \ldots \ldots$. . . . . 62 


\section{Lista de Tabelas}

2.1 Comparação das simulações em $M / M / r / s+G \ldots \ldots \ldots$

2.2 Comparação das simulações em $M / G / s / r+E_{2} \ldots \ldots \ldots \ldots$. . . . . . . . . 12

2.3 Comparação da simulação do modelo de fila $M / M / 100 / 200+M \ldots$. . . . . . . . . 21

2.4 Comparação da simulação do modelo de fila $M / E_{2} / 100 / 200+E_{2}$. . . . . . . . . . 22

2.5 Comparação da Aproximação do modelo de fila $M / M / 100 / 200+M$. . . . . . . . . 23

2.6 Comparação da Aproximação do modelo de fila $M / E_{2} / 100 / 200+E_{2}$. . . . . . . . . 24

4.1 Validação da silmulação para filas com dois tipos de clientes. . . . . . . . . . . . 56

4.2 Validação da aproximação para filas com dois tipos de clientes. . . . . . . . . . . 57

4.3 Resultados cenários 1 e 2, para o modelo $M / M^{i} / 1 / 1+M^{i}(n)(i=2)$. . . . . . . 59

4.4 Resultados cenários 3 e 4, para o modelo $M / M^{i} / 1 / 1+M^{i}(n)(i=2) \ldots \ldots$. . . . 60

4.5 Resultados cenário 1, para o modelo $M / M^{i} / 2 / 3+M^{i}(n)(i=2) \ldots \ldots$. . . . . 63

4.6 Resultados cenário 2, para o modelo $M / M^{i} / 2 / 3+M^{i}(n)(i=2) \ldots \ldots$. . . . . 64

A.1 Fluxo $M / M^{i} / 2 / 3+M^{i}(n)(i=2)($ parte 1$) \ldots \ldots \ldots \ldots$. . . . . . . 68

A.2 Fluxo $M / M^{i} / 2 / 3+M^{i}(n)(i=2)($ parte 2$) \ldots \ldots \ldots \ldots$. . . . . . . 69

A.3 Fluxo $M / M^{i} / 2 / 3+M^{i}(n)(i=2)($ parte 3$) \ldots \ldots \ldots \ldots$ 


\section{Capítulo 1}

\section{Introdução}

Por Central de Atendimento entende-se a integração cliente/empresa, composta pelo atendimento, soluções e interface entre consumidores e fornecedor (ou empresa de serviços/venda de mercadorias em geral). Seu objetivo, além do simples contato cliente/empresa é o gerenciamento e administração do relacionamento pré ou pós-vendas entre as partes envolvidas. A busca por melhor nível de atendimento, aliado à necessidade de diferenciais competitivos e ao atual aumento na demanda, levou o país nos últimos anos a ter expressivo crescimento no setor de Centrais de Atendimento.

Segundo estudo feito pela IDC (em inglês, International Data Corporation), no Brasil em 2006, registrou-se um crescimento de $20 \%$ no setor de serviços de Centrais de Atendimento e em 2007 o crescimento foi de 17\%. Estima-se que, principalmente em função da nova regulamentação brasileira no setor (em vigência a partir de dez/2008), o investimento em Centrais de Atendimento deve-se manter. Segundo as novas regras, o tempo de espera não pode se estender além de 1 minuto, sob o risco de altas penalidades (multas de até 3 milhões de reais, por infração). Como as Centrais de Atendimento não estão adequadas à nova regulamentação e também ao nível de serviço esperado pelos clientes, em 2009 devem ocorrer novas oportunidades de trabalho, investimentos em infraestrutura/equipamentos e práticas (metodologias e pesquisas) no setor.

Para Gans, Koole e Mandelbaum em [6], uma Central de Atendimento ideal, com um certo número de atendentes poderia receber milhares de chamadas por hora, mantendo cerca de $95 \%$ dos seus atendentes ocupados. Além disso, todos os clientes que buscam contato com a Central de Atendimento, conseguem acessar o sistema e, aproximadamente, metade daqueles que entram no sistema recebem atendimento imediato, fazendo com que a taxa de abandono fique em torno de $2 \%$.

O perfil dos clientes atendidos vem mudando ano a ano. O conhecimento sobre os produtos aumentou e, assim, os clientes se tornam mais exigentes, querem um serviço de qualidade com solução de problemas de forma imediata. Dessa forma, as empresas são pressionadas a fornecer um serviço cada vez mais eficiente e rápido. 
Segundo [6], uma Central de Atendimento raramente mantém um alto nível de eficiência e qualidade ao mesmo tempo, o que torna seu comportamento foco de vários estudos teóricos, como pode ser visto em [12].

\subsection{Considerações Preliminares}

Uma Central de Atendimento ou call center é basicamente uma central telefônica com vários atendentes em que a espera pelo atendimento pode ocorrer.

Uma fila é formada quando clientes ligam e todos os servidores estão ocupados. Como os clientes estão impossibilitados de receber atendimento imediato, eles entram em fila para aguardar o término do atendimento de algum cliente que está sendo servido. Nessa espera pode haver abandono.

Uma Central de Atendimento pode ser analisada como um modelo de fila, como pode ser visto em [8], [9] e [13].

O esquema básico de funcionamento é descrito na Figura 1.1.

Observe que existem três eventos básicos:

- Chegada de um cliente.

- Término de um serviço.

- Abandono do cliente.

O estudo das características de uma Central de Atendimento, pode muitas vezes ser analiticamente intratável. Uma razão pela qual Centrais de Atendimento são complicadas de analisar é que frequentemente elas envolvem múltiplos locais e grupos de agentes, diferentes habilidades, e servindo múltiplas classes de clientes com necessidades diferentes. Outra razão é que os clientes que estão esperando na fila podem abandonar. Além disso, as distribuições tanto do tempo de atendimento quanto do tempo de abandono, frequentemente, não são próximas da distribuição Exponencial, tornando inapropriada a aplicação direta do modelo Markoviano simples (ver [1], [3]). Além disso, como pode ser visto em [5], a taxa de chegada de clientes em uma Central de Atendimento pode não ser constante ao longo do dia, tornando o modelo ainda mais complexo.

Representando as Centrais de Atendimento como modelos de fila, consideramos um número de servidores $s$, uma sala de espera de tamanho $r$, e chegadas sendo um processo de Poisson e taxa constante $\lambda$. Os clientes são atendidos segundo alguma distribuição de probabilidade com média $1 / \mu$ e podem abandonar a espera em fila, seguindo também uma paciência aleatória, com taxa que 


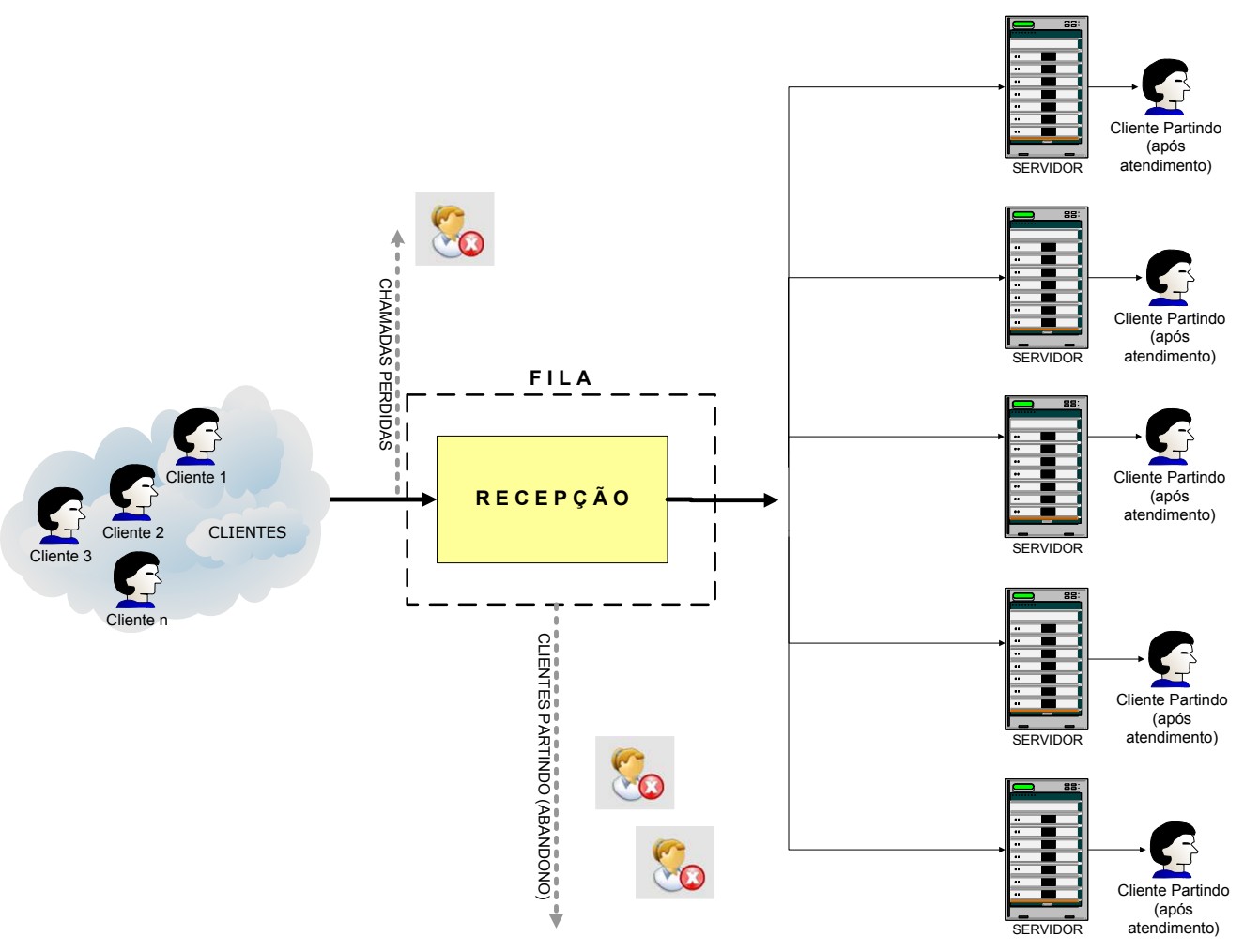

Figura 1.1: Central de Atendimento.

pode ser função de sua posição na fila e do tempo de espera até aquele momento. Desta forma o tempo de abandono indica a tolerância do usuário a esperar pelo serviço. Usaremos as palavras paciência e tempo de abandono com interpretações similares.

Em um segundo momento, vamos atribuir tipos para os clientes que chegam no sistema, ou seja, a taxa de chegada no sistema se mantém mas assim que o cliente entra, ele é classificado. Os vários tipos de clientes se distinguem pela distribuição de probabilidade do abandono e/ou atendimento.

Não serão considerados os casos de servidores com habilidades diferentes e taxas de chegada variando ao logo do tempo. Como em geral a taxa de chegada de clientes ao longo do dia não é constante, vamos estudar os períodos de pico.

A notação utilizada para representar uma fila será $A / B / s / r+C$, em que,

- A - distribuição do tempo entre chegadas;

- B - distribuição do tempo de serviço; 
- s - número de servidores;

- r - número de lugares na sala de espera;

- C - distribuição do tempo de abandono.

Distribuições utilizadas (ver [10].):

- $G$ : distribuição geral;

- $M$ : distribuição Exponencial;

- $E_{2}$ : distribuição Erlang2.

\subsection{Objetivos}

O objetivo deste trabalho é apresentar os resultados desenvolvidos por Whitt em [15], que aproximou o modelo de fila $M / G / s / r+G$ pelo modelo totalmente Markoviano $M / M / s / r+M(n)$. Whitt desenvolveu a aproximação para clientes de um tipo, considerando todos os clientes no sistema com taxas de abandono e atendimento iguais. Neste trabalho desenvolvemos a aproximação considerando mais de um tipo de cliente, para aprimorar a aproximação desenvolvida anteriormente.

\subsection{Contribuições}

A principal contribuição deste trabalho é analisar, através da aproximação Markoviana, o comportamento de uma Central de Atendimento com características gerais, contemplando em uma mesma fila, $m$ diferentes características de clientes.

\subsection{Organização do Trabalho}

No Capítulo 2, apresentamos as aproximações desenvolvidas por Whitt em [15] e seus resultados numéricos.

No Capítulo 3, apresentamos o modelo de fila com mais de um tipo de cliente. Fazemos as aproximações para o modelo $M / G^{i} / s / r+G^{i}$ a partir do modelo $M / M^{i} / s / r+M^{i}(n)(i=2)$.

No Capítulo 4, apresentamos as equações de balanço, as medidas de desempenho para os casos particulares $M / M^{i} / 1 / 1+M^{i}, M / M^{i} / 2 / 3+M^{i}(i=2)$ e seus resultados numéricos.

Finalmente, no Capítulo 5 discutimos as conclusões obtidas neste trabalho e possibilidades para pesquisa futura. 


\section{Capítulo 2}

\section{Aproximação de fila para Centrais de Atendimento}

\subsection{Introdução}

Neste Capítulo apresentamos os resultados obtidos por Ward Whitt em [15]. Whitt parte de um modelo geral de central de atendimento $M / G / s / r+G$ e obtém aproximações para diversas medidas de desempenho através do modelo $M / M / s / r+M(n)$, o qual possui solução analítica.

Para uma Central de Atendimento com $s$ servidores, sala de espera de tamanho $r$, chegadas com distribuição Poisson e que:

- trabalha com apenas uma classe de clientes;

- possui apenas um grupo de agentes;

- não permite aos clientes obterem informação sobre a fila e;

- apresenta disciplina de atendimento FCFS (First-Come-First - Served), ou seja, os clientes são atendidos de acordo com sua ordem de chegada;

é comumente acordado, veja [2] e [12], que sua dinâmica pode ser bem descrita por um modelo da forma $M / G / s / r+G$. Essa fila considera o processo de chegadas seguindo um processo de Poisson $(M)$, com atendimento de forma independente e identicamente distribuídos (iid) de acordo com uma distribuição geral $G$ (primeiro $G$ ) e com tempo de abandonos descritos por outra distribuição geral $G$ (segundo $G$ ) também iid. Note que centrais de atendimento em que a fila é invisível para os clientes é razoável supor que os tempos de abandono são iid.

O modelo $M / G / s / r+G$ é bastante geral o que faz com que não seja simples tratá-lo de maneira analítica. De fato, propriedades e resultados teóricos foram derivados apenas para alguns casos particulares como o $M / M / s / r+M$ (tempo de serviço e de abandono, Exponenciais), vide [14] e [7].

Dessa forma, uma alternativa é estudar as principais características dos modelos e estimar métricas de desempenho de centrais de atendimento utilizando aproximações e/ou simulações. O estudo via simulação é intensivo computacionalmente, inviabilizando em muitos casos a aplicação 
prática da solução. Esta é uma motivação importante para o uso de aproximações.

São apresentadas nas próximas seções aproximações baseadas no modelo $M / M / s / r+M(n)$ para as principais métricas de desempenho de centrais de atendimento que seguem o modelo $M / G / s / r+G$. Essas aproximações podem ser utilizadas como alternativas aos modelos de simulação para o estudo das principais características das centrais de atendimento.

Vamos adotar a seguinte notação:

- $S$ : evento em que um cliente típico, após entrar no sistema, é servido;

- A: evento em que um cliente típico, após entrar no sistema, abandona antes do início do serviço;

- $W$ : tempo de espera de um cliente típico que entra no sistema, em regime estacionário, até o início de serviço ou abandono (aquele que ocorrer primeiro);

- $N$ : número de clientes no sistema, em estado estacionário, em um tempo arbitrário;

- $Q \equiv \max \{0, N-s\}$ : número de clientes na fila em um instante arbitrário no estado estacionário.

Com a finalidade de analisar a eficiência do sistema é necessário selecionar algumas métricas que representem a percepção de clientes e também a adequação das dimensões das centrais ao seu processo de atendimento e chegada. Essas métricas devem permitir a avaliação da freqüência e do comportamento de clientes que são atendidos e que abandonam o sistema. Também devem incorporar informações sobre as filas e a ocupação do sistema.

As chamadas ocorridas no instante que o sistema está completo, ou seja, $s$ clientes sendo atendidos/servidos e $r$ clientes aguardando na fila; não serão consideradas nas medidas de desempenho.

No regime estacionário, as medidas que serão utilizadas neste trabalho são apresentadas a seguir:

(1) $P(W=0)$ : a probabilidade de um cliente, que entra no sistema, não ter que esperar pelo início do serviço;

(2) $P(A)$ : a probabilidade de um cliente, que entra no sistema, abandonar;

(3) $E[Q]$ : a média do tamanho da fila em um tempo arbitrário;

(4) $\operatorname{Var}(Q)$ : a variância do tamanho da fila em um tempo arbitrário; 
(5) $E[N]$ : o valor esperado do número de clientes no sistema em um tempo arbitrário;

(6) $E[W \mid S]$ : média do tempo de espera de um cliente na fila, dado que ele é servido;

(7) $\operatorname{Var}(W \mid S)$ : variância do tempo de espera de um cliente na fila, dado que ele é servido;

(8) $E[W \mid A]$ : média do tempo de espera de um cliente, dado que ele abandona;

(9) $\operatorname{Var}(W \mid A)$ : variância do tempo de espera de um cliente, dado que ele abandona;

(10) $P(W \leqslant t \mid S)$ : a probabilidade de que o tempo de espera seja menor que determinado valor $t$, dado que o cliente é servido;

(11) $P(W \leqslant t \mid A)$ : a probabilidade de que o tempo de espera seja menor que determinado valor $t$, dado que o cliente abandona.

As medidas (1) e (2), que são $P(W=0)$ e $P(A)$ respectivamente, permitem avaliar o dimensionamento de centrais de atendimentos e também estudar o comportamento (da paciência) dos clientes na fila. Por exemplo, caso $P(W=0)$ seja elevada e $P(A)$ seja pequena teríamos indícios de que talvez a central de atendimento esteja super dimensionada. Também, caso $P(W=0)$ seja pequena e $P(A)$ grande, é possível que a central de atendimento esteja sub-dimensionada.

O tamanho médio da fila, a sua variância e o número esperado de clientes no sistema, são respectivamente as medidas (3), (4) e (5) $(E[Q], \operatorname{Var}(Q)$ e $E[N])$ e também fornecem indicações sobre o dimensionamento de centrais de atendimento. Por exemplo, caso $E[Q]$ seja relativamente grande (com relação a $s$ ) e $\operatorname{Var}(Q)$ pequena tem-se indicação que as filas são freqüentes e tendem a perdurar durante todo o período de atendimento. Caso $\operatorname{Var}(Q)$ seja elevada e $E[Q]$ relativamente pequena espera-se observar filas com menor freqüência, no entanto, elas tendem a ser grandes quando se formarem.

As medidas (6) e (7), que são $E[W \mid S]$ e $\operatorname{Var}(W \mid S)$ respectivamente, permitem estudar o comportamento de clientes que são servidos pelo sistema e as medidas (8) e (9), que são $E[W \mid A] \mathrm{e}$ $\operatorname{Var}(W \mid A)$ respectivamente, indica o comportamento daqueles que abandonaram o sistema. Elas fornecem o tempo médio que clientes têm esperado na fila antes de abandonar ou o tempo que eles têm esperado na fila antes de serem servidos e, em termos estratégicos, podem ser utilizadas para definir metas ou comparar sistemas. Do ponto de vista dos clientes a principal medida de desempenho é o tempo de espera no sistema, antes do atendimento ou do abandono, que neste trabalho é representado por $E[W]$. 
Pode-se utilizar as medidas (10) e (11) para realizar análise de sensibilidade do sistema ou definir metas. Uma regra, frequentemente usada, em centrais de atendimento é a $80-20$ (ver Chevalier, Shumsky e Tabordon em [4]), definindo que a probabilidade de que o tempo de espera seja menor ou igual a $20 \%$ do tempo médio de serviço, deve ser maior ou igual a $80 \%$.

\subsection{Aproximação Markoviana para Abandonos}

A aproximação proposta por Whitt em [15] é baseada na construção de taxas de abandonos que dependem de duas variáveis: a posição do cliente na fila e o número de clientes na fila.

Diferentemente de modelos de filas com um único servidor, modelos de filas com um grande número de servidores tendem a ter um tempo médio de espera pequeno, comparado com o tempo médio de atendimento dos clientes.

Ao definir o tempo médio de espera de um cliente na fila, dado que o sistema possui abandono, uma componente importante é a taxa de abandono destes clientes, ver [6]. A função taxa de falha $h(t)$, que descreve a dinâmica da (im)paciência em $t$, é a propenção de abandono no instante $t$ condicionado a que o cliente estava na fila no instante anterior a $t$. Ela é definida pela probabilidade do cliente abandonar, dado que não abandonou até o tempo $t$.

Assim

$$
h(t)=\frac{f(t)}{F^{c}(t)}, \quad t \geq 0
$$

em que $f(t)$ e $F^{c}(t)$ são respectivamente, a função densidade de probabilidade e a função de sobrevivência. Observe que $F^{c}(t)=1-F(t)$, onde $F(t)$ é a função de distribuição acumulada do tempo de abandono.

A idéia da aproximação é desenvolver uma taxa de falha para o abandono em função da posição do cliente na fila e do tamanho da fila. Assim, Whitt propõe calcular quanto tempo o cliente já esteve esperando na fila e considera a evolução do sistema que se inicia no estado $s+r$ ( $s$ clientes em serviço e $r$ clientes aguardando na fila), ignorando chegadas futuras.

Com este objetivo, os clientes contados a partir do fim da fila, ou seja, o último cliente aguardando na fila recebe o número um, o penúltimo cliente o número dois, e assim por diante, até o primeiro cliente aguardando na fila que recebe o número $r$. Uma ilustração pode ser observada na Figura 2.1. Nesta figura estamos considerando uma fila com $r$ clientes, isto é, o sistema tem $s+r$ clientes.

Para definir o tempo médio que o j-ésimo cliente está no sistema, assumimos um sistema com 


Clientes aguardando atendimento
\begin{tabular}{|c|c|c|c|c|c|c|c|c|}
\hline 1 & $2 \circ$ & $\ldots$ & (j-1)은 & jo & $(j+1) \circ$ & $\ldots$ & $(r-1) \circ$ & ro
\end{tabular}$\rightarrow$\begin{tabular}{c}
$s$ \\
Servidores \\
\hline
\end{tabular}

Figura 2.1: Contagem de clientes.

$s$ clientes sendo servidos e $k$ clientes aguardando na fila, sendo $1 \leqslant j \leqslant k$. Além disso, assumimos que após a chegada do $j$-ésimo cliente não ocorreram abandonos, assim ocorrem $j-1$ chegadas e estamos no meio do intervalo da próxima chegada, que será considerado de modo aproximado como um intervalo completo. Por este motivo, o $j$-ésimo cliente aguardou um intervalo de tempo de $j$ chegadas, após a sua chegada ao sistema.

Como a chegada de cada cliente é definida por uma distribuição de Poisson e sua taxa é $\lambda$, temos que o intervalo entre as chegadas possui uma distribuição Exponencial com média $\frac{1}{\lambda}$ e o tempo que o $j$-ésimo cliente está no sistema seria aproximadamente $j \frac{1}{\lambda}$.

Através da função taxa de falha definida por 2.1 e o tempo médio que o j-ésimo cliente está no sistema, Whitt em [15] propõe a seguinte definição para a taxa de abandono:

$$
\alpha_{j} \equiv h(j / \lambda), \quad 1 \leqslant j \leqslant k
$$

onde $\lambda$ é a média entre chegadas e $h$ é a função taxa de falha do tempo de abandono dada por (2.1). A taxa total de abandonos da fila seria

$$
\delta_{k} \equiv \sum_{j=1}^{k} \alpha_{j}=\sum_{j=1}^{k} h(j / \lambda) ; \quad \delta_{0}=0 .
$$

Assume-se que a função densidade de probabilidade acumulada $F$ do tempo de abandono possui densidade (caso não possua, Whitt em [15], propõe uma alternativa).

A aproximação proposta considera que a distribuição de abandono será Exponencial com parâmetro $\delta_{k}$, quando existirem $k$ clientes no sistema. 
Para ilustrar que a aproximação Markoviana é eficaz para o modelo $M / M / s / r+G$, alguns resultados numéricos são apresentados.

\begin{tabular}{|c|c|c|c|c|c|}
\hline \multirow{2}{*}{$\begin{array}{c}\text { Medidas } \\
\text { de } \\
\text { Desenpenho }\end{array}$} & \multicolumn{2}{|c|}{$\mathrm{M} / \mathrm{M} / 100 / 200+\mathrm{E}_{2}$} & \multicolumn{2}{|c|}{$\mathrm{M} / \mathrm{M} / 100 / 200+\mathrm{LN}(1,1)$} & \multirow{2}{*}{ 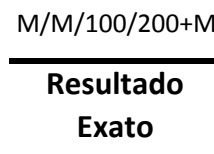 } \\
\hline & Simul. & Aprox. & Simul. & Aprox. & \\
\hline \multirow[t]{2}{*}{$P(W=0)$} & 0,246 & 0,25 & 0,242 & 0,247 & 0,408 \\
\hline & \pm 0.0020 & - & \pm 0.0026 & - & - \\
\hline \multirow[t]{2}{*}{$P(A)$} & 0,0378 & 0,0381 & 0,0376 & 0,0379 & 0,0499 \\
\hline & \pm 0.00032 & - & \pm 0.00032 & - & - \\
\hline \multirow[t]{2}{*}{$E[Q]$} & 11,75 & 11,41 & 11,42 & 11,02 & 5,09 \\
\hline & \pm 0.075 & - & \pm 0.071 & - & - \\
\hline \multirow[t]{2}{*}{$\operatorname{Var}(\mathrm{Q})$} & 129,2 & 121,9 & 115,6 & 107,2 & 44,6 \\
\hline & \pm 0.94 & - & \pm 0.46 & - & - \\
\hline \multirow[t]{2}{*}{$E[N]$} & 109,9 & 109,5 & 109,6 & 109,1 & 102 \\
\hline & \pm 0.091 & - & \pm 0.092 & - & - \\
\hline \multirow[t]{2}{*}{$E[W \mid S]$} & 0,1133 & 0,1102 & 0,1094 & 0,1058 & 0,049 \\
\hline & \pm 0.00072 & - & \pm 0.00067 & - & - \\
\hline \multirow[t]{2}{*}{$\operatorname{Var}(\mathrm{W} \mid \mathrm{S})$} & 0,0119 & 0,0113 & 0,0104 & 0,0097 & 0,0042 \\
\hline & \pm 0.000083 & - & \pm 0.000042 & - & - \\
\hline \multirow[t]{2}{*}{$E[W \mid A]$} & 0,1628 & 0,1521 & 0,1788 & 0,1642 & 0,0666 \\
\hline & \pm 0.00063 & - & \pm 0.00026 & - & - \\
\hline \multirow[t]{2}{*}{$\operatorname{Var}(\mathrm{W} \mid \mathrm{A})$} & 0,0079 & 0,0076 & 0,0054 & 0,0054 & 0,0031 \\
\hline & \pm 0.000061 & - & \pm 0.000024 & - & - \\
\hline \multirow[t]{2}{*}{$\mathrm{P}(\mathrm{W} \leq 0.1 \mid \mathrm{S})$} & 0,52 & 0,528 & 0,518 & 0,527 & 0,799 \\
\hline & \pm 0.0026 & - & \pm 0.0028 & - & - \\
\hline \multirow[t]{2}{*}{$P(W \leq 0.1 \mid A)$} & 0,273 & 0,316 & 0,14 & 0,204 & 0,767 \\
\hline & \pm 0.0019 & - & \pm 0.00064 & - & - \\
\hline \multirow[t]{2}{*}{$\mathrm{P}(\mathrm{W} \leq 0.2 \mid \mathrm{S})$} & 0,775 & 0,786 & 0,792 & 0,807 & 0,964 \\
\hline & \pm 0.0023 & - & \pm 0.0018 & - & - \\
\hline \multirow[t]{2}{*}{$P(W \leq 0.2 \mid A)$} & 0,688 & 0,726 & 0,644 & 0,706 & 0,97 \\
\hline & \pm 0.0027 & - & \pm 0.00066 & - & - \\
\hline $\begin{array}{l}\text { Observação: os m } \\
\text { distribuição de ab }\end{array}$ & odelos possuem & n, respecti & $\begin{array}{l}\text { nte, } \\
\text { I-LN }(1,1)\end{array}$ & & \\
\hline
\end{tabular}

Tabela 2.1: Comparação das simulações em $M / M / r / s+G$.

Na Tabela 2.1, são mostrados resultados aproximados e simulados de Whitt para o modelo $M / M / 100 / 200+G$ com tempos de abandonos Erlang e Lognormal, média de chegadas $\lambda$ igual a 102 e tempo médio de abandono igual a 1. Também são apresentados os resultados numéricos exatos para o modelo $M / M / 100 / 200+M$. Note que neste caso a aproximação coincide com o resultado exato que pode ser calculado analiticamente. 
Assim, conforme Whitt em [15], pode ser observado que a aproximação proposta considerando a posição do cliente na fila, resulta em uma boa aproximação Markoviana para abandonos, substituindo o modelo original $M / M / s / r+G$ pelo modelo $M / M / s / r+M(n)$, onde $M(n)$ representa abandono Markoviano dependente do estado do sistema.

\subsection{Aproximação Markoviana para Tempos de Atendimento}

Se a distribuição do tempo de serviço não é Exponencial, o modelo $M / G / s / r+M(n)$ é ainda muito difícil de ser analisado analiticamente, desta forma aproximações adicionais são necessárias.

Dessa forma, Whitt em [15] propõe uma segunda aproximação no modelo, produzindo um modelo Markoviano que pode ser analisado de maneira mais fácil. Ele considera o tempo de atendimento aproximado para uma distribuição Exponencial com a mesma média.

A base da aproximação é a propriedade de insensibilidade, que ocorre quando o desempenho da distribuição estacionária de uma fila depende apenas da média de atendimento e não da particular distribuição escolhida. O modelo de perda Erlang (também conhecido como o modelo Erlang$B$ ), representado por $M / G / s / 0$, tem essa propriedade. O modelo $M / G / \infty$ também satisfaz a propriedade da insensibilidade. Note que o modelo $M / G / s / r+G$, sob carga leve, se comporta conforme o modelo $M / G / \infty$. Desta forma, o modelo $M / G / s / r+G$, sob carga leve, também satisfaz a propriedade da insensibilidade.

Por outro lado, para o modelo $M / M / s / \infty$ (também conhecido como modelo Erlang- $C$ ) ou o modelo intermediário com sala de espera finita, $M / M / s / r$, a propriedade da insensibilidade não é válida. Entretanto, a dependência da distribuição do tempo de serviço é muito menor para modelos com vários servidores, conforme avaliação de Mandelbaum e Schwartz em [11].

Assumindo que abandonos estão ocorrendo com uma taxa alta, o modelo $M / G / s / r+G$ se torna mais parecido com o modelo $M / G / s / 0$ (que possui a propriedade de insensibilidade) do que com o modelo $M / G / s / \infty$. Assim, quando há um nível razoável de abandono, o modelo $M / M / s / r+G$ pode ser uma boa aproximação para o modelo $M / G / s / r+G$.

As considerações acima permitem definir o contexto no qual as aproximações do modelo $M / M / s / r+G$ pelo modelo totalmente Markoviano, $M / M / s / r+M(n)$, são razoáveis.

Na Tabela 2.2 apresentamos as medidas de desempenho obtidas por simulações do modelo de fila $M / G / s / r+E_{2}$, com quatro distribuições de serviço diferentes com mesma média igual a 1. Para a distribuição de abandono usamos a mesma média de atendimento para os modelos de fila apresentados. Como esta segunda aproximação depende apenas da média do atendimento e não de sua distribuição, o resultado aproximado apresentado na última coluna é o mesmo para os 
modelos $M / D / s / r+E_{2}, M / E_{2} / s / r+E_{2}, M / M / s / r+E_{2}$ e $M / L N(1,1) / s / r+E_{2}$ (diferentemente da simulação que para cada modelo obtemos um resultado diferente). Observa-se na Tabela 2.2 que o resultado simulado está bastante próximo do resultado aproximado, indicando uma boa aproximação para as medidas estudadas.

\begin{tabular}{|c|c|c|c|c|c|}
\hline \multirow{2}{*}{$\begin{array}{c}\text { Medidas } \\
\text { de } \\
\text { Desenpenho }\end{array}$} & \multicolumn{4}{|c|}{ Distribuição do Serviço } & \multirow[b]{2}{*}{$M / M / 100 / 200+E_{2}$} \\
\hline & $M / D / 100 / 200+E_{2}$ & $\mathrm{M} / \mathrm{E} 2 / 100 / 200+\mathrm{E}_{2}$ & $\mathrm{M} / \mathrm{M} / 100 / 200+\mathrm{E}_{2}$ & $M / L N(1,1) / 100 / 200+E_{2}$ & \\
\hline$P(W=0)$ & $\begin{array}{c}0,18 \\
\pm 0.0013\end{array}$ & $\begin{array}{c}0,217 \\
\pm 0.0021\end{array}$ & $\begin{array}{c}0,246 \\
\pm 0.0020\end{array}$ & $\begin{array}{c}0,233 \\
\pm 0.0021\end{array}$ & $\begin{array}{c}0,25 \\
-\end{array}$ \\
\hline$P(A)$ & $\begin{array}{c}0,0309 \\
\pm 0.00017\end{array}$ & $\begin{array}{c}0,0351 \\
\pm 0.00029\end{array}$ & $\begin{array}{c}0,0378 \\
\pm 0.00032\end{array}$ & $\begin{array}{c}0,037 \\
\pm 0.00027\end{array}$ & $\begin{array}{c}0,0381 \\
-\end{array}$ \\
\hline$E[Q]$ & $\begin{array}{c}11,08 \\
\pm 0.042\end{array}$ & $\begin{array}{c}11,52 \\
\pm 0.075\end{array}$ & $\begin{array}{c}11,75 \\
\pm 0.075\end{array}$ & $\begin{array}{c}11,74 \\
\pm 0.063\end{array}$ & $\begin{array}{c}11,41 \\
-\end{array}$ \\
\hline $\operatorname{Var}(\mathrm{Q})$ & $\begin{array}{c}89,3 \\
\pm 0.40\end{array}$ & $\begin{array}{c}112 \\
\pm 0.71\end{array}$ & $\begin{array}{l}129,2 \\
\pm 0.94\end{array}$ & $\begin{array}{l}123,3 \\
\pm 0.72\end{array}$ & $\begin{array}{c}121,9 \\
-\end{array}$ \\
\hline$E[N]$ & $\begin{array}{c}109,9 \\
\pm 0.049\end{array}$ & $\begin{array}{c}109,9 \\
\pm 0.092\end{array}$ & $\begin{array}{c}109,9 \\
\pm 0.091\end{array}$ & $\begin{array}{c}110 \\
\pm 0.72\end{array}$ & $\begin{array}{c}109,5 \\
-\end{array}$ \\
\hline$E[W \mid S]$ & $\begin{array}{c}0,1078 \\
\pm 0.00038\end{array}$ & $\begin{array}{c}0,1115 \\
\pm 0.00071\end{array}$ & $\begin{array}{c}0,1133 \\
\pm 0.00072\end{array}$ & $\begin{array}{c}0,1133 \\
\pm 0.00061\end{array}$ & $\begin{array}{c}0,1102 \\
-\end{array}$ \\
\hline $\operatorname{Var}(\mathrm{W} \mid \mathrm{S})$ & $\begin{array}{c}0,0079 \\
\pm 0.000032\end{array}$ & $\begin{array}{c}0,0101 \\
\pm 0.000061\end{array}$ & $\begin{array}{c}0,0119 \\
\pm 0.000083\end{array}$ & $\begin{array}{c}0,0113 \\
\pm 0.000061\end{array}$ & $\begin{array}{c}0,0113 \\
-\end{array}$ \\
\hline$E[W \mid A]$ & $\begin{array}{c}0,1343 \\
\pm 0.00028\end{array}$ & $\begin{array}{c}0,1508 \\
\pm 0.00042\end{array}$ & $\begin{array}{c}0,1628 \\
\pm 0.00063\end{array}$ & $\begin{array}{c}0,1589 \\
\pm 0.00039\end{array}$ & $\begin{array}{c}0,1521 \\
-\end{array}$ \\
\hline $\operatorname{Var}(\mathrm{W} \mid \mathrm{A})$ & $\begin{array}{c}0,0051 \\
\pm 0.000028\end{array}$ & $\begin{array}{c}0,0067 \\
\pm 0.000044\end{array}$ & $\begin{array}{c}0,0079 \\
\pm 0.000061\end{array}$ & $\begin{array}{c}0,0075 \\
\pm 0.000047\end{array}$ & $\begin{array}{c}0,0076 \\
-\end{array}$ \\
\hline$P(W \leq 0.1 \mid S)$ & $\begin{array}{c}0,501 \\
\pm 0.0018\end{array}$ & $\begin{array}{c}0,51 \\
\pm 0.0030\end{array}$ & $\begin{array}{c}0,52 \\
\pm 0.0026\end{array}$ & $\begin{array}{c}0,514 \\
\pm 0.0025\end{array}$ & $\begin{array}{c}0,528 \\
-\end{array}$ \\
\hline$P(W \leq 0.1 \mid A)$ & $\begin{array}{c}0,358 \\
\pm 0.0014\end{array}$ & $\begin{array}{c}0,305 \\
\pm 0.0014\end{array}$ & $\begin{array}{c}0,273 \\
\pm 0.0019\end{array}$ & $\begin{array}{c}0,283 \\
\pm 0.00088\end{array}$ & $\begin{array}{c}0,316 \\
-\end{array}$ \\
\hline$P(W \leq 0.2 \mid S)$ & $\begin{array}{c}0,833 \\
\pm 0.0013\end{array}$ & $\begin{array}{c}0,795 \\
\pm 0.0023\end{array}$ & $\begin{array}{c}0,775 \\
\pm 0.0023\end{array}$ & $\begin{array}{c}0,78 \\
\pm 0.0020\end{array}$ & $\begin{array}{c}0,786 \\
-\end{array}$ \\
\hline$P(W \leq 0.2 \mid A)$ & $\begin{array}{c}0,818 \\
\pm 0.0013\end{array}$ & $\begin{array}{c}0,74 \\
0,0019\end{array}$ & $\begin{array}{c}0,688 \\
\pm 0.0027\end{array}$ & $\begin{array}{c}0,705 \\
\pm 0.0018\end{array}$ & $\begin{array}{c}0,726 \\
-\end{array}$ \\
\hline \multicolumn{6}{|c|}{$\begin{array}{l}\text { Observação: } \\
\text { - Os modelos possuem mesma taxa de chegadas } \lambda=102 \\
\text { mesma distribuição de abandono } E_{2} \text {. } \\
\text { - A distribução de serviço varia para } \mathrm{E} 2 \text { com SCV }=0.5, \mathrm{M} \text { com SCV }=1.0, \mathrm{LN}(1,1) \text { com SCV }=1.0 \text { e } \\
\mathrm{LN}(1,4) \text { com SCV }=4.0 \text {. }\end{array}$} \\
\hline
\end{tabular}

Tabela 2.2: Comparação das simulações em $M / G / s / r+E_{2}$. 


\subsection{Distribuição Estacionária do Modelo Markoviano}

\subsubsection{Distribuição Estacionária do Processo de Nascimento e Morte}

Seja $N(t), t \geq 0$, o número de clientes no sistema $M / M / r / s+M(n)$ no tempo $t$. Esse processo pode ser descrito por um processo de nascimento e morte, com uma taxa de chegada (ou taxa de nascimento) $\lambda$ e taxa de saída (ou taxa de morte) formada a partir da soma da taxa total de atendimento e da taxa total de abandono.

Para um determinado estado do modelo, com $k$ clientes no sistema, a taxa de morte é dada por

$$
\mu_{k}= \begin{cases}k \mu, & 1 \leqslant k \leqslant s \\ s \mu+\delta_{k-s}, & s+1 \leqslant k \leqslant s+r\end{cases}
$$

onde $\mu$ é a taxa de atendimento individual e $\delta_{k}$ é a taxa total de abandono quando há $k$ clientes esperando na fila (obtido através de (2.3) em aproximação da $M / G / r / s+G$ ).

Desta maneira, a probabilidade de que haja $k$ pessoas no sistema após o processo atingir a estacionaridade é dada por:

$$
p_{k} \equiv P(N=k) \equiv \lim _{t \longrightarrow \infty} P(N(t)=k \mid N(0)=i)
$$

onde $i$ é o número de clientes no sistema no instante $0,1 \leqslant i \leqslant k$.

Esses $p_{k}$ devem ser calculados de maneira a garantir a validade das equações de balanço local

$$
p_{k} \lambda=p_{k+1} \mu_{k+1}, \quad 0 \leqslant k \leqslant s+r-1 .
$$

Assumindo que o número de servidores do sistema tenha sido escolhido de uma maneira razoável, espera-se que $p_{s}$ seja a maior probabilidade do estado estacionário do sistema. Assim, por conveniência, pode-se calcular cada uma dessas probabilidades de forma recursiva a partir de $p_{s}$. Assumindo $x_{s}=1$, com base na equação de balanço local pode-se escrever

$$
x_{s+k+1}=\frac{\lambda x_{s+k}}{\mu_{s+k+1}}, \quad 0 \leqslant k \leqslant r-1
$$

$\mathrm{e}$

$$
x_{k-1}=\frac{\mu_{k} x_{k}}{\lambda}, \quad 1 \leqslant k \leqslant s .
$$

E os $p_{k}$ podem ser obtidos através da normalização dos $x_{k}$, ou seja,

$$
y=\sum_{k=0}^{s+r} x_{k}
$$


Então,

$$
p_{k}=x_{k} / y, \quad 0 \leqslant k \leqslant s+r .
$$

\subsubsection{Probabilidade de um Cliente ser Atendido ou Abandonar}

O processo de chegada é um processo de Poisson e pela propriedade PASTA (Poisson Arrivals See Time Averages), a distribuição do número de clientes no sistema em tempo contínuo é igual à distribuição vista pelos clientes ao chegarem no sistema.

A probabilidade de uma chamada ser bloqueada ou recusada (não entrar no sistema) é a probabilidade do cliente encontrar $(s+r)$ clientes no sistema. Assim, considerando apenas as entradas

no sistema, temos que a probabilidade de um cliente entrar no sistema e encontrar $k$ outros clientes (aguardando atendimento ou sendo atendidos) é dada por

$$
p_{k}^{a}=\frac{p_{k}}{(1-P(\text { Recusa }))}=\frac{p_{k}}{1-p_{s+r}} ;
$$

em que $p_{k}$ foi calculado em 2.10 .

A probabilidade do cliente entrar no sistema e ser atendido imediatamente, ou seja, ter pelo menos um servidor desocupado é dada pela soma das probabilidades do sistema conter de 0 até $(s-1)$ clientes. Assim

$$
P(\text { Atendimento Imediato }) \equiv P(W=0)=\sum_{k=0}^{s-1} p_{k}^{a}
$$

Considere, agora, a situação de um cliente entrar no sistema e ser servido. Para que isto ocorra é necessário que ou o cliente receba atendimento imediato, ou o cliente entre no sistema e não abandone até o início de seu atendimento. A seguir definimos algumas equações para nos auxiliar no cálculo desta probabilidade.

Para desenvolver as expressões, consideramos o regime estacionário e um cliente típico que entra no sistema com todos os serviddores ocupados e vai para a fila, que passa a ter $k$ clientes. As posições dos clientes são numeradas de trás para frente, isto é, o cliente típico está inicialmente na posição 1 e o primeiro da fila na posição $k$. A partir daí o sistema evolui desconsiderando novas chegadas e esse cliente típico pode abandonar ou permanecer até ser atendido.

Seja $\gamma_{k, j}$ a probabilidade do cliente típico, que foi o $k$-ésimo a chegar, abandonar o sistema na posição $j$ (contada do fim da fila). 
Então, $\gamma_{k, j}$ pode ser definido aproximadamente por

$$
\gamma_{k, j} \approx \frac{\alpha_{j}}{s \mu+\left(\delta_{k}-\delta_{j-1}\right)}
$$

para $1 \leqslant j \leqslant k$, com $\delta_{0} \equiv 0$ e $\alpha_{j}$ definido em 2.2 .

Seja $m_{k, j}$ a média de tempo entre o $(j-1)$-ésimo e j-ésimo eventos de partida, que podem ocorrer por abandono ou por fim de serviço. Então $m_{k, j}$ é definido, aproximadamente, por

$$
m_{k, j} \approx \frac{1}{s \mu+\left(\delta_{k}-\delta_{j-1}\right)}
$$

para $1 \leqslant j \leqslant k, \operatorname{com} \delta_{0} \equiv 0$.

Note que para o modelo $M / M / s / r+M$, no qual $\alpha_{j}=\alpha$ para todo $j$, estas aproximações são exatas. Desta forma, teríamos

$$
\gamma_{k, j}=\frac{\alpha}{s \mu+(k-j+1) \alpha} \quad \text { e } \quad m_{k, j}=\frac{1}{s \mu+(k-j+1) \alpha}
$$

$\operatorname{com} \delta_{0} \equiv 0$ e $\delta_{k} \equiv \sum_{j=1}^{k} \alpha_{j}$ definido em 2.3.

Para uma explicação intuitiva da equação 2.13, deve ser observado que após a chegada do $k$ ésimo cliente, assumimos que futuras chegadas são rejeitadas e as taxas de abandono dos clientes na fila são conhecidas, ou seja, o $k$-ésimo cliente possui taxa de abandono $\alpha_{k} \equiv h(k / \lambda)$. Para estudar o estado após $j-1$ saídas, é necessário observar a dinânica do sistema no tempo. Assim, como estamos considerando o tempo, deve ser considerado que os clientes que já estão na fila, aumentam seus tempos de espera a cada instante. Então passa ser necessário que a taxa de paciência destes clientes sejam alteradas conforme o tempo que o cliente já esperou e uma aproximação adicional é feita. Assumimos $1 / \lambda$ como o tempo entre cada intervalo de saída, desta forma, após $j-1$ partidas, se o $k$-ésimo cliente ainda estiver na fila, sua taxa de paciência passa a ser $\alpha_{k+(j-1)} \simeq h((k+(j-1)) / \lambda)$.

Com a aproximação acima resolvemos o problema de dependência da taxa de paciência ao tempo e obtemos a evolução da taxa de paciência. Contudo, ocorrendo $j-1$ saídas, a evolução do sistema depende se ocorreram saídas por fim de serviço ou por abandono. Desta forma, mais uma çãoaproximação é feita e assumimos que todas as $j-1$ saídas foram por fim de serviço. Assim, os primeiros $j-1$ clientes aguardando na fila, inicialmente com taxas de abandono $\left(\alpha_{j-1}, \ldots, \alpha_{l}\right)$, finalizam serviço. Então os demais $k-(j-1)$ clientes, com taxas de abandono iniciais $\left(\alpha_{1}, \ldots, \alpha_{k-(j-1)}\right)$, têm suas posições acrescidas de $j-1$, passam a ter taxas de paciência $\left(\alpha_{j}, \ldots, \alpha_{l}\right)$.

Dada a aproximação (2.13), calculamos a probabilidade do cliente típico entrar na fila e receber 
serviço.

$$
\Gamma_{k}=\left(1-\gamma_{k, 1}\right)\left(1-\gamma_{k, 2}\right) \ldots\left(1-\gamma_{k, k}\right)
$$

para $\gamma_{k, j}$ em (2.13).

Note que, $\gamma_{k, 1}$ é a probabilidade do cliente típico entrar no sistema, abandonar a fila na posição 1 e, então, $1-\gamma_{l, 1}$ é a probabilidade desse cliente entrar no sistema, não abandonar a fila quando estiver na posição 1 (contada à partir do fim da fila). Desta forma, $\Gamma_{k}$ é a probabilidade do cliente típico entrar no sistema e não abandonar a fila até início de serviço.

Calculando aproximadamente a probabilidade de uma chegada completar serviço, temos

$$
P(S)=\left(\sum_{k=0}^{s-1} p_{k}^{a}\right)+\sum_{k=0}^{r-1} p_{s+k}^{a} \Gamma_{k+1}=P\left(S_{I}\right)+P\left(S_{T}\right)
$$

Com a primeira parte da soma sendo a probabilidade de um cliente entrar no sistema e ser atendido imediatamente $\left(P\left(S_{I}\right)\right)$ e a segunda parte, a probabilidade do cliente entrar no sistema, encontrar fila e aguardar até o início do atendimento $\left(P\left(S_{T}\right)\right)$.

Se um cliente entra no sistema, ele sairá após ser servido ou por abandono e por este motivo, podemos expressar a probabilidade estacionária de um cliente que entra no sistema e o abandona como

$$
P(A)=1-P(S) .
$$

\subsubsection{Tempo de Espera para Clientes que são Atendidos}

Temos $E[W ; S]=E\left[W 1_{S}\right]$, onde $1_{B}$ é a função indicadora do evento $B\left(1_{\omega}=1\right.$ se $\omega \in B$, e $1_{\omega}=0$, caso contrário).

Assumimos que sucessivos intervalos entre partidas são variáves aleatórias independentes exponenciais, com as médias dadas em (2.14) e, utilizando as propriedades da distribuição Exponencial, definimos os dois primeiros momentos do tempo médio de um cliente que é atendido por

$$
E[W ; S]=\sum_{k=0}^{r-1} p_{s+k}^{a} \Gamma_{k+1} \sum_{j=1}^{k+1} m_{k+1, j}
$$

$\mathrm{e}$

$$
E\left[W^{2} ; S\right]=\sum_{k=0}^{r-1} p_{s+k}^{a} \Gamma_{k+1}\left(V_{k+1}+M_{k+1}^{2}\right),
$$


em que

$$
V_{k+1} \equiv \sum_{j=1}^{k+1} m_{k+1, j}^{2}
$$

$\mathrm{e}$

$$
M_{k+1} \equiv \sum_{j=1}^{k+1} m_{k+1, j}
$$

Então, os primeiro e segundo momentos do tempo de espera condicional, dado que o cliente certamente completa o serviço são

$$
E(W \mid S)=\frac{E[W ; S]}{P(S)}
$$

$\mathrm{e}$

$$
E\left(W^{2} \mid S\right)=\frac{E\left[W^{2} ; S\right]}{P(S)}
$$

Assim, a variância condicional é

$$
\operatorname{Var}(W \mid S) \equiv E\left(W^{2} \mid S\right)-(E(W \mid S))^{2} .
$$

Determina-se a distribuição do tempo de espera através da sua transformada de Laplace. Para isto, seja $\widehat{\omega}_{s}(z) \equiv E\left[\exp ^{-z W} 1_{\{S, W>0\}}\right]$ a transformada de Laplace de $W$ para clientes que são servidos, mas não imediatamente. Através de (2.19), temos

$$
\begin{array}{r}
\widehat{\omega}_{s}(z) \equiv E\left[\exp ^{-z W} 1_{\{S, W>0\}}\right]=E\left[\exp ^{-z W} ; S\right]= \\
=\sum_{k=0}^{r-1} p_{s+k}^{a} \Gamma_{k+1} \widehat{e}_{k+1}(z),
\end{array}
$$

onde

$$
\widehat{e}_{k+1}(z) \equiv \prod_{j=1}^{k+1}\left(\frac{m_{k+1, j}^{-1}}{m_{k+1, j}^{-1}+z}\right)
$$

A função de distribuição acumulada do tempo de espera de um cliente que será servido aguardar um intervalo de tempo $t$ e ser atendido, $P(0<W \leqslant t ; S)$, é obtida para qualquer $t$, invertendo numericamente a transformada de Laplace de $\widehat{\omega}_{s}(z) / z$, utilizando o método da série de Fourier. 
A função de distribuição acumulada do tempo de espera condicional é

$$
P(W \leqslant t \mid S)=\frac{P(W=0)+P(0<W \leqslant t ; S)}{P(S)} .
$$

\subsubsection{Probabilidade de um Cliente Abandonar}

A probabilidade do cliente abandonar é a probabilidade do cliente entrar no sistema, multiplicada pela probabilidade dele abandonar em algum momento:

$$
P(A)=\sum_{k=0}^{r-1} p_{s+k}^{a}\left(1-\Gamma_{k+1}\right) .
$$

Seja $W_{k}$, o tempo médio que o k-ésimo cliente permanece na fila.

O tempo médio de um cliente abandonar, é definido pelo produto da probabilidade do cliente entrar no sistema num certo instante e o tempo médio que ele permanece no sistema sabendo que ele irá abandonar. Assim,

$$
E\left[W 1_{A}\right]=\sum_{k=0}^{r-1} p_{s+k}^{a} E\left[W_{k+1} 1_{A}\right]
$$

e, de modo análogo, para o segundo momento:

$$
E\left[W^{2} 1_{A}\right]=\sum_{k=0}^{r-1} p_{s+k}^{a} E\left[W_{k+1}^{2} 1_{A}\right] .
$$

Ainda, a esperança do tempo médio no sistema do k-ésimo cliente, sabendo que este o abandona, é a soma da taxa de abandono, multiplicada pelo tempo médio condicionado ao $k$-ésimo cliente abandonar, ou seja,

$$
\begin{gathered}
E\left[W_{k} 1_{A}\right]=\gamma_{k, 1} m_{k, 1}+\left(1-\gamma_{k, 1}\right) \gamma_{k, 2}\left(m_{k, 1}+m_{k, 2}\right)+\left(1-\gamma_{k, 1}\right)\left(1-\gamma_{k, 2}\right) \gamma_{k, 3} . \\
\cdot\left(m_{k, 1}+m_{k, 2}+m_{k, 3}\right)+\ldots+\left(1-\gamma_{k, 1}\right) \cdots\left(1-\gamma_{k, k-1}\right) \gamma_{k, k}\left(m_{k, 1}+\ldots+m_{k, k}\right) .
\end{gathered}
$$

De modo análogo temos:

$$
\begin{array}{r}
E\left[W_{k}^{2} 1_{A}\right]=\gamma_{k, 1} 2 m_{k, 1}^{2}+\left(1-\gamma_{k, 1}\right) \gamma_{k, 2}\left(m_{k, 1}^{2}+m_{k, 2}^{2}+\left(m_{k, 1}+m_{k, 2}\right)^{2}\right)+\ldots+\left(1-\gamma_{k, 1}\right) . \\
\cdot\left(1-\gamma_{k, 2}\right) \cdots\left(1-\gamma_{k, k-1}\right) \gamma_{k, k}\left(m_{k, 1}^{2}+\ldots+m_{k, k}^{2}+\left(m_{k, 1}+\ldots+m_{k, k}\right)^{2}\right) .
\end{array}
$$

Então, os momentos condicionais são

$$
E(W \mid A)=\frac{E\left[W 1_{A}\right]}{P(A)} \quad \text { e } \quad E\left(W^{2} \mid A\right)=\frac{E\left[W^{2} 1_{A}\right]}{P(A)}
$$


para $P(A)$ em $(2.29)$.

A variância condicional é dada por:

$$
\operatorname{Var}(W \mid A) \equiv E\left(W^{2} \mid A\right)-(E(W \mid A))^{2}
$$

Seja $\widehat{a}_{k}(z) \equiv E\left[{ }^{e-z W} 1_{A}\right]$ a transformada de Laplace de $W$ para clientes que entram no sistema e o abandonam. E com auxilio das equações (2.26) e (2.32). Vem,

$$
\widehat{a}(z)=\sum_{k=0}^{r-1} p_{s+k}^{a} \widehat{a}_{k+1}(z)
$$

em que

$$
\widehat{a}_{k}(z)=\gamma(k, 1)\left(\frac{m_{k, 1}^{-1}}{m_{k, 1}^{-1}+z}\right)+\sum_{j=2}^{k} \gamma_{k, j}\left(\frac{m_{k, j}^{-1}}{m_{k, j}^{-1}+z}\right) \cdot \prod_{\ell=1}^{j-1}\left[\left(1-\gamma_{k, \ell}\right)\left(\frac{m_{k, \ell}^{-1}}{m_{k, \ell}^{-1}+z}\right)\right] .
$$

Através de $P(0<W \leqslant t ; S)$ obtida anteriormente, podemos calcular $P(W ; A)$ utilizando a inversa numérica da transformada de Laplace de $\widehat{a}(z) / z$.

A função densidade de probabilidade condicional do tempo de abandono, dado que o cliente irá abandonar é

$$
P(W \leqslant t \mid A)=\frac{P(W \leqslant t ; A)}{P(A)} .
$$

Assim a distribuição do tempo de espera para todos os clientes, independentemente se eles abandonam ou são atendidos, é

$$
P(W \leqslant t)=P(W=0)+P(0<W \leqslant t ; S)+P(W \leqslant t ; A), \quad t>0 .
$$

Note que estamos somando todas as possibilidades de acontecimento quando um cliente entra no sistema, incluindo as situações em que o cliente não necessita esperar, o cliente aguarda um tempo $t$ e é atendido e o cliente aguarda um tempo $t$ e abandona o sistema. Esta soma nos dá a função distribuição de probabilidade do tempo de espera de um cliente que entra no sistema.

\subsection{Descrição do algoritmo e resultados numéricos}

Nesta seção vamos apresentar o algoritmo que desenvolvemos para as simulações e o algoritmo desenvolvido para calcular as aproximações propostas por Whitt, em [15]. Também apresentaremos os resultados numéricos, da aproximação e da simulação, fazendo uma comparação com os resultados apresentados por Whitt. Os algoritmos foram desenvolvidos utilizando o software R. 
O algoritmo para fazer a simulação está estruturado de forma iterativa de modo que, para cada momento onde ocorre um evento, atualiza-se o estado do sistema (cliente nos servidores, clientes na fila, tempo de espera dos clientes, etc). Ao final são calculadas as medidas de desempenho do sistema.

Whitt, em [15], utiliza uma Central de Atendimento mais próxima possível da realidade e, os parâmetros utilizados foram números grandes. A Central de Atendimento estudada por Whitt possui 100 atendentes, sala de espera de tamanho 200, taxa de abandono 1, taxa de serviço 1 e taxa de chegada 102. Além disso, em cada iteração feita por Whitt, foram considerados 5 milhões de clientes passando pelo sistema. Com a utilização destes parâmetros e este alto volume de chegadas, a simulação torna-se lenta. A solução encontrada foi utilizar 100 mil chegadas de clientes para cada iteração, mantendo todos os outros parâmetros.

Nas Tabelas 2.3 e 2.4, apresentamos a simulação dos modelos de fila $M / M / 100 / 200+M$ e $M / E_{2} / 100 / 200+E_{2}$, em que a chegada segue um Processo de Poisson com taxa 102, o atendimento é feito com distribuição Exponencial de taxa 1, temos 100 servidores, 200 espaços na sala de espera e o abandono ocorre com distribuição Exponencial de taxa 1.

Apresentamos nas mesmas Tabelas 2.3 e 2.4, a variação e o percentual de variação do Resultado Simulado (por Whitt) e do Resultado Simulado (pelo nosso algoritmo). Na aproximação, obtivemos resultados muito bons, onde o maior erro é de $5 \%$ na $\operatorname{Var}(W \mid S)$, com valor absoluto de 0,0006. Desta forma, validamos nosso algoritmo de simulação e de aproximação. Os resultados foram comparados com os dados obtidos por Whitt e, como foram muito próximos, assumimos que o algoritmo simula corretamente o modelo.

No algoritmo das aproximações, as medidas de desempenho foram desenvolvidas conforme descrito na Seção 2.4, e nossos resultados e os do Whitt são apresentados nas Tabelas 2.5 e 2.6. Na simulação apenas a $\operatorname{Var}(W \mid S)$ do modelo $M / E_{2} / 100 / 200+E_{2}$ possui uma variação percentual maior, mas com uma variação absoluta de 0,0061. Todas as demais medidas de desempenho estão abaixo de $2 \%$ de erro. Quanto a aproximação, no modelo $M / E_{2} / 100 / 200+E_{2}$, a $\operatorname{Var}(W \mid S)$ possui o maior percentual de erro, mas com um valor absoluto de 0,0006 e a $\operatorname{Var}(W \mid A)$ possui um percentual de erro de 3,8\%, mas com um valor absoluto de 0,0003. As demais medias estão com erro em torno de zero. 


\begin{tabular}{|c|c|c|c|c|}
\hline \multicolumn{5}{|c|}{ Simulação do modelo de fila $\mathrm{M} / \mathrm{M} / 100 / 200+\mathrm{M}$} \\
\hline $\begin{array}{l}\text { Medidas de } \\
\text { Desempenho }\end{array}$ & $\begin{array}{c}\text { Resultado } \\
\text { Simulado } \\
\text { (Whitt) }\end{array}$ & $\begin{array}{l}\text { Resultado } \\
\text { Simulado } \\
\text { (Gabriela) }\end{array}$ & $\begin{array}{c}\text { Variação } \\
\text { (W-G) }\end{array}$ & $\begin{array}{c}\% \text { de } \\
\text { Variação } \\
(\mathbf{W}-\mathrm{G}) / \mathrm{W}\end{array}$ \\
\hline$P(W=0)$ & 0,4092 & 0,4048 & $-0,0044$ & $-1,07 \%$ \\
\hline$P(A)$ & 0,0498 & 0,0505 & 0,0007 & $1,45 \%$ \\
\hline$E[Q]$ & 5,0730 & 5,0956 & 0,0226 & $0,45 \%$ \\
\hline $\operatorname{Var}(Q)$ & 44,4000 & 44,6498 & 0,2498 & $0,56 \%$ \\
\hline$E[N]$ & 102,0000 & 102,0255 & 0,0255 & $0,02 \%$ \\
\hline$E[W \mid S]$ & 0,0489 & 0,0492 & 0,0003 & $0,51 \%$ \\
\hline $\operatorname{Var}(\mathrm{W} \mid \mathrm{S})$ & 0,0042 & 0,0042 & 0,0000 & $0,00 \%$ \\
\hline$E[W \mid A]$ & 0,0665 & 0,0668 & 0,0003 & $0,47 \%$ \\
\hline $\operatorname{Var}(\mathrm{W} \mid \mathrm{A})$ & 0,0031 & 0,0032 & 0,0001 & $0,00 \%$ \\
\hline $\mathrm{P}(\mathrm{W} \leq 0.1 \mid \mathrm{S})$ & 0,7994 & 0,7988 & $-0,0006$ & $-0,08 \%$ \\
\hline$P(W \leq 0.1 \mid A)$ & 0,7678 & 0,7650 & $-0,0028$ & $-0,36 \%$ \\
\hline$P(W \leq 0.2 \mid S)$ & 0,9648 & 0,9644 & $-0,0004$ & $-0,04 \%$ \\
\hline$P(W \leq 0.2 \mid A)$ & 0,9705 & 0,9695 & $-0,0010$ & $-0,10 \%$ \\
\hline $\begin{array}{l}\text { Observação: Simulaç } \\
\text { satisfazendo a distrit } \\
\text { Exponencial(1), distr } \\
\text { de espera. }\end{array}$ & $\begin{array}{l}\text { modelo de fila } \\
\text { de Poisson con } \\
\text { do abandono }\end{array}$ & $\begin{array}{l}\text { M/100/200+M, } \\
\text { ka 102, distribui } \\
\text { onencial(1), } 100\end{array}$ & $\begin{array}{l}200 \text { mil che } \\
\text { do atendime } \\
\text { rvidores, } 200\end{array}$ & $\begin{array}{l}\text { las } \\
\text { paços na sala }\end{array}$ \\
\hline
\end{tabular}

Tabela 2.3: Comparação da simulação do modelo de fila $M / M / 100 / 200+M$. 


\begin{tabular}{|c|c|c|c|c|}
\hline \multicolumn{5}{|c|}{ Simulação do modelo de fila $M / E_{2} / 100 / 200+E_{2}$} \\
\hline $\begin{array}{l}\text { Medidas de } \\
\text { Desempenho }\end{array}$ & $\begin{array}{c}\text { Resultado } \\
\text { Simulado } \\
\text { (Whitt) }\end{array}$ & $\begin{array}{l}\text { Resultado } \\
\text { Simulado } \\
\text { (Gabriela) }\end{array}$ & $\begin{array}{c}\text { Variação } \\
\text { (W-G) }\end{array}$ & $\begin{array}{c}\% \text { de } \\
\text { Variação } \\
(W-G) / W\end{array}$ \\
\hline$P(W=0)$ & 0,2170 & 0,2208 & 0,0038 & $1,77 \%$ \\
\hline$P(A)$ & 0,0351 & 0,0351 & 0,0000 & $0,13 \%$ \\
\hline$E[Q]$ & 11,5200 & 11,4594 & $-0,0606$ & $-0,53 \%$ \\
\hline $\operatorname{Var}(\mathrm{Q})$ & 112,0000 & 113,8626 & 1,8626 & $1,66 \%$ \\
\hline$E[N]$ & 109,9000 & 109,8072 & $-0,0928$ & $-0,08 \%$ \\
\hline$E[W \mid S]$ & 0,1115 & 0,1112 & $-0,0003$ & $-0,31 \%$ \\
\hline $\operatorname{Var}(\mathrm{W} \mid \mathrm{S})$ & 0,0101 & 0,0040 & $-0,0061$ & $-60,51 \%$ \\
\hline$E[W \mid A]$ & 0,1508 & 0,1518 & 0,0010 & $0,65 \%$ \\
\hline $\operatorname{Var}(\mathrm{W} \mid \mathrm{A})$ & 0,0067 & 0,0068 & 0,0001 & $1,43 \%$ \\
\hline$P(W \leq 0.1 \mid S)$ & 0,5100 & 0,5147 & 0,0047 & $0,92 \%$ \\
\hline$P(W \leq 0.1 \mid A)$ & 0,3050 & 0,3037 & $-0,0013$ & $-0,44 \%$ \\
\hline$P(W \leq 0.2 \mid S)$ & 0,7950 & 0,7942 & $-0,0008$ & $-0,10 \%$ \\
\hline$P(W \leq 0.2 \mid A)$ & 0,7400 & 0,7359 & $-0,0041$ & $-0,55 \%$ \\
\hline $\begin{array}{l}\text { Observação: Simula } \\
\text { satisfazendo a distr } \\
\text { distribuição do aba }\end{array}$ & $\begin{array}{l}\text { lo modelo de } \mathrm{fi} \\
\text { Oo de Poisson c } \\
\text { Erlang2, } 100 \mathrm{~s}\end{array}$ & $\begin{array}{l}/ E_{2} / 100 / 200+E \\
\text { taxa } 102, \text { distrib } \\
\text { dores, } 200 \text { esp }\end{array}$ & $\begin{array}{l}\text { om } 200 \text { mil ch } \\
\text { ão do atendin } \\
\text { s na sala de es }\end{array}$ & $\begin{array}{l}\text { adas } \\
\text { nto Erlang2, } \\
\text { era. }\end{array}$ \\
\hline
\end{tabular}

Tabela 2.4: Comparação da simulação do modelo de fila $M / E_{2} / 100 / 200+E_{2}$. 


\begin{tabular}{|c|c|c|c|c|}
\hline \multicolumn{5}{|c|}{ Aproximação do modelo de fila $\mathrm{M} / \mathrm{M} / 100 / 200+\mathrm{M}$} \\
\hline $\begin{array}{c}\text { Medidas } \\
\text { de } \\
\text { Desempenho }\end{array}$ & $\begin{array}{c}\text { Resultado } \\
\text { Aproximado } \\
\text { (Whitt) }\end{array}$ & $\begin{array}{c}\text { Aproximação } \\
\text { (Gabriela) }\end{array}$ & $\begin{array}{c}\text { Variação } \\
\text { (W-G) }\end{array}$ & $\begin{array}{c}\text { \% de } \\
\text { Variação } \\
(\mathrm{W}-\mathrm{G}) / \mathrm{W}\end{array}$ \\
\hline$P(W=0)$ & 0,4083 & 0,4083 & 0,0000 & $0,00 \%$ \\
\hline$P(A)$ & 0,0499 & 0,0499 & 0,0000 & $0,00 \%$ \\
\hline$E[Q]$ & 5,0920 & 5,0916 & $-0,0004$ & $-0,01 \%$ \\
\hline $\operatorname{Var}(\mathrm{Q})$ & 44,6000 & 44,6138 & 0,0138 & $0,03 \%$ \\
\hline$E[N]$ & 102,0000 & 102,0000 & 0,0000 & $0,00 \%$ \\
\hline$E[W \mid S]$ & 0,0490 & 0,0490 & 0,0000 & $0,00 \%$ \\
\hline $\operatorname{Var}(\mathrm{W} \mid \mathrm{S})$ & 0,0042 & 0,0042 & 0,0000 & $0,00 \%$ \\
\hline$E[W \mid A]$ & 0,0666 & 0,0666 & 0,0000 & $0,00 \%$ \\
\hline $\operatorname{Var}(\mathrm{W} \mid \mathrm{A})$ & 0,0031 & 0,0031 & 0,0000 & $0,00 \%$ \\
\hline $\mathrm{P}(\mathrm{W} \leq 0.1 \mid \mathrm{S})$ & 0,7986 & 0,7986 & 0,0000 & $0,00 \%$ \\
\hline$P(W \leq 0.1 \mid A)$ & 0,7671 & 0,7671 & 0,0000 & $0,00 \%$ \\
\hline $\mathrm{P}(\mathrm{W} \leq 0.2 \mid \mathrm{S})$ & 0,9644 & 0,9644 & 0,0000 & $0,00 \%$ \\
\hline$P(W \leq 0.2 \mid A)$ & 0,9702 & 0,9702 & 0,0000 & $0,00 \%$ \\
\hline $\begin{array}{l}\text { Observação: Apro } \\
\text { distribuição de Po } \\
\text { do abandono Exp }\end{array}$ & $\begin{array}{l}\text { ação do modelo } \\
\text { n com taxa } 102 \text {, } \\
\text { ncial(1), } 100 \text { servi }\end{array}$ & $\begin{array}{l}\text { M/M/100/200+N } \\
\text { buição do atendim } \\
\text { s, } 200 \text { espaços na s }\end{array}$ & $\begin{array}{l}\text { m chegadas } \\
\text { Exponencial } \\
\text { de espera. }\end{array}$ & $\begin{array}{l}\text { sfazendo a } \\
\text { distribuição }\end{array}$ \\
\hline
\end{tabular}

Tabela 2.5: Comparação da Aproximação do modelo de fila $M / M / 100 / 200+M$. 


\begin{tabular}{|c|c|c|c|c|}
\hline \multicolumn{5}{|c|}{ Aproximação do modelo de fila $M / E_{2} / 100 / 200+E_{2}$} \\
\hline $\begin{array}{c}\text { Medidas } \\
\text { de } \\
\text { Desempenho }\end{array}$ & $\begin{array}{c}\text { Resultado } \\
\text { Aproximado } \\
\text { (Whitt) }\end{array}$ & $\begin{array}{c}\text { Aproximação } \\
\text { (Gabriela) }\end{array}$ & $\begin{array}{c}\text { Variação } \\
\text { (W-G) }\end{array}$ & $\begin{array}{c}\% \text { de } \\
\text { Variação } \\
(\mathrm{W}-\mathrm{G}) / \mathrm{W}\end{array}$ \\
\hline$P(W=0)$ & 0,2500 & 0,2495 & $-0,0005$ & $-0,20 \%$ \\
\hline$P(A)$ & 0,0381 & 0,0381 & 0,0000 & $0,00 \%$ \\
\hline$E[Q]$ & 11,4100 & 11,4056 & $-0,0044$ & $-0,04 \%$ \\
\hline $\operatorname{Var}(\mathrm{Q})$ & 121,9000 & 121,9129 & 0,0129 & $0,01 \%$ \\
\hline$E[N]$ & 109,5000 & 109,5164 & 0,0164 & $0,01 \%$ \\
\hline$E[W \mid S]$ & 0,1102 & 0,1102 & 0,0000 & $0,00 \%$ \\
\hline $\operatorname{Var}(\mathrm{W} \mid \mathrm{S})$ & 0,0119 & 0,0113 & $-0,0006$ & $-5,04 \%$ \\
\hline$E[W \mid A]$ & 0,1521 & 0,1521 & 0,0000 & $0,00 \%$ \\
\hline $\operatorname{Var}(W \mid A)$ & 0,0079 & 0,0076 & $-0,0003$ & $-3,80 \%$ \\
\hline$P(W \leq 0.1 \mid S)$ & 0,5280 & 0,5279 & $-0,0001$ & $-0,02 \%$ \\
\hline$P(W \leq 0.1 \mid A)$ & 0,3160 & 0,3159 & $-0,0001$ & $-0,03 \%$ \\
\hline$P(W \leq 0.2 \mid S)$ & 0,7860 & 0,7863 & 0,0003 & $0,04 \%$ \\
\hline$P(W \leq 0.2 \mid A)$ & 0,7260 & 0,7259 & $-0,0001$ & $-0,01 \%$ \\
\hline $\begin{array}{l}\text { Observação: Apro } \\
\text { distribuição de Po } \\
\text { abandono Erlang2 }\end{array}$ & $\begin{array}{l}\text { ação do modelo c } \\
\text { n com taxa } 102, \\
0 \text { servidores, } 200\end{array}$ & $\begin{array}{l}\text { a } M / E_{2} / 100 / 200+E \\
\text { buição do atendim } \\
\text { aços na sala de esp }\end{array}$ & $\begin{array}{l}m \text { chegadas } \\
\text { Erlang2, dis }\end{array}$ & $\begin{array}{l}\text { isfazendo a } \\
\text { uição do }\end{array}$ \\
\hline
\end{tabular}

Tabela 2.6: Comparação da Aproximação do modelo de fila $M / E_{2} / 100 / 200+E_{2}$. 


\section{Capítulo 3}

\section{Central de Atendimento com mais de um tipo de cliente.}

\subsection{Introdução}

Uma Central de Atendimento pode possuir clientes com comportamentos distintos, tendo em vista a grande variedade de serviços oferecidos. Além disso, em geral, os clientes possuem comportamentos distintos em situações parecidas, o que também pode gerar um modelo de filas com clientes de diferentes tipos.

Vamos considerar uma Central de Atendimento com $m$ ( $\operatorname{com} m \in \mathbb{N}-\{0\}$ ) tipos de clientes que compartilham a mesma fila e os mesmos servidores, mas possuem serviço e abandono diferentes. As chegadas são definidas por uma distribuição de Poisson com média $\lambda$, para qualquer tipo de cliente. O cliente é identificado após sua entrada no sistema e será do tipo $i$ com probabilidade $q_{i}$ em que $i \in\{1, \ldots, m\}$. O atendimento de cliente do tipo $i$ ocorrerá com distribuição Exponencial de taxa $\mu_{i}$ e os abandonos seguem distribuição $G_{i}$ para cliente tipo $i$, com $1 \leqslant i \leqslant m$. A Figura 3.1 ilustra o caso de uma central com $m$ tipos de clientes.

\subsection{Modelo $M / M^{i} / s / r+M^{i}(n)$}

Seguindo a mesma linha de Whitt em [15], vamos considerar a aproximação do modelo de fila $M / G^{i} / s / r+G^{i}$ pelo modelo $M / M^{i} / s / r+M^{i}(n)$, que possui distribuição Exponencial para o serviço e para o abandono.

Para $m \in \mathbb{N}-\{0\}$, seja $\mathcal{I}=\{1,2, \ldots, m\}$ o conjunto de possíveis tipos de clientes. Definimos por $e_{l}$ a situação na posição $l$ da fila, com $e_{l} \in \mathcal{I} \cup\{0\}$ e $l=\{1, \ldots, r\}$, sendo $r$ o número de espaços na sala de espera. Assim, $\widetilde{e}=\left(e_{1}, e_{2}, \ldots, e_{r}\right)$ descreve a fila indicando a sequência (em ordem de chegadas) de tipos de clientes e quando não houver cliente em alguma posição da fila esta posição será 0 , isto é,

$$
e_{l}= \begin{cases}0, & \text { se não há cliente na posição } l \\ i, & \text { se o cliente na posição } l \text { é do tipo } i\end{cases}
$$

Observe que se, para algum $l, e_{l}=0$, então $e_{l+1}=\ldots=e_{r}=0$. 


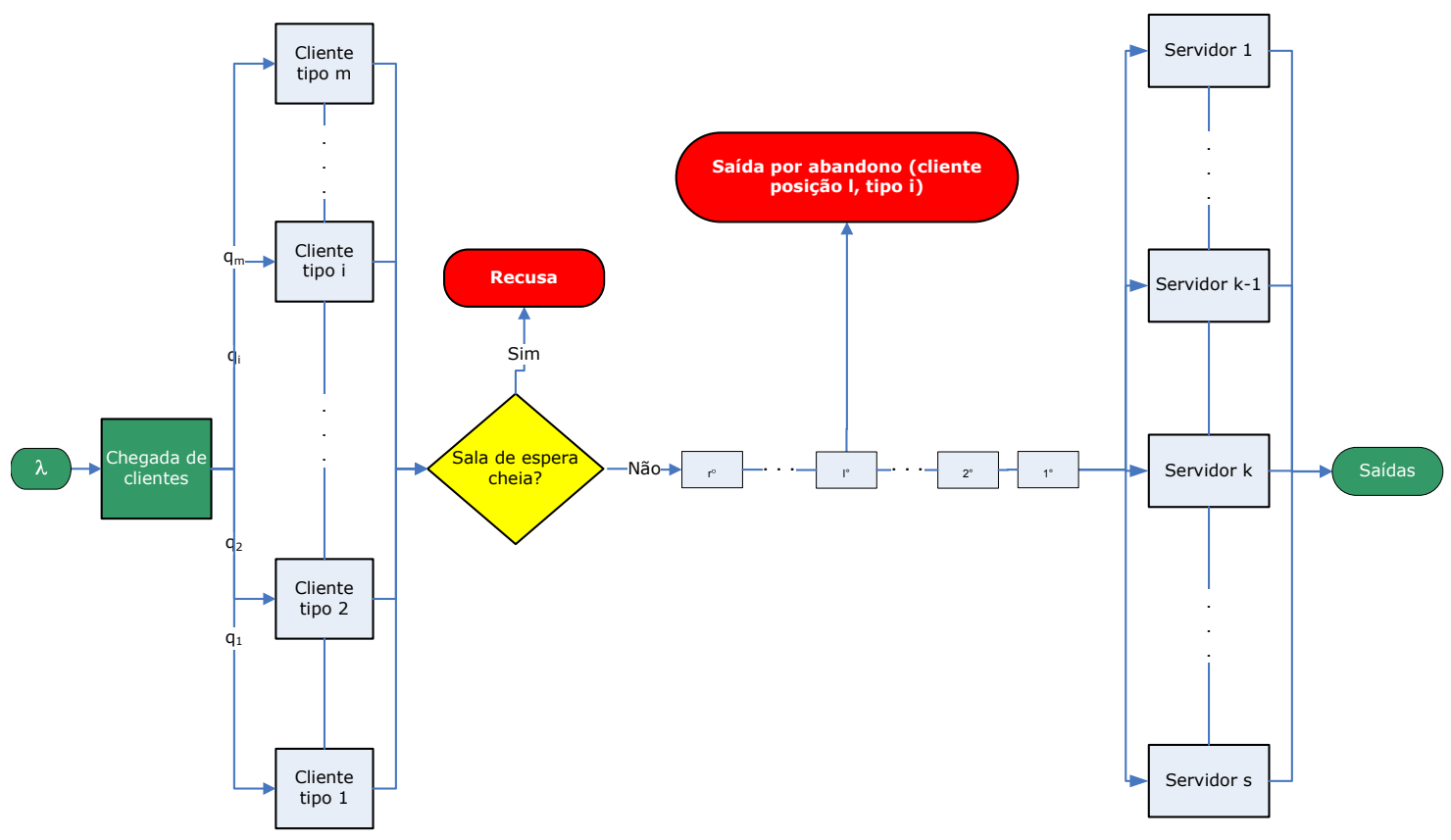

Figura 3.1: Sistema com $s$ servidores, sala de espera com $r$ clientes de $m$ tipos.

Seja $n_{i}$ o número de clientes tipo $i$ em serviço, com $i \in \mathcal{I}$.

Denotamos por $\widetilde{n}=\left(n_{1}, n_{2}, \ldots, n_{m}\right)$ o vetor com o número de clientes de cada tipo em serviço. Usaremos a notação $\tilde{n} 1$ para indicar a soma das coordenadas de $\widetilde{n}$. Isto é, $\widetilde{n} 1=n_{1}+\ldots+n_{m} \leqslant s$. O vetor $(0,0, \ldots, 0)$ será denotado por $\widetilde{0}$.

Vamos descrever o modelo $M / M^{i} / s / r+M^{i}(n)$ pelo conjunto finito de estados:

$$
\Omega=\left\{(\widetilde{n} ; \widetilde{e}) ; \quad \widetilde{n} 1 \leqslant s, i \in \mathcal{I}, n_{i} \geqslant 0 ; l=1,2, \ldots, r, e_{l} \in \mathcal{I} \cup\{0\}\right\}
$$

Para cada $t$ fixado seja $N(t)$ o estado desse sistema no instante $t$. Como chegadas, atendimentos e abandonos são Exponenciais, o processo estocástico $\mathcal{N}=\{N(t) ; t \geq 0\}$ é um processo Markoviano com espaços de estados enumerável $\Omega$.

Desta forma, $N \in \Omega$ é o estado do sistema em regime estacionário (omitimos o $t$ ) e um estado genérico pode ser representado por:

$$
N=(\widetilde{n} ; \widetilde{e})=\left(\left(n_{1}, n_{2}, \ldots, n_{m}\right) ;\left(e_{1}, e_{2}, \ldots, e_{r}\right)\right)
$$


Note inicialmente que chegadas ocorrem se pelo menos um $e_{l}$ é zero $(\operatorname{com} l=1,2, \ldots, r)$, ou seja, pelo menos a posição $r$ da fila não possui cliente. Caso contrário, a próxima chegada é recusada.

Para definir o operador e a taxa de transição para chegadas, assumimos que o estado $N$ é tal que há espaço na fila para a chegada. Ao ocorrer a chegada de um cliente tipo $i$, o sistema sai do estado $N$ e passa para o estado $T_{i}^{a}(N)$, que possui um cliente do tipo $i$ a mais no sistema. A taxa de transição de $N$ para $T_{i}^{a}(N)$ é dada por $\xi\left(N, T_{i}^{a}(N)\right)=\lambda q_{i}$. Podemos separar o estado $T_{i}^{a}(N)$ em dois casos, observando que a taxa de transição é a mesma para os dois casos.

Caso $N$ tenha espaço livre no servidor $(\tilde{n} 1<s)$, então o estado $T_{i}^{a}(N)$ possui $n_{i}+1$ clientes do tipo $i$ sendo atendidos. Caso $N$ tenha o servidor completo $(\widetilde{n} 1=s)$, então o estado $T_{i}^{a}(N)$ possui $s$ clientes sendo servidos e $l+1$ clientes aguardando na fila sendo $e_{l+1}=i$ para algum $l$, $l=1,2, \ldots, r-1$.

Note que ocorrendo um fim de serviço no sistema, os clientes que aguardam na fila são reposicionados. Por exemplo o cliente na posição 1 (que é o próximo a receber serviço) entra em serviço e os clientes que estão nas posições de 2 a $r$, passam uma posição para a frente. Ver Figura 3.2.

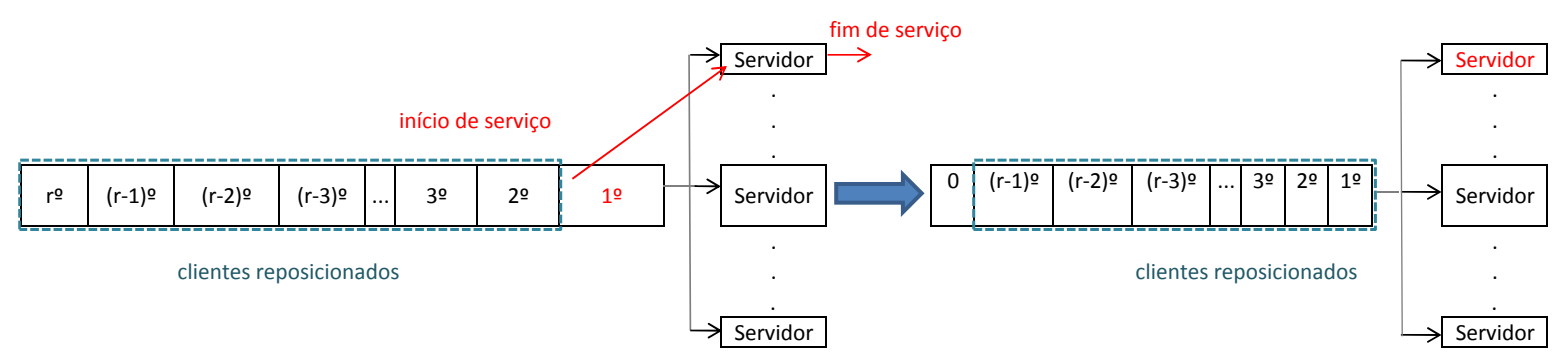

Figura 3.2: Reposicionamento de clientes após fim de serviço.

Na situação de ocorrência de um fim de serviço é necessário que tenham clientes sendo atendidos no sistema isto é $\widetilde{n} 1 \geqslant 1$.

Seja $1 \leqslant \widetilde{n} 1 \leqslant s, e_{l} \in \mathcal{I} \cup\{0\}, \operatorname{com} l=1,2, \ldots, r$ e considere que ocorre o fim de serviço de um cliente tipo $i$. Então o sistema sai do estado $N$ e passa ao estado $T_{i}^{b}(N)$ que possui um cliente a menos no sistema. A taxa de transição é dada por $\xi\left(N, T_{i}^{b}(N)\right)=n_{i} \mu_{i}$. Podemos separar o estado $T_{i}^{b}(N)$ em três casos, observando que todos os casos possuem a mesma taxa de transição. 
Caso $N$ não tenha fila $(\widetilde{e}=\widetilde{0})$, então o estado $T_{i}^{b}(N)$ possui $n_{i}-1$ clientes do tipo $i$ sendo servidos. Caso $N$ tenha fila $(\widetilde{e} \neq \widetilde{0})$ e o próximo cliente a ser servido é do mesmo tipo do cliente que finalizou serviço $\left(e_{1}=i\right)$, então o estado $T_{i}^{b}(N)$ vai continuar com $n_{i}$ clientes do tipo $i$ sendo servidos, porém com $l-1$ clientes aguardando na fila. Caso $N$ tenha fila $(\widetilde{e} \neq \widetilde{0})$ e o próximo cliente a ser servido é de tipo diferente do cliente que finalizou serviço $\left(e_{1} \neq i\right)$, então o estado $T_{i}^{b}(N)$ possui $n_{i}-1$ clientes do tipo $i$ e um cliente a mais do tipo $e_{1}$ sendo servido, ou seja, $\left(n_{1}, \ldots, n_{i}-1, \ldots, n_{e_{1}}+1, \ldots, n_{m}\right)$, e $l-1$ clientes aguardando na fila.

Da mesma forma que descrevemos o reposicionamento de clientes após um fim de serviço, o mesmo também ocorre após o abandono de um cliente. Assim, se um cliente está na posição $j+1$, após o abandono do j-ésimo cliente da fila, ele ocupará a posição $j$ da fila $(\operatorname{com} j=2, \ldots, r-1)$. Observando que se $j=1$, então o reposicionamento ocorrerá para as posições de 2 até $r$; e se $j=r$, não ocorrerá reposicionamento, apenas a última posição da fila ficará desocupada. O reposicionamento ocorre para clientes que estão atrás do cliente que abandonou. Ver Figura 3.3.

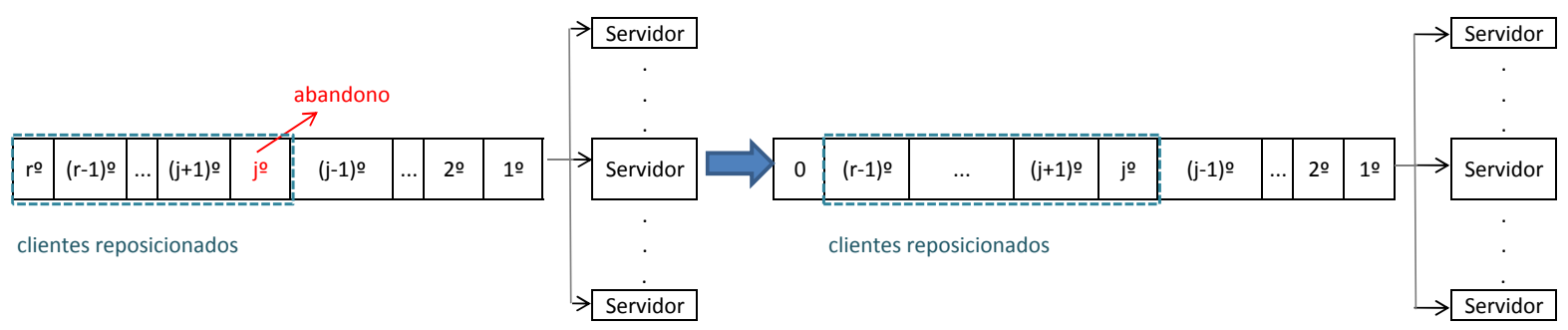

Figura 3.3: Reposicionamento de clientes após abandono.

Agora definiremos o operador e a taxa de transição para abandonos. Uma condição necessária neste caso é que exista fila, ou seja, o servidor está completo $(\widetilde{n} 1=s)$ e pelo menos $e_{1} \neq 0$. Se o cliente tipo $i$ na posição $j$ da fila abandona, então o sistema sai do estado $N$, e passa para o estado $T_{i, j}^{c}(N)$ que possui $l-1$ clientes aguardando na fila. A taxa de transição é definida por $\xi\left(N, T_{i, j}^{c}(N)\right)=\alpha_{i, j}$, em que $e_{j}=i$.

Seja $\widetilde{n}=\left(n_{1}, n_{2}, \ldots, n_{i}, \ldots, n_{m}\right)$ e $e_{l} \in \mathcal{I} \cup\{0\}$, com $1 \leqslant j \leqslant l \leqslant r$. Definimos a seguinte notação:

$\widetilde{n}-1_{i}=\left(n_{1}, n_{2}, \ldots, n_{i}-1, \ldots, n_{m}\right) ;$ 
$\widetilde{n}+1_{i}=\left(n_{1}, n_{2}, \ldots, n_{i}+1, \ldots, n_{m}\right)$;

$\widetilde{e}-1_{i, l}=$ configuração de fila de $\widetilde{e}$ sem o cliente da posição $l$, que era do tipo $i$;

$\widetilde{e}+1_{i, l+1}=$ a fila $\widetilde{e}$ adicionada de um cliente tipo $i$, na posição $l+1$. Observando que neste caso, $l$ pode ser no máximo igual a $r-1$, pois é necessário que seja possível a chegada de um cliente, $\mathrm{I}_{\left\{n_{i}>0\right\}}= \begin{cases}0, & \text { se } n_{i} \leqslant 0 \\ 1, & \text { se } n_{i}>0 .\end{cases}$

Seja $\pi$ a probabilidade estacionária do modelo $M / M^{i} / s / r+M^{i}(n)$. Vamos definir as equações de balanço para esse modelo igualando os fluxos de probabilidade de saída e entrada em cada de estado.

- Caso 1: Seja

$$
N \in \mathcal{M}_{1}=\{(\widetilde{n} ; \widetilde{e}) \in \Omega: 0 \leqslant \widetilde{n} 1<s, \widetilde{e}=\widetilde{0}\}
$$

Para $\forall i, n_{i}>0$ definimos

$$
A_{i} \in \mathcal{A}_{i}=\left\{\left(\widetilde{n}-1_{i} ; \widetilde{0}\right):(\widetilde{n} ; \widetilde{0}) \in \mathcal{M}_{1}\right\}
$$

e

$$
B_{i} \in \mathcal{B}_{i}=\left\{\left(\widetilde{n}+1_{i} ; \widetilde{0}\right):(\widetilde{n} ; \widetilde{0}) \in \mathcal{M}_{1}\right\}
$$

O conjunto de estados $\mathcal{M}_{1}$ descrito acima é formado pelos estados $N$ que satisfazem as condições de não possuir fila e conter no máximo $s-1$ clientes em serviço.

O conjunto de estados $\mathcal{A}_{i}$ é formado pelos estados $A_{i}$ que possuem a configuração do conjunto de estados $\mathcal{M}_{1}$, com um cliente do tipo $i$ a menos. O conjunto de estados $\mathcal{B}_{i}$ é formado pelos estados $B_{i}$ que possuem a configuração do conjunto de estados $\mathcal{M}_{1}$, com um cliente do tipo $i$ a mais.

As transicões de entrada e saída para um estado $N \in \mathcal{M}_{1}$ são apresentados na Figura 3.4 e a equação de balanço correspondente é definida pela Equação 3.7 .

$$
\pi_{N} \sum_{i=1}^{m}\left(n_{i} \mu_{i} \mathrm{I}_{\left\{n_{i}>0\right\}}+\lambda q_{i}\right)=\sum_{i=1}^{m} \pi_{A_{i}} \lambda q_{i}+\sum_{i=1}^{m} \pi_{B_{i}} n_{i} \mu_{i} \mathrm{I}_{\left\{n_{i}>0\right\}} .
$$

- Caso 2: Seja

$$
N \in \mathcal{M}_{2}=\{(\widetilde{n} ; \widetilde{0}) \in \Omega: \widetilde{n} 1=s\}
$$




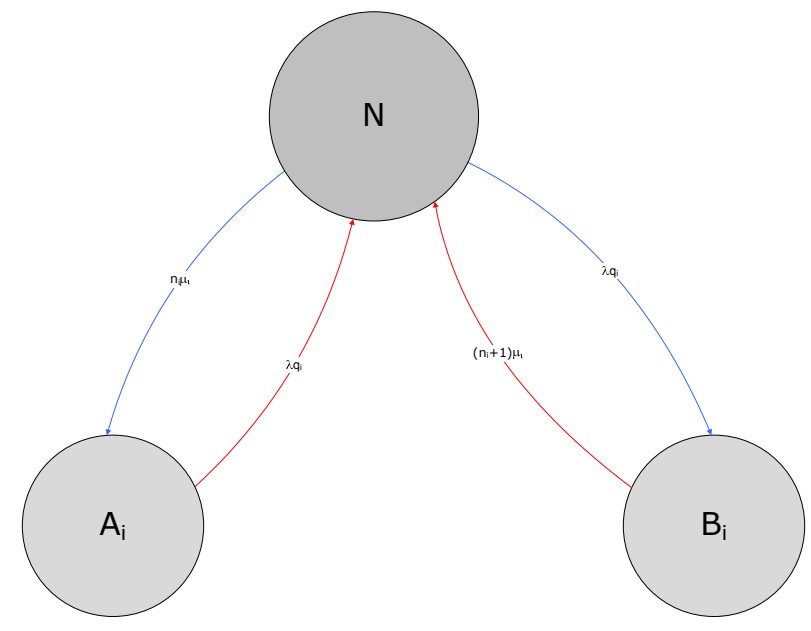

Figura 3.4: Fluxo caso 1.

Para $\forall i, n_{i}>0$ definimos

$$
\begin{gathered}
C_{i} \in \mathcal{C}_{i}=\left\{\left(\widetilde{n}-1_{i} ; \widetilde{0}\right):(\widetilde{n} ; \widetilde{0}) \in \mathcal{M}_{2}\right\}, \\
D_{i} \in \mathcal{D}_{i}=\left\{\left(\widetilde{n} ; \widetilde{0}+1_{i, 1}\right):(\widetilde{n} ; \widetilde{0}) \in \mathcal{M}_{2}\right\}, \\
U_{i} \in \mathcal{U}_{i}=\left\{\left(\widetilde{n}-1_{i}+1_{u} ; \widetilde{0}+1_{i, 1}\right):(\widetilde{n} ; \widetilde{0}) \in \mathcal{M}_{2}\right\}, \quad u \neq i .
\end{gathered}
$$

$\mathrm{O}$ conjunto de estados $\mathcal{M}_{2}$ é formado pelos estados $N$ que satisfazem as condições de não possuir fila e conter exatamente $s$ clientes em serviço.

Os conjuntos de estados $\mathcal{C}_{i}, \mathcal{D}_{i}$ e $\mathcal{U}_{i}$ são definidos em decorrência do conjunto de estados $\mathcal{M}_{2}$. Assim, o conjunto de estados $\mathcal{C}_{i}$ é formado pelos estados $C_{i}$ que possuem a configuração do conjunto de estados $\mathcal{M}_{2}$, com um cliente do tipo $i$ a menos.

O conjunto de estados $\mathcal{D}_{i}$ é formado pelos estados $D_{i}$ que possuem a configuração do conjunto de estados $\mathcal{M}_{2}$, com um cliente do tipo $i$ a mais na posição 1 da fila. O conjunto de estados $\mathcal{U}_{i}$ é formado pelos estados $U_{i}$ que possuem a configuração do conjunto de estados $\mathcal{M}_{2}$, com um cliente do tipo $i$ a menos, um cliente tipo $u$ a mais no servidor e mais um cliente tipo $i$ na posição 1 da fila.

O fluxo de probabilidade de saída e entrada do estado $N \in \mathcal{M}_{2}$ é dado pela Figura 3.5 e a 
equação de balanço é definida pela Equação 3.12.

$$
\begin{array}{r}
\pi_{N} \sum_{i=1}^{m}\left(n_{i} \mu_{i} \mathrm{I}_{\left\{n_{i}>0\right\}}+\lambda q_{i}\right)= \\
\sum_{i=1}^{m} \pi_{C_{i}} \lambda q_{i}+\sum_{i=1}^{m} \pi_{D_{i}}\left(n_{i} \mu_{i} \mathrm{I}_{\left\{n_{i}>0\right\}}+\alpha_{i, 1}\right)+\sum_{u=1}^{m} \pi_{U_{i}} n_{u} \mu_{u} \mathrm{I}_{\left\{n_{u}>0, u \neq i\right\}} .
\end{array}
$$

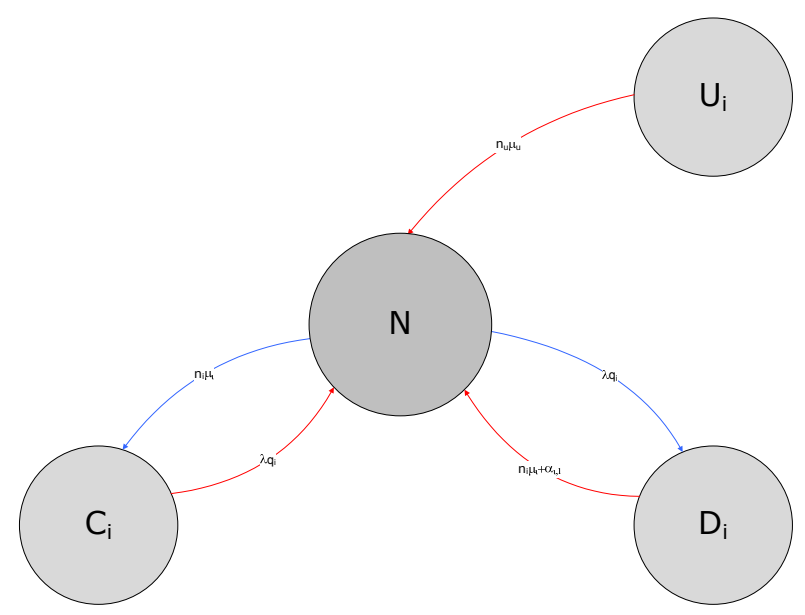

Figura 3.5: Fluxo caso 2.

- Caso 3: Seja

$$
N \in \mathcal{M}_{3}=\left\{(\widetilde{n} ; \widetilde{e}) \in \Omega: \widetilde{n} 1=s ; e_{l+1}=0\right\}
$$

$\operatorname{com} l=1, \ldots, r-1$.

Para $\forall i, n_{i}>0, e_{l} \in \mathcal{I}, l=1, \ldots, r-1, j=1, \ldots, l, j^{*}=1, \ldots, r$ definimos

$$
\begin{gathered}
F_{e_{l}} \in \mathcal{F}_{e_{l}}=\left\{\left(\widetilde{n} ; \widetilde{e}-1_{e_{l}, l}\right):(\widetilde{n} ; \widetilde{e}) \in \mathcal{M}_{3}\right\} \\
G_{e_{j^{*}}, j^{*}} \in \mathcal{G}_{e_{j^{*}, j^{*}}}=\left\{\left(\widetilde{n} ;\left(e_{1}, \ldots, e_{j}, e_{j^{*}}, e_{j+1}, 0, \ldots, 0\right)\right):(\widetilde{n} ; \widetilde{e}) \in \mathcal{M}_{3}\right\}, \\
L_{e_{l+1}} \in \mathcal{L}_{e_{l+1}}=\left\{\left(\widetilde{n} ; \widetilde{e}+1_{e_{l+1}, l+1}\right):(\widetilde{n} ; \widetilde{e}) \in \mathcal{M}_{3}\right\},
\end{gathered}
$$




$$
P_{e_{j}} \in \mathcal{P}_{e_{j}}=\left\{\left(\widetilde{n} ; \widetilde{e}-1_{e_{j}, j}\right):(\widetilde{n} ; \widetilde{e}) \in \mathcal{M}_{3}\right\}
$$

O conjunto de estados $\mathcal{M}_{3}$ é formado pelos estados $N$ que satisfazem as seguintes condições: contém exatamente $s$ clientes em serviço e possui fila, onde o número de clientes na fila é $l$ $(1 \leqslant l \leqslant r-1)$.

Os conjuntos de estados $\mathcal{F}_{e_{l}}, \mathcal{G}_{e_{j^{*}}, j^{*}}, \mathcal{L}_{e_{l+1}}$ e $\mathcal{P}_{i}$ são definidos em decorrência do conjunto de estados $\mathcal{M}_{3}$. Assim, o conjunto de estados $\mathcal{F}_{e_{l}}$ é formado pelos estados $F_{i}$ que possuem a configuração do conjunto de estados $\mathcal{M}_{3}$, com o cliente da posição $l$ e do tipo $e_{l}$ a menos (deve ser observado que no conjunto de estados $\mathcal{M}_{3}, l$ é a última posição da fila que possui cliente).

O conjunto de estados $\mathcal{G}_{e_{j^{*}}}$ é formado pelos estados $G_{e_{j^{*}}}$ que possuem a configuração do conjunto de estados $\mathcal{M}_{3}$, com um cliente a mais na fila, na posição $j^{*}$ do tipo $e_{j^{*}}$. O conjunto de estados $\mathcal{L}_{e_{l+1}}$ é formado pelos estados $L_{e_{l+1}}$ que possuem a configuração do conjunto de estados $\mathcal{M}_{3}$, mais um cliente tipo $e_{l+1}$ na posição $l+1$ da fila. $\mathrm{O}$ conjunto de estados $\mathcal{P}_{e_{j}}$ é formado pelos estados $P_{e_{j}}$ que possuem a configuração do conjunto de estados $\mathcal{M}_{3}$, com um cliente na posição $j$ do tipo $e_{j}$ a menos na fila.

O fluxo de probabilidade de saída e entrada do estado $N \in \mathcal{M}_{3}$ é dado pela Figura 3.6 e a equação de balanço é definida pela Equação 3.18.

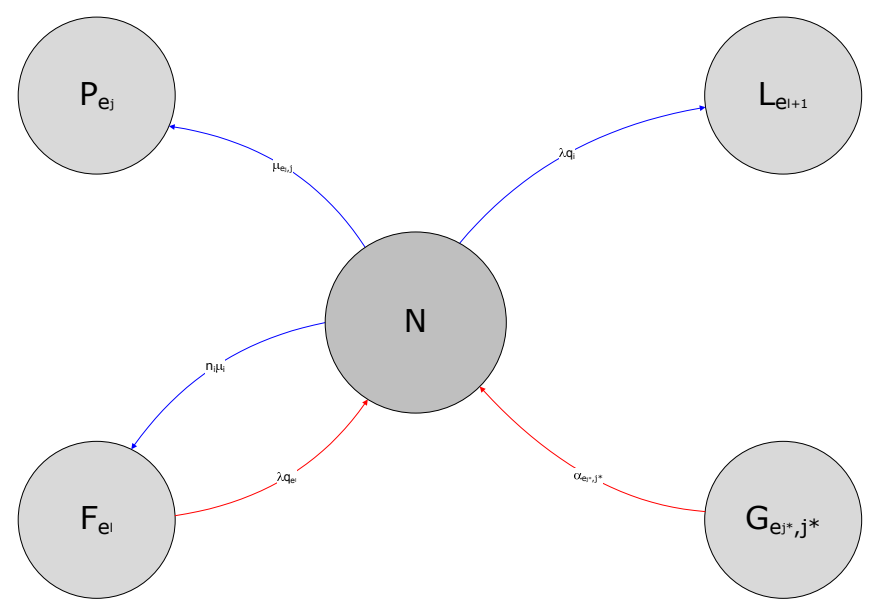

Figura 3.6: Fluxo caso 3. 


$$
\pi_{N}\left[\sum_{i=1}^{m}\left(n_{i} \mu_{i} \mathrm{I}_{\left\{n_{i}>0\right\}}+\lambda q_{i}\right)+\sum_{j=1}^{l} \alpha_{e_{j}, j}\right]=\pi_{F_{e_{l}}} \lambda q_{e_{l}}+\sum_{G_{j^{*}=1}}^{m} \sum_{j^{*}=1}^{l} \pi_{G_{e_{j^{*}, j^{*}}}} \alpha_{e_{j^{*}, j^{*}}}
$$

$\operatorname{com} l=1, \ldots, r, j=1, \ldots, l$ e $j^{*}=1, \ldots, l$.

- Caso 4: Seja

$$
N \in \mathcal{M}_{4}=\left\{(\widetilde{n} ; \widetilde{e}) \in \Omega: \widetilde{n} 1=s ; e_{r} \neq 0\right\}
$$

Para $\forall e_{l}$ definimos

$$
\begin{aligned}
& H_{e_{r}} \in \mathcal{H}_{e_{r}}=\left\{\left(\widetilde{n} ; \widetilde{e}-1_{e_{r}, r}\right):(\widetilde{n} ; \widetilde{e}) \in \mathcal{M}_{4}\right\}, \\
& T_{e_{j^{*}, j^{*}}} \in \mathcal{T}_{e_{j^{*}, j^{*}}}=\left\{\left(\widetilde{n} ; \widetilde{e}-1_{\left.e_{j^{*}, j^{*}}\right)}:(\widetilde{n} ; \widetilde{e}) \in \mathcal{M}_{4}\right\}\right.
\end{aligned}
$$

$\operatorname{com} j^{*}=1, \ldots, r$

$$
Q_{e_{1}} \in \mathcal{Q}_{e_{1}}=\left\{\left(\widetilde{n}+1_{e_{1}} ; \widetilde{e}-1_{e_{1}, 1}\right):(\widetilde{n} ; \widetilde{e}) \in \mathcal{M}_{4}\right\}
$$

O conjunto de estados $\mathcal{M}_{4}$ é formado pelos estados $N$ que satisfazem as seguintes condições: contém exatamente $s$ clientes em serviço e $r$ clientes na fila.

Os conjuntos de estados $\mathcal{H}_{e_{r}}, \mathcal{T}_{e_{j^{*}} j^{*}}$ e $\mathcal{Q}_{e_{1}}$ são definidos em decorrência do conjunto de estados $\mathcal{M}_{4}$. Assim, o conjunto de estados $\mathcal{H}_{e_{r}}$ é formado pelos estados $H_{e_{r}}$ que possuem a configuração do conjunto de estados $\mathcal{M}_{4}$, com o cliente da posição $r$ e do tipo $e_{r}$ a menos.

O conjunto de estados $\mathcal{T}_{e^{*}, j^{*}}$ é formado pelos estados $T_{e_{j^{*}}, j^{*}}$ que possuem a configuração do conjunto de estados $\mathcal{M}_{4}$, com um cliente a menos na fila, na posição $j^{*}$ do tipo $e_{j^{*}}$. O conjunto de estados $\mathcal{Q}_{e_{1}}$ é formado pelos estados $Q_{e_{1}}$ que possuem a configuração do conjunto de estados $\mathcal{M}_{4}$, menos um cliente tipo $e_{1}$ na posição 1 da fila.

O fluxo de entradas e saídas do estado $\mathcal{M}_{4}$ é dado pela Figura 3.7 e a equação de balanço é definida pela Equação 3.23. 


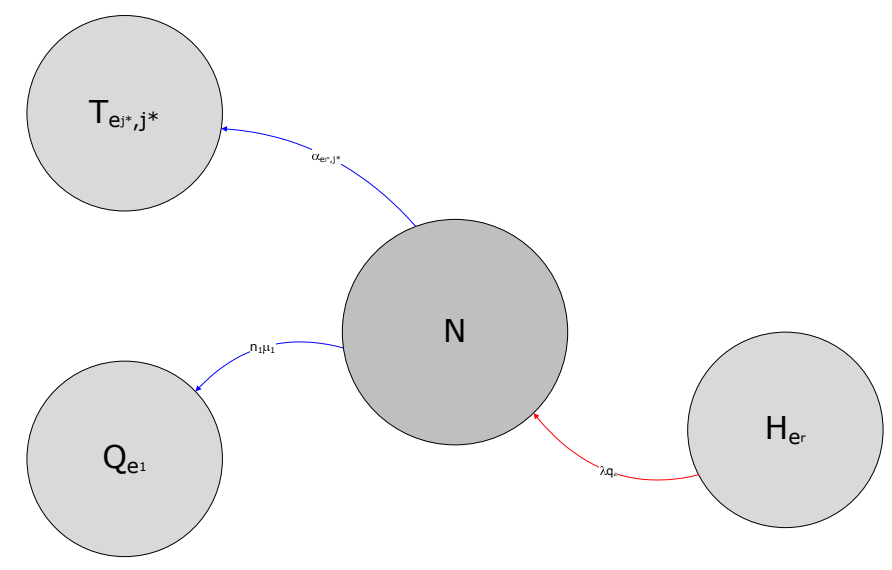

Figura 3.7: Fluxo caso 4.

$$
\pi_{N}\left[\sum_{i=1}^{m} n_{i} \mu_{i} \mathrm{I}_{\left\{n_{i}>0\right\}}+\sum_{j=1}^{r} \alpha_{e_{j}, j}\right]=\pi_{H_{e_{r}}} \lambda q_{e_{r}}
$$

Utilizando as equações de balanço e a restrição de normalização $\left(\sum_{N \in \Omega} \pi_{N}=1\right)$, obtemos um sistema que apresenta solução única. Assumindo conhecidos $\pi_{N}, N \in \Omega$, desenvolvemos na próxima seção as medidas de desempenho do modelo $M / M^{i} / s / r+M^{i}(n)$.

\subsection{Medidas de Desempenho $-M / M^{i} / s / r+M^{i}(n)$}

As medidas de desempenho obtidas por Whitt em [15] e descritas na Seção 2.2, são agora estendidas para o caso de diferentes tipos de clientes.

Assumindo que nosso sistema possui $m$ tipos de clientes ele terá $m$ diferentes distribuições para as taxas de abandono e de serviço. Como a função taxa de falha é definida pela paciência de cada cliente, então cada tipo de cliente terá uma função taxa de falha diferente, definida por

$$
h_{i}(t)=\frac{f_{i}(t)}{F_{i}^{c}(t)}, \quad t \geqslant 0,
$$

em que $f_{i}(t)$ e $F_{i}^{c}(t)$ são respectivamente, a função densidade de probabilidade e a função distribuição de sobrevivência da paciência do cliente tipo $i$, com $i \in \mathcal{I}$.

De forma análoga à Seção 2.4 os clientes são contados a partir do fim da fila, de maneira que o último cliente a ser atendido recebe o número um, o penúltimo cliente o número dois, e assim por diante, até o primeiro cliente a ser atendido receber o número $l(1 \leqslant l \leqslant r)$. O sistema é observado 
considerando um total de $k$ clientes, sendo que $s$ clientes sendo servidos e $l$ clientes aguardando na fila, onde novas chegadas são ignoradas e a média de chegadas é $1 / \lambda$, independente do tipo de cliente.

Assim, a taxa de abandono é aproximada por

$$
\alpha_{i, j} \equiv h_{i}(j / \lambda), \quad i \in \mathcal{I},
$$

onde $j$ é a posição do cliente, contado a partir do fim da fila $(1 \leqslant j \leqslant l), \lambda$ é a taxa de chegada, $i$ o tipo do cliente e $h_{i}$ dada por (3.24).

Vamos definir o conjunto dos estados de $N_{i, l} \in \Omega$ que contém $s$ clientes em serviço e $l$ clientes na fila, sendo que o l-ésimo cliente que chegou na fila é do tipo $i$, como

$$
\mathcal{N}_{i, l}=\left\{N_{i, l} \in \Omega: e_{l} \neq 0, \widetilde{n} 1=s ; 1 \leqslant l \leqslant r\right\}
$$

ou seja, o estado $N_{i, l}$ tem $l-1$ clientes na fila acrescido de um cliente tipo $i$ na posição $l$ da fila.

Então para $N \in \mathcal{N}_{i, l}$

$$
N_{i, l}=\left(\left(n_{1}, n_{2}, \ldots, n_{m}\right) ;\left(e_{1}, e_{2}, \ldots, e_{l-1}, i, 0, \ldots, 0\right)\right)
$$

Como já foi mencionado anteriormente, neste capítulo além de considerar a posição do cliente na fila, consideramos o tipo do cliente. A posição do cliente na fila é contada a partir do fim da fila, enquanto o tipo do cliente é observado a partir do início da fila. Por este motivo, vamos descrever a relação entre a posição do cliente na fila e seu tipo.

Vamos considerar o estado $N \in \mathcal{N}_{i, l}$, agora analisando apenas a porção da fila. Observe na Figura 3.8 que o último (l-ésimo) cliente a chegar na fila, é do tipo $e_{l}$ e sua posição na fila é 1 . O cliente na posição $j$ da fila (contado a partir do fim) é do tipo $e_{l-j+1}$ e, ainda, o cliente na posição $l$ é do tipo $e_{1}$.

Definimos a taxa de abandono da fila para algum estado $N \in \mathcal{N}_{i, l}$, por:

$$
\delta_{N_{i, l}}=\sum_{f=1}^{l} \alpha_{e_{l-f+1}, f}
$$

em que $1 \leqslant f \leqslant l \leqslant r$

Agora analisamos a porção de clientes sendo servidos em um dos estados $N_{i, l} \in \mathcal{N}_{i, l}$. O vetor $\left(n_{1}, n_{2}, \ldots, n_{m}\right)$, que representa o número de clientes de cada tipo que estão sendo servidos, é conhecido . Então é possível definir a taxa de saída por fim de serviço como $\sum_{i=1}^{m} n_{i} \mu_{i}$. 


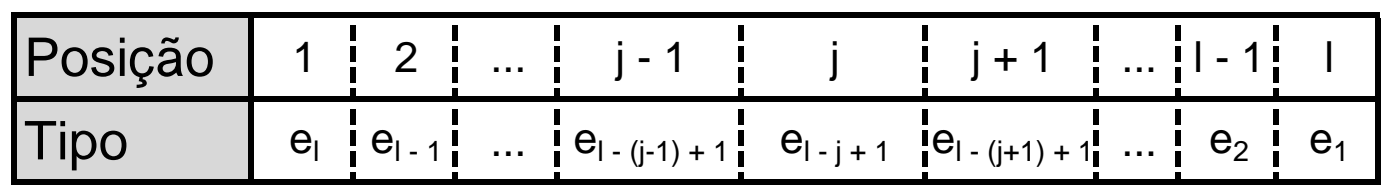

Figura 3.8: Tipo e posição de clientes na fila.

Assim definimos a taxa de saída do sistema (por abandono ou por fim de serviço) no estado $N \in \Omega$, que contém $k$ clientes, como

$$
\mu_{N}=\left\{\begin{array}{cc}
\sum_{i=1}^{m} n_{i} \mu_{i}, & \text { para } N \text { em que } 1 \leqslant k \leqslant s ; \\
\sum_{i=1}^{m} n_{i} \mu_{i}+\sum_{f=1}^{k} \alpha_{e_{l-f+1}, f}, & \text { para } N \text { em que } s<k \leqslant s+r ;
\end{array}\right.
$$

onde $k$ é o número de clientes no sistema, com $l$ clientes na fila $(1 \leqslant l \leqslant r), s$ clientes em serviço e $m$ são os possíveis tipos de clientes; $\mu_{i}$ a taxa de atendimento do cliente tipo $i$.

Assim, a probabilidade do sistema estar completo indicará a probabilidade de uma chegada ser recusada, isto é,

$$
P(\text { Recusa })=\sum_{N \in \mathcal{M}_{4}} \pi_{N}
$$

onde $\mathcal{M}_{4}$ é definido por 3.19 .

Seja $\Omega^{*}$ o conjunto de todos os estados de $\Omega$ que possuem espaço na fila, ou seja, $\Omega^{*}=\Omega-\mathcal{M}_{4}$.

Desta forma, definimos a probabilidade vista por um cliente que ao entrar encontra o sistema no estado $N$ por:

$$
\pi_{N}^{a}=\frac{\pi_{N}}{(1-P(\text { Recusa }))}, N \in \Omega^{*}
$$


Para $0 \leqslant k \leqslant s-1$, temos que a probabilidade do cliente entrar no sistema e ser atendido no mesmo instante é dada por

$$
P(\text { Atendimento Imediato })=P(W=0)=\sum_{N \in \mathcal{M}_{1}^{*}} \pi_{N}^{a},
$$

sendo $\mathcal{M}_{1}^{*}$ o conjunto de estados pertencentes ao conjunto de estados $\mathcal{M}_{1} \in \Omega^{*}$.

Vamos calcular agora a probabilidade do l-ésimo cliente que chegou na fila, abandoná-la após $j-1$ eventos de partidas.

Deve ser observado que após a chegada do l-ésimo cliente, assumimos que futuras chegadas são desconsideradas e as taxas de abandonos dos clientes na fila são conhecidas, ou seja, o l-ésimo cliente, que é tipo $i$, possui taxa de abandono $\alpha_{i, l} \equiv h_{i}(l / \lambda)$. Para estudar o estado após $j-1$ saídas, é necessário observar o sistema dinâmico no tempo. Assim, como estamos considerando o tempo, deve ser considerado que os clientes que já estão na fila, aumentam seus tempos de espera a cada instante. Então passa ser necessário que as taxas de paciência destes clientes sejam alteradas conforme o tempo que o cliente já esperou. Então uma aproximação adicional é feita, assumimos $1 / \lambda$ como o tempo entre cada intervalo de saída, desta forma, após $j-1$ partidas, se o $l$-ésimo cliente ainda estiver na fila, sua taxa de paciência passa a ser $\alpha_{i, l+(j-1)} \equiv h_{i}((l+(j-1)) / \lambda)$.

Com a aproximação acima resolvemos o problema da dependência da taxa de paciência pelo tempo e definimos a evolução da taxa de paciência. Contudo, ocorrendo $j-1$ saídas, a evolução do sistema depende se ocorreram saídas por fim de serviço ou por abandono. Desta forma, mais uma aproximação é feita e assumimos que todas as $j-1$ saídas foram por fim de serviço. Assim, os primeiros $j-1$ clientes aguardando na fila, inicialmente com taxas de abandono $\left(\alpha_{e_{l-(j-1)+1}, j-1}, \ldots, \alpha_{e_{1}, l}\right)$, entram em serviço. Então os demais $l-(j-1)$ clientes, com taxas de abandono iniciais $\left(\alpha_{e_{l}, 1}, \ldots, \alpha_{e_{j}, l-(j-1)}\right)$, têm suas posições acrescidas de $j-1$ e passam a ter taxas de paciência $\left(\alpha_{e_{l}, j}, \ldots, \alpha_{e_{j}, l}\right)$.

Uma observação bastante importante a ser feita é que a posição do cliente muda, mas seu tipo permanece aquele definido no momento de entrada no sistema.

Para se saber o estado do sistema após um certo número de saídas a partir do estado $N$, seria necessário acompanhar a evolução do número de atendimentos de cada tipo. Isto envolve os tipos dos clientes em fila e os eventuais abandonos ocorridos. Dessa forma, é bastante complicado estabelecer a configuração do número de clientes de cada tipo em serviço após várias saídas. Assim, duas possíveis alternativas para contornar esta dificulade seriam:

(1) Considerar que após as $j-1$ ocorrências de saídas temos o serviço com o mesmo vetor $\left(n_{1}, n_{2}, \ldots, n_{m}\right)$ igual, após as $j-1$ ocorrências de saídas, e a taxa total de fim de serviço ficaria 
$\sum_{i=1}^{m} n_{i} \mu_{i}$

(2) Assumir uma taxa média por servidor considerando os tipos, ou seja, $s \sum_{i=1}^{m} \mu_{i} q_{i}$ seria a taxa total de fim de serviço.

Vamos considerar nos cálculos abaixo a alternativa (2), pois nos pareceu menos restritiva que a altenativa (1).

Também foi observada a dificuldade em estabelecer a configuração dos clientes que aguardam na fila, após várias saídas de clientes do sistema. Assim uma segunda aproximação é proposta:

Assumimos uma taxa média por abandono, ou seja, $\sum_{i=1}^{m} \sum_{f=j+1}^{l} q_{i} \alpha_{i, f}+\alpha_{i, j}$ seria a taxa total de abandono.

Seja $N_{i, l}$ o estado do sistema que tem $l$ clientes em fila, sendo que $e_{l}=i$. Definimos $\gamma_{N_{i, l}, j}$ como a probabilidade de um cliente tipo $i$ que entrou na fila, fazendo o sistema ficar com $k$ clientes e abandonar quando estiver na j-ésima posição da fila (contada a partir do fim). A partir dos comentarios acima temos:

$$
\gamma_{N_{i, l}, j} \approx \frac{\alpha_{i, j}}{s \sum_{i=1}^{m} \mu_{i} q_{i}+\sum_{i=1}^{m} \sum_{f=j+1}^{l} q_{i} \alpha_{i, f}+\alpha_{i, j}}
$$

Logo a probabilidade do cliente no estado $N_{i, l}$ não abandonar o sistema até ser servido é:

$$
\Gamma_{N_{i, l}}=\prod_{j=1}^{l}\left(1-\gamma_{N_{i, l}, j}\right)=\left(1-\gamma_{N_{i, l}, 1}\right)\left(1-\gamma_{N_{i, l}, 2}\right) \ldots\left(1-\gamma_{N_{i, l}, i, l}\right)
$$

para $\gamma_{N_{i, l}, j}$ em (3.31).

Assim a probabilidade do cliente que chegou na fila na posição $l$ não abandonar até ser servido (independente de seu tipo) é dada por:

$$
\Gamma_{N_{., l}}=\sum_{i=1}^{m} q_{i} \Gamma_{N_{i, l}}
$$

Seja $\mathcal{M}^{*}=\mathcal{M}_{2} \cup \mathcal{M}_{3}$, ou seja, todos os estados que possuem pelo menos $s$ clientes sendo servidos, podendo existir fila ou não. Desta forma calculamos aproximadamente, a probabilidade 
de um cliente que entrou no sistema ser atendido dada por

$$
P(S)=\left(\sum_{N \in \mathcal{M}_{1}^{*}} \pi_{N}^{a}\right)+\sum_{l=0}^{r-1} \sum_{N \in \mathcal{M}^{*}} \pi_{N}^{a} \Gamma_{N_{., l+1}}
$$

para $\Gamma_{N_{., l+1}}$ definido em (3.33).

Novamente, como no Capítulo 2, podemos expressar a probabilidade de um cliente que entra no sistema e o abandona como

$$
P(A)=1-P(S)
$$

Para um estado $N_{i, l}$, vamos definir $m_{N_{i, l}, j}$ como sendo o tempo médio entre o $(j-1)$-ésimo e j-ésimo eventos de partida. Temos:

$$
m_{N_{i, l}, j} \approx \frac{1}{s \sum_{i=1}^{m} \mu_{i} q_{i}+\sum_{i=1}^{m} \sum_{f=j+1}^{l} q_{i} \alpha_{i, f}+\alpha_{i, j}}
$$

$\mathrm{e}$

$$
m_{N_{, l}, j}=\sum_{i=1}^{m} q_{i} m_{N_{i, l}, j}
$$

Os dois primeiros momentos do tempo médio do cliente na fila que é atendido são dados por

$$
E[W ; S]=\sum_{l=0}^{r-1} \sum_{N \in \mathcal{M}^{*}} \pi_{N}^{a} \Gamma_{N_{., l+1}} \sum_{j=1}^{l+1} m_{N_{., l+1}, j}
$$

e

$$
E\left[W^{2} ; S\right]=\sum_{l=0}^{r-1} \sum_{N \in \mathcal{M}^{*}} \pi_{N}^{a} \Gamma_{N_{., l+1}}\left(V_{N_{., l+1}}+M_{N_{., l+1}}^{2}\right)
$$

em que

$$
V_{N_{., l+1}} \equiv \sum_{j=1}^{l+1} m_{N_{., l}, j}^{2} \quad e \quad M_{N_{., l+1}} \equiv \sum_{j=1}^{l+1} m_{N_{., l}, j} .
$$

Então,

$$
E(W \mid S)=\frac{E[W ; S]}{P(S)}
$$


e

$$
E\left(W^{2} \mid S\right)=\frac{E\left[W^{2} ; S\right]}{P(S)}
$$

Assim, a variância condicional é

$$
\operatorname{Var}(W \mid S) \equiv E\left(W^{2} \mid S\right)-(E(W \mid S))^{2}
$$

Como indicado no Capítulo 2, usaremos aqui também as transformadas de Laplace para obter as soluções numéricas de $P(W \leqslant t \mid S)$.

Uma outra forma de cálculo para a probabilidade de abandonar antes do início do serviço, para um cliente que chega no sistema e ocupa a posição $l+1$ da fila $(0 \leqslant l \leqslant r-1)$, é dada por:

$$
P(A)=\sum_{l=0}^{r-1} \sum_{N \in \mathcal{M}_{*}} \pi_{N}^{a}\left(1-\Gamma_{N_{., l+1}}\right) .
$$

Seja $W_{N_{i, l}}$ o tempo de espera de um cliente (do tipo $i$ ) que ao entrar no sistema encontra o estado $N$, com $l-1$ clintes na fila, e ocupa a posição $l$ na fila. Temos

O tempo médio no sistema com $k=s+l$ clientes, do l-ésimo abandonar

$$
\begin{array}{r}
E\left[W_{N_{i, l}} 1_{A}\right]=\gamma_{N_{i, l}, 1} m_{N_{i, l}, 1}+\left(1-\gamma_{N_{i, l}, 1}\right) \gamma_{N_{i, l}, 2}\left(m_{N_{i, l}, 1}+m_{N_{i, l}, 2}\right)+ \\
+\left(1-\gamma_{N_{i, l}, 1}\right)\left(1-\gamma_{N_{i, l}, 2}\right) \gamma_{N_{i, l}, 3}\left(m_{N_{i, l}, 1}+m_{N_{i, l}, 2}+m_{N_{i, l}, 3}\right)+\ldots+ \\
+\left(1-\gamma_{N_{i, l}, 1}\right) \ldots\left(1-\gamma_{N_{i, l}, l-1}\right) \gamma_{N_{i, l}, l}\left(m_{N_{i, l}, 1}+\ldots+m_{N_{i, l}, l}\right)
\end{array}
$$

em que $1 \leqslant l \leqslant r$ e $1 \leqslant i \leqslant m$.

O tempo médio de abandono $\left(E\left[W_{N_{i, l}} 1_{A}\right]\right)$ pode ser entendido como: o l-ésimo cliente a chegar na fila abandona na $1^{\underline{a}}$ saída, ou na $2^{\underline{a}}$ saída, ou na l-ésima saída. Para a $1^{\underline{a}}$ saída temos a probabilidade dada por $\gamma_{N_{i, l}, 1}$ com tempo $m_{N_{i, l}, 1}$. Para as outras saídas o argumento é similar, lembrando que é preciso considerar a probabilidade de não ter abandonado na etapas anteriores e incluir os tempos de espera entre as sucessivas saídas.

Então

$$
E\left[W_{N_{., l}} 1_{A}\right]=\sum_{i=1}^{m} q_{i} E\left[W_{N_{i, l}} 1_{A}\right]
$$


$\mathrm{e}$

$$
E\left[W 1_{A}\right]=\sum_{l=1}^{r} \sum_{N \in \mathcal{M}^{*}} \pi_{N}^{a} E\left[W_{N_{., l}} 1_{A}\right] .
$$

De modo análogo temos:

$$
\begin{array}{r}
E\left[W_{N_{i, l}}^{2} 1_{A}\right]=\gamma_{N_{i, l}, 1} 2 m_{N_{i, l}, 1}^{2}+\left(1-\gamma_{N_{i, l}, 1}\right) \gamma_{N_{i, l}, 2} . \\
\cdot\left(m_{N_{i, l}, 1}^{2}+m_{N_{i, l}, 2}^{2}+\left(m_{N_{i, l}, 1}+m_{N_{i, l}, 2}\right)^{2}\right)+\ldots+\left(1-\gamma_{N_{i, l}, 1}\right) \\
\cdot\left(1-\gamma_{N_{i, l}, 2}\right) \cdots\left(1-\gamma_{N_{i, l}, l-1}\right) \gamma_{N_{i, l}, l} \\
\cdot\left(m_{N_{i, l}, 1}^{2}+\ldots+m_{N_{i, l}, l}^{2}+\left(m_{N_{i, l}, 1}+\ldots+m_{N_{i, l}, l}\right)^{2}\right)
\end{array}
$$

em que $1 \leqslant l \leqslant r$.

Então

$$
E\left[W_{N_{., l}}^{2} 1_{A}\right]=\sum_{i=1}^{m} q_{i} E\left[W_{N_{i, l}}^{2} 1_{A}\right]
$$

e

$$
E\left[W_{N}^{2} 1_{A}\right]=\sum_{l=1}^{r} \sum_{N \in \mathcal{M}^{*}} \pi_{N}^{a} E\left[W_{N_{,, l}}^{2} 1_{A}\right]
$$

Os momentos condicionais são

$$
E\left(W_{N} \mid A\right)=\frac{E\left[W 1_{A}\right]}{P(A)} \quad \text { e } \quad E\left(W^{2} \mid A\right)=\frac{E\left[W^{2} 1_{A}\right]}{P(A)},
$$

para $P(A)$ em (3.45).

A variância condicional é dada por:

$$
\operatorname{Var}(W \mid A) \equiv E\left(W^{2} \mid A\right)-(E(W \mid A))^{2}
$$

Como indicado no Capítulo 2, usaremos aqui também as transformadas de Laplace para obter as soluções numéricas de $P(W ; A)$.

Assim desenvolvemos a extensão das medidas de desempenho aproximadas desenvolvidas por Ward Whitt em [15], para $m$ tipos de clientes. 
42 CAPÍTULO 3. CENTRAL DE ATENDIMENTO COM MAIS DE UM TIPO DE CLIENTE. 


\section{Capítulo 4}

\section{Casos particulares}

\subsection{Introdução}

Vamos apresentar dois casos particulares: $M / G^{i} / 1 / 1+G^{i}$, com $i=2$ e $M / G^{i} / 2 / 3+G^{i}$, com $i=2$.

Para o caso particular do modelo $M / G^{i} / 1 / 1+G^{i}, \operatorname{com} i=2$, serão apresentados quatro cenários.

Existem várias alternativas de escolha de distribuições e parâmetros, optamos por estudar os cenários abaixo:

Cenário 1: Clientes tipo 1 com distribuição de serviço e paciência Exponencial com taxa 0,5; e clientes tipo 2 com distribuição de serviço e paciência $\operatorname{Erlang}(2,2)$ com taxa 1.

Cenário 2: Clientes tipo 1 com distribuição de serviço e paciência Exponencial com taxa 2; e clientes tipo 2 com distribuição de serviço e paciência $\operatorname{Erlang}(1,1)$ com taxa 1.

Cenário 3: Clientes tipo 1 com distribuição de serviço e paciência $L N(1,4)$; e clientes tipo 2 com distribuição de serviço e paciência Exponencial com taxa 3.

Cenário 4: Clientes tipo 1 com distribuição de serviço e paciência $L N(1,4)$; e clientes tipo 2 com distribuição de serviço e paciência $\operatorname{Erlang}(1,1)$ com taxa 1.

Para o caso particular do modelo $M / G^{i} / 2 / 3+G^{i}$, com $i=2$, vamos apresentar um cenário:

Cenário 1: Clientes tipo 1 com distribuição de serviço e paciência Exponencial com taxa 0,5; e clientes tipo 2 com distribuição de serviço e paciência Exponencial com taxa 1.

Cenário 2: Clientes tipo 1 com distribuição de serviço e paciência Exponencial com taxa 2; e clientes tipo 2 com distribuição de serviço e paciência Erlang com taxa 1. 
Para fazer a aproximação definida no Capítulo 3, vamos efetuar os cálculos com o modelo $M / M^{i} / 1 / 1+M^{i}(n)$. Com os prâmetros escolhidos, descrevemos as equações de balanço, as distribuições estacionárias e as medidas de desempenho aproximadas desenvolvidas no Capítulo 3. Para o modelo $M / M^{i} / 2 / 3+M^{i}(n)$, efetuamos alguns exemplos e os cálculos são apresentados em anexo.

\subsection{Caso $M / M^{i} / 1 / 1+M^{i}(n), i=2$}

Nesta Seção vamos apresentar os cálculos do modelo de fila $M / M^{i} / 1 / 1+M^{i}(n)$, que possui 1 servidor (atendente) e 1 cliente pode aguardar atendimento, ou seja, a sala de espera possui tamanho 1. Como pode ser observado na Figura 4.1.

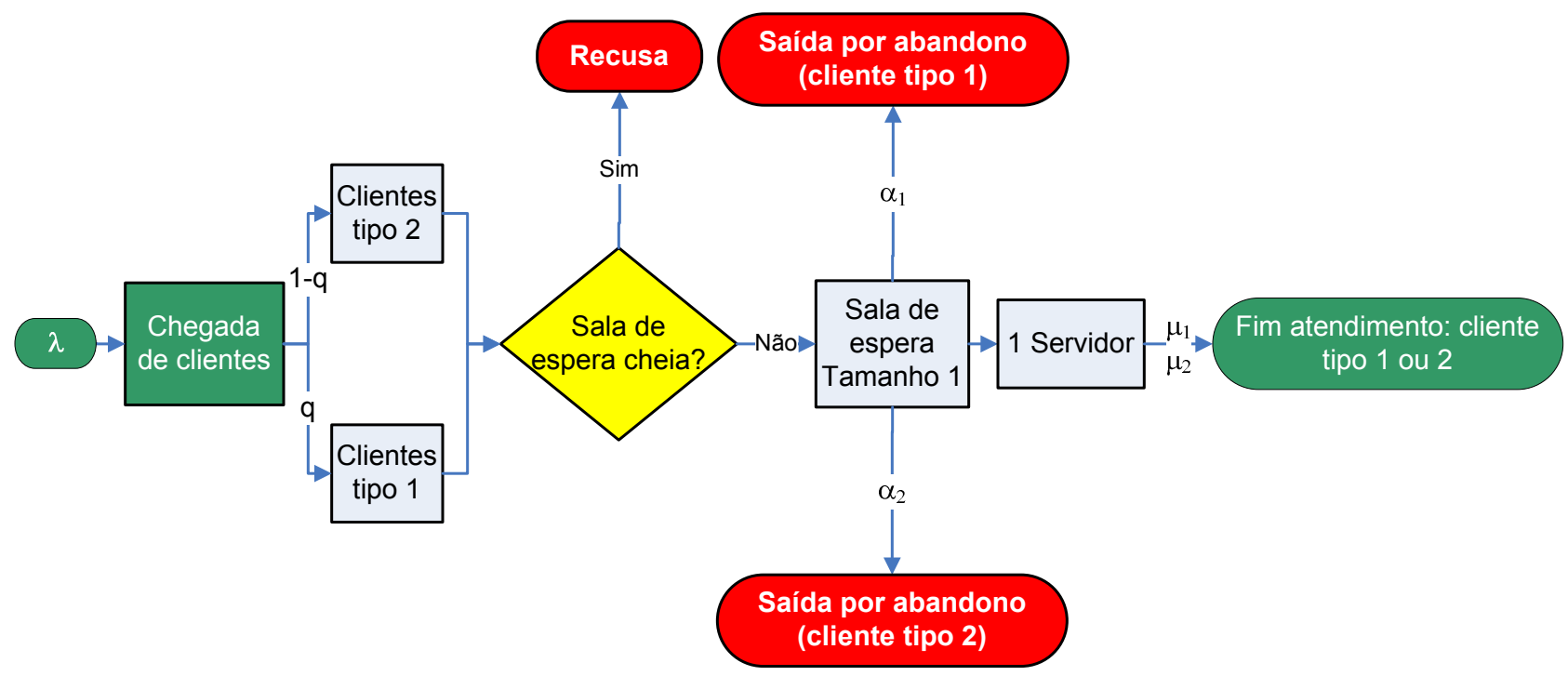

Figura 4.1: Modelo $M / M^{i} / 1 / 1+M^{i}(n)(i=2)$.

Os estados do sistema foram definidos como no Capítulo 3, por exemplo, o estado $(1,0 ; 0)$ representa um cliente do tipo 1 sendo atendido; $(0,1 ; 2)$ representa um cliente do tipo 2 sendo atendido e um cliente do tipo 2 aguardando na fila. O conjunto de todos os estados é dado por $\Omega$. Dessa forma, temos um processo Markoviano e podemos construir o fluixo e seus estados. 


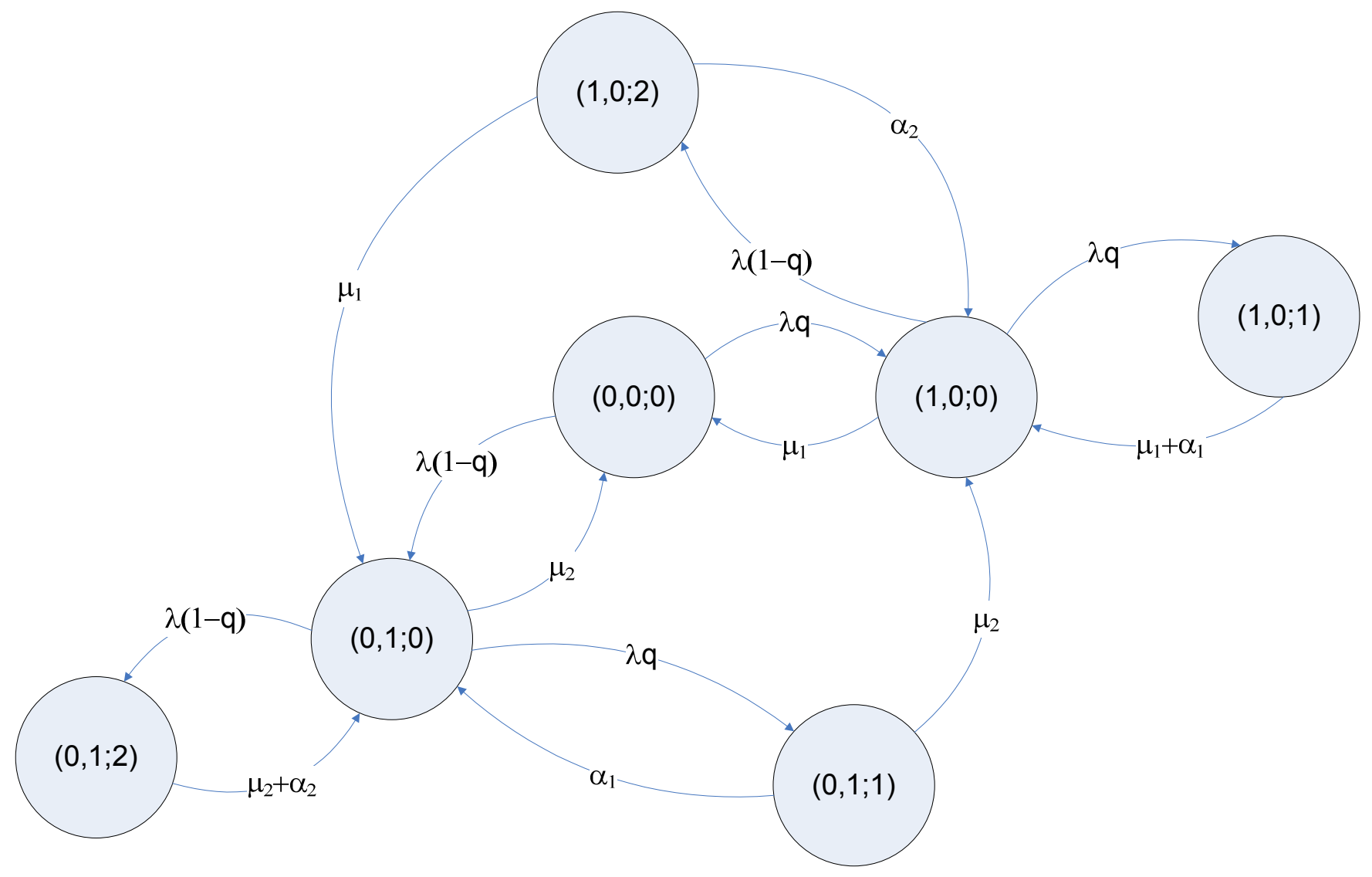

Figura 4.2: Fluxo do modelo $M / M^{i} / 1 / 1+M^{i}(n)(i=2)$.

Para definir as equações de balanço, vamos aplicar o conceito utilizado na Seção 3.2, desenvolvendo cada um dos casos. Considere $q=q_{1}$ e $(1-q)=q_{2}$.

Assim $\Omega=\{(0,0 ; 0),(1,0 ; 0),(0,1 ; 0),(1,0 ; 1),(1,0 ; 2),(0,1 ; 1),(0,1 ; 2)\}$, consideramos os seguintes casos:

- Caso 1: Através das equações 3.4, 3.5 e 3.6, vem:

$$
\begin{gathered}
N \in \mathcal{M}_{1}=\{(0,0 ; 0)\} ; \\
A_{i} \in \mathcal{A}_{i}=\{\emptyset\} ; \\
B_{i} \in \mathcal{B}_{i}=\{(1,0 ; 0),(0,1 ; 0)\} .
\end{gathered}
$$


A equação de balanço correspondente é definida como

$$
\pi_{(1,0 ; 0)} \mu_{1}+\pi_{(0,1 ; 0)} \mu_{2}=\pi_{(0,0 ; 0)} \lambda .
$$

- Caso 2: Através das equações 3.8, 3.9 e 3.10, vem:

$$
N \in \mathcal{M}_{2}=\{(1,0 ; 0),(0,1 ; 0)\}
$$

$\operatorname{Para} N=\{(1,0 ; 0)\}$,

$$
\begin{gathered}
C_{i}=\left\{\begin{array}{cc}
\{\emptyset\}, & i=1 ; \\
\{(0,0 ; 0)\}, & i=2 .
\end{array}\right. \\
D_{i}= \begin{cases}\{(0,1 ; 1)\}, & i=1 ; \\
\{(0,1 ; 2)\}, & i=2 .\end{cases}
\end{gathered}
$$

A equação de balanço correspondente é definida como

$$
\pi_{(0,0 ; 0)} \lambda q+\pi_{(0,1 ; 1)} \mu_{2}+\pi_{(1,0 ; 1)}\left(\mu_{1}+\alpha_{1,1}\right)+\pi_{(1,0 ; 2)} \alpha_{2,1}=\pi_{(1,0 ; 0)}\left(\mu_{1}+\lambda\right) .
$$

Para $N=\{(0,1 ; 0)\}$,

$$
\begin{gathered}
C_{i}=\left\{\begin{array}{cc}
\{(0,0 ; 0)\}, & i=1 ; \\
\{\emptyset\}, & i=2 .
\end{array}\right. \\
D_{i}=\left\{\begin{array}{cc}
\{(1,0 ; 1)\}, & i=1 ; \\
\{(1,0 ; 2)\}, & i=2 .
\end{array}\right.
\end{gathered}
$$

A equação de balanço correspondente é definida como

$$
\pi_{(0,0 ; 0)} \lambda(1-q)+\pi_{(1,0 ; 2)} \mu_{1}+\pi_{(0,1 ; 2)}\left(\mu_{2}+\alpha_{2,1}\right)+\pi_{(0,1 ; 1)} \alpha_{1,1}=\pi_{(0,1 ; 0)}\left(\mu_{2}+\lambda\right) .
$$

- Caso 3: Para $N$ definido por 3.13, vem:

$$
N \in \mathcal{M}_{3}=\{\emptyset\}
$$


Assim, para este caso particular, o Caso 3 não gera nenhuma equação de balanço.

- Caso 4: Através das equações 3.19, 3.20, 3.21, 3.22, temos:

$$
N \in \mathcal{M}_{4}=\{(1,0 ; 1),(1,0 ; 2),(0,1 ; 1),(0,1 ; 2)\}
$$

Para $N=\{(1,0 ; 1)\}$

$$
H_{i}=\left\{\begin{array}{cc}
\{(1,0 ; 0)\}, & i=1 \\
\{\emptyset\}, & i=2 .
\end{array}\right.
$$

A equação de balanço correspondente é definida como

$$
\pi_{(1,0 ; 0)} \lambda q=\pi_{(1,0 ; 1)}\left(\mu_{1}+\alpha_{1,1}\right)
$$

Para $N=\{(1,0 ; 2)\}$

$$
H_{i}=\left\{\begin{array}{cl}
\{\emptyset\}, & i=1 \\
\{(1,0 ; 0)\}, & i=2 .
\end{array}\right.
$$

A equação de balanço correspondente é definida como

$$
\pi_{(1,0 ; 0)} \lambda(1-q)=\pi_{(1,0 ; 2)}\left(\mu_{1}+\alpha_{2,1}\right) .
$$

Para $N=\{(0,1 ; 1)\}$,

$$
H_{i}=\left\{\begin{array}{cc}
\{(0,1 ; 0)\}, & i=1 \\
\{\emptyset\}, & i=2
\end{array}\right.
$$

A equação de balanço correspondente é definida como

$$
\pi_{(0,1 ; 0)} \lambda q=\pi_{(0,1 ; 1)}\left(\mu_{2}+\alpha_{1,1}\right)
$$

Para $N=\{(0,1 ; 2)\}$,

$$
H_{i}=\left\{\begin{array}{cl}
\{\emptyset\}, & i=1 ; \\
\{(0,1 ; 0)\}, & i=2 .
\end{array}\right.
$$


A equação de balanço correspondente é definida como

$$
\pi_{(0,1 ; 0)} \lambda(1-q)=\pi_{(0,1 ; 2)}\left(\mu_{2}+\alpha_{2,1}\right) .
$$

As equações de balanço acima foram definidas em função do fluxo entre os estados. São consideradas todas entradas e saídas dos estados. Assim equações são dadas por

$$
\begin{array}{r}
\pi_{(1,0 ; 0)} \mu_{1}+\pi_{(0,1 ; 0)} \mu_{2}=\pi_{(0,0 ; 0)} \lambda \\
\pi_{(0,0 ; 0)} \lambda q+\pi_{(0,1 ; 1)} \mu_{2}+\pi_{(1,0 ; 1)}\left(\mu_{1}+\alpha_{1,1}\right)+\pi_{(1,0 ; 2)} \alpha_{2,1}=\pi_{(1,0 ; 0)}\left(\mu_{1}+\lambda\right) \\
\pi_{(0,0 ; 0)} \lambda(1-q)+\pi_{(1,0 ; 2)} \mu_{1}+\pi_{(0,1 ; 2)}\left(\mu_{2}+\alpha_{2,1}\right)+\pi_{(0,1 ; 1)} \alpha_{1,1}=\pi_{(0,1 ; 0)}\left(\mu_{2}+\lambda\right) \\
\pi_{(1,0 ; 0)} \lambda q=\pi_{(1,0 ; 1)}\left(\mu_{1}+\alpha_{1,1}\right) \\
\pi_{(0,1 ; 0)} \lambda q=\pi_{(0,1 ; 1)}\left(\mu_{2}+\alpha_{1,1}\right) \\
\pi_{(1,0 ; 0)} \lambda(1-q)=\pi_{(1,0 ; 2)}\left(\mu_{1}+\alpha_{2,1}\right) \\
\pi_{(0,1 ; 0)} \lambda(1-q)=\pi_{(0,1 ; 2)}\left(\mu_{2}+\alpha_{2,1}\right),
\end{array}
$$

e considerando que a soma das probabilidades nos estados é 1,

$$
\pi_{(0,0 ; 0)}+\pi_{(1,0 ; 0)}+\pi_{(0,1 ; 0)}+\pi_{(1,0 ; 1)}+\pi_{(0,1 ; 1)}+\pi_{(1,0 ; 2)}+\pi_{(0,1 ; 2)}=1 .
$$

Então temos as equações da probabilidade estacionária dos estados $N \in \Omega$, que são dadas por

$$
\begin{gathered}
\pi_{(1,0 ; 1)}=\left(q ^ { 2 } \mu _ { 2 } \lambda ^ { 2 } \left(\alpha_{2,1} \mu_{1} \alpha_{1,1}+\alpha_{2,1} \mu_{2} \alpha_{1,1}+\lambda \alpha_{2,1}^{2}+\mu_{1} \lambda \mu_{2}+\alpha_{2,1} \lambda \mu_{2}+\alpha_{2,1}^{2} \mu_{2}+\right.\right. \\
\left.\left.+\mu_{1} \mu_{2}^{2}+\alpha_{2,1} \mu_{2}^{2}+\mu_{1} \alpha_{2,1} \mu_{2}+\mu_{1} \mu_{2} \alpha_{1,1}+\alpha_{2,1}^{2} \alpha_{1,1}+\mu_{1} \alpha_{2,1} \lambda\right)\right) / D \\
\pi_{(0,1 ; 1)}=\left(-\left(\mu_{2}+\alpha_{2,1}\right) \lambda^{2} q \mu_{1}\left(-\mu_{1} \alpha_{1,1}+q \mu_{1}^{2}+\mu_{1} q \alpha_{1,1}+q \mu_{1} \alpha_{2,1}+\alpha_{2,1} q \alpha_{1,1}-\right.\right. \\
\left.\left.-\mu_{1} \alpha_{2,1}-\alpha_{2,1} \alpha_{1,1}-\mu_{1}^{2}-\lambda \alpha_{1,1}-\lambda \mu_{1}+\lambda q \mu_{1}+\lambda q \alpha_{1,1}\right)\right) / D \\
\pi_{(1,0 ; 2)}=\left(-\left(\alpha_{2,1} \alpha_{1,1}+\lambda \mu_{2}+\mu_{2}^{2}+\alpha_{2,1} \mu_{2}+\mu_{2} \alpha_{1,1}+\right.\right. \\
\left.\left.\lambda \alpha_{2,1}\right) \lambda^{2} \mu_{2} q\left(-\alpha_{1,1}-\mu_{1}+q \mu_{1}+q \alpha_{1,1}\right)\right) / D \\
\pi_{(0,1 ; 2)}=\left(\left(-\mu_{1} \alpha_{1,1}+q \mu_{1}^{2}+\mu_{1} q \alpha_{1,1}+q \mu_{1} \alpha_{2,1}+\alpha_{2,1} q \alpha_{1,1}-\right.\right. \\
\left.-\mu_{1} \alpha_{2,1}-\alpha_{2,1} \alpha_{1,1}-\mu_{1}^{2}-\lambda \alpha_{1,1}-\lambda \mu_{1}+\lambda q \mu_{1}+\lambda q \alpha_{1,1}\right) \\
\left.\mu_{1} \lambda^{2}\left(-\mu_{2}-\alpha_{1,1}+q \mu_{2}+q \alpha_{1,1}\right)\right) / D
\end{gathered}
$$




$$
\begin{array}{r}
\pi_{(0,0 ; 0)}=\left(\mu _ { 1 } \mu _ { 2 } \left(\alpha_{2,1} \alpha_{1,1}^{2} \mu_{1}+\mu_{2} \alpha_{1,1}^{2} \mu_{1}+\mu_{2} \alpha_{1,1}^{2} \alpha_{2,1}+\alpha_{2,1}^{2} \mu_{2} \alpha_{1,1}+\right.\right. \\
+\mu_{1} \alpha_{2,1} \mu_{2}^{2}+\mu_{1} \lambda \mu_{2}^{2}+\mu_{1} \mu_{2}^{2} \alpha_{1,1}+\alpha_{2,1} \mu_{2}^{2} \alpha_{1,1}+\mu_{1} \lambda \mu_{2} \alpha_{1,1}+ \\
+2 \mu_{1} \alpha_{2,1} \mu_{2} \alpha_{1,1}+\alpha_{2,1} \lambda \mu_{2} \alpha_{1,1}+\mu_{1} \alpha_{2,1} \lambda \mu_{2}+\mu_{1} \alpha_{2,1} \lambda \alpha_{1,1}+ \\
+\mu_{2} \mu_{1}^{2} \alpha_{1,1}+\alpha_{2,1} \mu_{1}^{2} \alpha_{1,1}+\alpha_{2,1}^{2} \mu_{1} \alpha_{1,1}+\alpha_{2,1} \mu_{2} \mu_{1}^{2}+\alpha_{2,1}^{2} \alpha_{1,1}^{2}+ \\
+\mu_{2}^{2} \mu_{1}^{2}+q \alpha_{2,1}^{2} \lambda \alpha_{1,1}+q \alpha_{2,1}^{2} \lambda \mu_{1}-q \alpha_{1,1}^{2} \alpha_{2,1} \lambda-\lambda \mu_{2} \alpha_{1,1}^{2} q+ \\
+\lambda \mu_{2}^{2} \alpha_{1,1}+\lambda \mu_{2} \alpha_{1,1}^{2}+\alpha_{2,1} \lambda \alpha_{1,1}^{2}+\mu_{2} \alpha_{2,1}^{2} \mu_{1}-\mu_{2}^{2} \lambda q \mu_{1}- \\
\left.\left.-\mu_{2}^{2} \lambda q \alpha_{1,1}+q \mu_{2} \lambda \mu_{1}^{2}+q \alpha_{2,1} \lambda \mu_{1}^{2}\right)\right) / D
\end{array}
$$

$$
\begin{array}{r}
\pi_{(0,1 ; 0)}=\left(-\lambda\left(\mu_{2}+\alpha_{1,1}\right)\left(\mu_{2}+\alpha_{2,1}\right) \mu_{1}\left(-\mu_{1} \alpha_{1,1}+q \mu_{1}^{2}+\mu_{1} q \alpha_{1,1}+\right.\right. \\
\left.\left.q \mu_{1} \alpha_{2,1}+\alpha_{2,1} q \alpha_{1,1}-\mu_{1} \alpha_{2,1}-\alpha_{2,1} \alpha_{1,1}-\mu_{1}^{2}-\lambda \alpha_{1,1}-\lambda \mu_{1}+\lambda q \mu_{1}+\lambda q \alpha_{1,1}\right)\right) / D
\end{array}
$$

$$
\begin{array}{r}
\pi_{(1,0 ; 0)}=\left(\lambda \mu _ { 2 } q \left(\alpha_{2,1} \mu_{1}^{2} \alpha_{1,1}+2 \mu_{1} \alpha_{2,1} \mu_{2} \alpha_{1,1}+\alpha_{2,1}^{2} \lambda \mu_{1}+\mu_{2} \lambda \mu_{1}^{2}+\right.\right. \\
\mu_{1} \alpha_{2,1} \lambda \mu_{2}+\mu_{2} \alpha_{2,1}^{2} \mu_{1}+\mu_{2}^{2} \mu_{1}^{2}+\mu_{1} \alpha_{2,1} \mu_{2}^{2}+\alpha_{2,1} \mu_{2} \mu_{1}^{2}+ \\
\mu_{2} \mu_{1}^{2} \alpha_{1,1}+\alpha_{2,1}^{2} \mu_{1} \alpha_{1,1}+\alpha_{2,1} \lambda \mu_{1}^{2}+\alpha_{2,1} \alpha_{1,1}^{2} \mu_{1}+ \\
\mu_{2} \alpha_{1,1}^{2} \alpha_{2,1}+\alpha_{2,1}^{2} \lambda \alpha_{1,1}+\mu_{1} \lambda \mu_{2} \alpha_{1,1}+\alpha_{2,1} \lambda \mu_{2} \alpha_{1,1}+ \\
\left.\left.\alpha_{2,1}^{2} \mu_{2} \alpha_{1,1}+\mu_{1} \mu_{2}^{2} \alpha_{1,1}+\alpha_{2,1} \mu_{2}^{2} \alpha_{1,1}+\mu_{2} \alpha_{1,1}^{2} \mu_{1}+\alpha_{2,1}^{2} \alpha_{1,1}^{2}+\mu_{1} \alpha_{2,1} \lambda \alpha_{1,1}\right)\right) / D
\end{array}
$$

onde $D$ é definido por:

$$
\begin{array}{r}
D=q^{2} \mu_{2} \lambda^{2}\left(\mu_{2}+\alpha_{2,1}\right)\left(\mu_{1}+\alpha_{2,1}\right)\left(\lambda+\mu_{2}+\alpha_{1,1}\right)-\left(\mu_{2}+\alpha_{2,1}\right) \\
\lambda^{2} q \mu_{1}\left(\mu_{1}+\alpha_{1,1}\right)\left(\mu_{1}+\lambda+\alpha_{2,1}\right)(-1+q)-\left(\mu_{2}+\alpha_{2,1}\right)\left(\lambda+\mu_{2}+\alpha_{1,1}\right) \\
\lambda^{2} \mu_{2} q\left(\mu_{1}+\alpha_{1,1}\right)(-1+q)+\left(\mu_{1}+\alpha_{1,1}\right)\left(\mu_{1}+\lambda \alpha_{2,1}\right)(-1+q)^{2} \mu_{1} \lambda^{2}\left(\mu_{2}+\right. \\
\left.+\alpha_{1,1}\right)+\mu_{1} \mu_{2}\left(\mu_{2}+\alpha_{2,1}\right)\left(\mu_{1}+\alpha_{1,1}\right)\left(\mu_{1} \mu_{2}+\lambda q \mu_{1}+\mu_{1} \alpha_{1,1}+\right. \\
\left.+\lambda \mu_{2}+\alpha_{2,1} \mu_{2}-\lambda q \mu_{2}+\lambda \alpha_{1,1}+\alpha_{2,1} \alpha_{1,1}-\lambda q \alpha_{1,1}+\lambda q \alpha_{2,1}\right)- \\
-\lambda\left(\mu_{2}+\alpha_{1,1}\right)\left(\mu_{2}+\alpha_{2,1}\right) \mu_{1}\left(\mu_{1}+\alpha_{1,1}\right)\left(\mu_{1}+\lambda+\alpha_{2,1}\right)(-1+q)+ \\
+\lambda \mu_{2} q\left(\mu_{1}+\alpha_{2,1}\right)\left(\mu_{1}+\alpha_{1,1}\right)\left(\mu_{2}+\alpha_{2,1}\right)\left(\lambda+\mu_{2}+\alpha_{1,1}\right) .
\end{array}
$$




\subsubsection{Medidas de desempenho caso $M / M^{i} / 1 / 1+M^{i}(n), i=2$}

As medidas de desempenho dadas por Whitt em [15] e adequadas para $m$ tipos de clientes na Seção A.1.3, são agora apresentadas para o caso $M / M^{i} / 1 / 1+M^{i}(n), \operatorname{com} i=2$.

Todas as equações a seguir já foram definidas na Seção A.1.3, aqui vamos aplicá-las para o caso particular $M / M^{i} / 1 / 1+M^{i}(n), \operatorname{com} i=2$.

A probabilidade do sistema estar completo indica a probabilidade de uma chegada ser recusada, definida em 3.29, é

$$
P(\text { Recusa })=\pi_{(0,1 ; 1)}+\pi_{(1,0 ; 1)}+\pi_{(1,0 ; 2)}+\pi_{(0,1 ; 2)}
$$

Seja $N \in \Omega^{*}=\Omega-\mathcal{M}_{4}$, então $\pi_{N}$, definida em 3.30, é a probabilidade estacionária do sistema no estado $N$ (com $m$ tipos de clientes),

$$
\pi_{N}^{a}= \begin{cases}\frac{\pi_{(0,0 ; 0)}}{1-P(\text { Recusa })}, & \text { para } N=(0,0 ; 0) \\ \frac{\pi_{(1,0 ; 0)}}{1-P(\text { Recusa })}, & \text { para } N=(1,0 ; 0) \\ \frac{\pi_{(0,1 ; 0)}}{1-P(\text { Recusa })}, & \text { para } N=(0,1 ; 0) .\end{cases}
$$

A probabilidade do cliente entrar no sistema e ser atendido no mesmo instante, definida em 3.31 , é

$$
P(\text { Atendimento Imediato })=\pi_{(0,0 ; 0)}^{a}
$$


A probabilidade do cliente do tipo $i$ abandonar na posição 1 (contada à partir do fim da fila), definida em 3.32, é

$$
\gamma_{N_{i, 1}, 1} \approx \begin{cases}\frac{\alpha_{1,1}}{\left(\mu_{1} q_{1}+\mu_{2} q_{2}\right)+\alpha_{1,1}}, & \text { para } N=(1,0 ; 1) \\ \frac{\alpha_{2,1}}{\left(\mu_{1} q_{1}+\mu_{2} q_{2}\right)+\alpha_{2,1}}, & \text { para } N=(1,0 ; 2) \\ \frac{\alpha_{1,1}}{\left(\mu_{1} q_{1}+\mu_{2} q_{2}\right)+\alpha_{1,1}}, & \text { para } N=(0,1 ; 1) \\ \frac{\alpha_{2,1}}{\left(\mu_{1} q_{1}+\mu_{2} q_{2}\right)+\alpha_{2,1}}, & \text { para } N=(0,1 ; 2) .\end{cases}
$$

em que $i=\{1,2\}$.

Logo a probabilidade do cliente no estado $N_{i, 1}$ não abandonar o sistema até ser servido, definida em 3.33, é:

$$
\Gamma_{N_{i, 1}} \approx \begin{cases}1-\frac{\alpha_{1,1}}{\left(\mu_{1} q_{1}+\mu_{2} q_{2}\right)+\alpha_{1,1}}, & \text { para } N=(1,0 ; 1) ; \\ 1-\frac{\alpha_{2,1}}{\left(\mu_{1} q_{1}+\mu_{2} q_{2}\right)+\alpha_{2,1}}, & \text { para } N=(1,0 ; 2) ; \\ 1-\frac{\alpha_{1,1}}{\left(\mu_{1} q_{1}+\mu_{2} q_{2}\right)+\alpha_{1,1}}, & \text { para } N=(0,1 ; 1) ; \\ 1-\frac{\alpha_{2,1}}{\left(\mu_{1} q_{1}+\mu_{2} q_{2}\right)+\alpha_{2,1}}, & \text { para } N=(0,1 ; 2) .\end{cases}
$$

E a probabilidade do cliente que chegou na fila na posição 1 não abandonar até ser servido (independente de seu tipo), definida em 3.34, é 


$$
\Gamma_{N_{\text {., }}} \approx \begin{cases}q_{1}\left(1-\frac{\alpha_{1,1}}{\left(\mu_{1} q_{1}+\mu_{2} q_{2}\right)+\alpha_{1,1}}\right), & \text { para } N=(1,0 ; 1) ; \\ q_{2}\left(1-\frac{\alpha_{2,1}}{\left(\mu_{1} q_{1}+\mu_{2} q_{2}\right)+\alpha_{2,1}}\right), & \text { para } N=(1,0 ; 2) ; \\ q_{1}\left(1-\frac{\alpha_{1,1}}{\left(\mu_{1} q_{1}+\mu_{2} q_{2}\right)+\alpha_{1,1}}\right), & \text { para } N=(0,1 ; 1) ; \\ q_{2}\left(1-\frac{\alpha_{2,1}}{\left(\mu_{1} q_{1}+\mu_{2} q_{2}\right)+\alpha_{2,1}}\right), & \text { para } N=(0,1 ; 2) .\end{cases}
$$

Definido em 3.35, a probabilidade de um cliente que entrou no sistema ser atendido é dada por:

$$
\begin{aligned}
P(S)=\pi_{(0,0 ; 0)}^{a}+ & \left(\pi_{(1,0 ; 0)}^{a} q_{1}\left(1-\frac{\alpha_{1,1}}{\left(\mu_{1} q_{1}+\mu_{2} q_{2}\right)+\alpha_{1,1}}\right)+\right. \\
& +\pi_{(1,0 ; 0)}^{a} q_{2}\left(1-\frac{\alpha_{2,1}}{\left(\mu_{1} q_{1}+\mu_{2} q_{2}\right)+\alpha_{2,1}}\right)+ \\
& +\pi_{(0,1 ; 0)}^{a} q_{1}\left(1-\frac{\alpha_{1,1}}{\left(\mu_{1} q_{1}+\mu_{2} q_{2}\right)+\alpha_{1,1}}\right)+ \\
& +\pi_{(0,1 ; 0)}^{a} q_{2}\left(1-\frac{\alpha_{2,1}}{\left(\mu_{1} q_{1}+\mu_{2} q_{2}\right)+\alpha_{2,1}}\right) .
\end{aligned}
$$

O tempo médio entre o $(j-1)$-ésimo e $j$-ésimo eventos de partida, definido em 3.37, é dado por

$$
m_{N_{i, 1}, 1} \approx \begin{cases}\frac{1}{\left(\mu_{1} q_{1}+\mu_{2} q_{2}\right)+\alpha_{1,1}}, & \text { para } N=(1,0 ; 1) ; \\ \frac{1}{\left(\mu_{1} q_{1}+\mu_{2} q_{2}\right)+\alpha_{2,1}}, & \text { para } N=(1,0 ; 2) ; \\ \frac{1}{\left(\mu_{1} q_{1}+\mu_{2} q_{2}\right)+\alpha_{1,1}}, & \text { para } N=(0,1 ; 1) ; \\ \frac{1}{\left(\mu_{1} q_{1}+\mu_{2} q_{2}\right)+\alpha_{2,1}}, & \text { para } N=(0,1 ; 2) .\end{cases}
$$

em que $i=\{1,2\}$. 
Então, como definido em 3.38:

$$
m_{N_{., 1}, 1} \approx \begin{cases}q_{1} \frac{1}{\left(\mu_{1} q_{1}+\mu_{2} q_{2}\right)+\alpha_{1,1}}, & \text { para } N=(1,0 ; 1) ; \\ q_{2} \frac{1}{\left(\mu_{1} q_{1}+\mu_{2} q_{2}\right)+\alpha_{2,1}}, & \text { para } N=(1,0 ; 2) ; \\ q_{1} \frac{1}{\left(\mu_{1} q_{1}+\mu_{2} q_{2}\right)+\alpha_{1,1}}, & \text { para } N=(0,1 ; 1) ; \\ q_{2} \frac{1}{\left(\mu_{1} q_{1}+\mu_{2} q_{2}\right)+\alpha_{2,1}}, & \text { para } N=(0,1 ; 2) .\end{cases}
$$

Para $E[W ; S]$ definido em 3.40, temos:

$$
\begin{aligned}
E[W ; S]= & \pi_{(1,0 ; 0)}^{a} q_{1}\left(1-\frac{\alpha_{1,1}}{\left(\mu_{1} q_{1}+\mu_{2} q_{2}\right)+\alpha_{1,1}}\right)\left(\frac{1}{\left(\mu_{1} q_{1}+\mu_{2} q_{2}\right)+\alpha_{1,1}}\right)+ \\
& +\pi_{(1,0 ; 0)}^{a} q_{2}\left(1-\frac{\alpha_{2,1}}{\left(\mu_{1} q_{1}+\mu_{2} q_{2}\right)+\alpha_{2,1}}\right)\left(\frac{1}{\left(\mu_{1} q_{1}+\mu_{2} q_{2}\right)+\alpha_{2,1}}\right)+ \\
& +\pi_{(0,1 ; 0)}^{a} q_{1}\left(1-\frac{\alpha_{1,1}}{\left(\mu_{1} q_{1}+\mu_{2} q_{2}\right)+\alpha_{1,1}}\right)\left(\frac{1}{\left(\mu_{1} q_{1}+\mu_{2} q_{2}\right)+\alpha_{1,1}}\right)+ \\
& +\pi_{(0,1 ; 0)}^{a} q_{2}\left(1-\frac{\alpha_{2,1}}{\left(\mu_{1} q_{1}+\mu_{2} q_{2}\right)+\alpha_{2,1}}\right)\left(\frac{1}{\left(\mu_{1} q_{1}+\mu_{2} q_{2}\right)+\alpha_{2,1}}\right),
\end{aligned}
$$

e também:

$$
\begin{aligned}
E\left[W^{2} ; S\right]= & \pi_{(1,0 ; 0)}^{a} q_{1}\left(1-\frac{\alpha_{1,1}}{\left(\mu_{1} q_{1}+\mu_{2} q_{2}\right)+\alpha_{1,1}}\right) \cdot 2 \cdot\left(\frac{1}{\left(\mu_{1} q_{1}+\mu_{2} q_{2}\right)+\alpha_{1,1}}\right)^{2}+ \\
& +\pi_{(1,0 ; 0)}^{a} q_{2}\left(1-\frac{\alpha_{2,1}}{\left(\mu_{1} q_{1}+\mu_{2} q_{2}\right)+\alpha_{2,1}}\right) \cdot 2 \cdot\left(\frac{1}{\left(\mu_{1} q_{1}+\mu_{2} q_{2}\right)+\alpha_{2,1}}\right)^{2}+ \\
& +\pi_{(0,1 ; 0)}^{a} q_{1}\left(1-\frac{\alpha_{1,1}}{\left(\mu_{1} q_{1}+\mu_{2} q_{2}\right)+\alpha_{1,1}}\right) \cdot 2 \cdot\left(\frac{1}{\left(\mu_{1} q_{1}+\mu_{2} q_{2}\right)+\alpha_{1,1}}\right)^{2}+ \\
& +\pi_{(0,1 ; 0)}^{a} q_{2}\left(1-\frac{\alpha_{2,1}}{\left(\mu_{1} q_{1}+\mu_{2} q_{2}\right)+\alpha_{2,1}}\right) \cdot 2 \cdot\left(\frac{1}{\left(\mu_{1} q_{1}+\mu_{2} q_{2}\right)+\alpha_{2,1}}\right)^{2} .
\end{aligned}
$$


Para as esperanças definidas em 3.48 e 3.51, temos:

$$
\begin{aligned}
E\left[W_{N} 1_{A}\right]= & \pi_{(1,0 ; 0)}^{a} q_{1}\left(\frac{\alpha_{1,1}}{\left(\mu_{1} q_{1}+\mu_{2} q_{2}\right)+\alpha_{1,1}}\right)\left(\frac{1}{\left(\mu_{1} q_{1}+\mu_{2} q_{2}\right)+\alpha_{1,1}}\right)+ \\
& +\pi_{(1,0 ; 0)}^{a} q_{2}\left(\frac{\alpha_{2,1}}{\left(\mu_{1} q_{1}+\mu_{2} q_{2}\right)+\alpha_{2,1}}\right)\left(\frac{1}{\left(\mu_{1} q_{1}+\mu_{2} q_{2}\right)+\alpha_{2,1}}\right)+ \\
& +\pi_{(0,1 ; 0)}^{a} q_{1}\left(\frac{\alpha_{1,1}}{\left(\mu_{1} q_{1}+\mu_{2} q_{2}\right)+\alpha_{1,1}}\right)\left(\frac{1}{\left(\mu_{1} q_{1}+\mu_{2} q_{2}\right)+\alpha_{1,1}}\right)+ \\
& +\pi_{(0,1 ; 0)}^{a} q_{2}\left(\frac{\alpha_{2,1}}{\left(\mu_{1} q_{1}+\mu_{2} q_{2}\right)+\alpha_{2,1}}\right)\left(\frac{1}{\left(\mu_{1} q_{1}+\mu_{2} q_{2}\right)+\alpha_{2,1}}\right),
\end{aligned}
$$

$\mathrm{e}$

$$
\begin{aligned}
E\left[W_{N}^{2} 1_{A}\right]= & \pi_{(1,0 ; 0)}^{a} q_{1}\left(\frac{\alpha_{1,1}}{\left(\mu_{1} q_{1}+\mu_{2} q_{2}\right)+\alpha_{1,1}}\right) \cdot 2 \cdot\left(\frac{1}{\left(\mu_{1} q_{1}+\mu_{2} q_{2}\right)+\alpha_{1,1}}\right)^{2}+ \\
& +\pi_{(1,0 ; 0)}^{a} q_{2}\left(\frac{\alpha_{2,1}}{\left(\mu_{1} q_{1}+\mu_{2} q_{2}\right)+\alpha_{2,1}}\right) \cdot 2 \cdot\left(\frac{1}{\left(\mu_{1} q_{1}+\mu_{2} q_{2}\right)+\alpha_{2,1}}\right)^{2}+ \\
& +\pi_{(0,1 ; 0)}^{a} q_{1}\left(\frac{\alpha_{1,1}}{\left(\mu_{1} q_{1}+\mu_{2} q_{2}\right)+\alpha_{1,1}}\right) \cdot 2 \cdot\left(\frac{1}{\left(\mu_{1} q_{1}+\mu_{2} q_{2}\right)+\alpha_{1,1}}\right)^{2}+ \\
& +\pi_{(0,1 ; 0)}^{a} q_{2}\left(\frac{\alpha_{2,1}}{\left(\mu_{1} q_{1}+\mu_{2} q_{2}\right)+\alpha_{2,1}}\right) \cdot 2 \cdot\left(\frac{1}{\left(\mu_{1} q_{1}+\mu_{2} q_{2}\right)+\alpha_{2,1}}\right)^{2} .
\end{aligned}
$$

Através das expressões acima e com auxilio da tranformada de Laplace, obtemos os resultados numéricos desejados que serão apresentados no final do capítulo.

\subsubsection{Validação}

Nesta seção validamos os algoritmos desenvolvidos em $R$, utilizados na simulação e no cálculo aproximado das medidas de interesse dos modelos com dois tipos de clientes. Para verificar a consistência dos algoritmos (simulação e aproximação) para 2 tipos de clientes, impomos $q_{1}=0$ e checamos se as soluções são próximas do que havíamos desenvolvido para apenas um tipo de cliente. Ver Tabelas 4.1 e 4.2. Os modelos estudados foram os seguintes:

(1) Simulação e Aproximação para 1 tipo de cliente: $M / M / 1 / 1+M$, serviço e paciência seguem distribuição Exponencial com taxa 1, 1 servidor, 1 espaço na sala de espera, taxa de chegada $\lambda=1$, utilizamos 30 réplicas com 1000 chegadas de clientes cada.

(2) Simulação e Aproximação para 1 tipo de cliente: $M / M^{i} / 1 / 1+M^{i}(i=2)$, sendo a probabilidade para cliente tipo $1 q_{1}=0$, serviço e paciência seguem distribuição Exponencial com taxa 1, 1 servidor, 1 espaço na sala de espera, taxa de chegada $\lambda=1$, utilizamos 30 réplicas com 1000 chegadas de clientes cada. 
Pode ser observado que as variações estão bastante pequenas. O maior percentual de variação está na $P(A)$ da simulação, com uma variação de $2,9 \%$. Desta forma, concluímos que nossos algoritmos de simulação e de aproximação, para dois tipos de clientes, estão validados. 


\section{Simulação: $M / M^{i} / 1 / 1+M^{i}, i=2$}

\begin{tabular}{|c|c|c|c|c|}
\hline $\begin{array}{c}\text { Medidas } \\
\text { de } \\
\text { Desempenho }\end{array}$ & $\begin{array}{c}\text { Modelo } \\
\text { Whitt }\end{array}$ & $\begin{array}{c}\text { Modelo } \\
\text { Gabriela } \\
q_{1}=1\end{array}$ & Variação & $\begin{array}{c}\text { Percentual } \\
\text { de } \\
\text { Variação }\end{array}$ \\
\hline$P(W=0)$ & 0,50 & 0,50 & 0,00 & $-0,1 \%$ \\
\hline$P(A)$ & 0,26 & 0,25 & 0,01 & $2,9 \%$ \\
\hline $\mathrm{E}[\mathrm{Q}]$ & 0,21 & 0,21 & 0,00 & $0,0 \%$ \\
\hline $\operatorname{Var}(\mathrm{Q})$ & 0,17 & 0,17 & 0,00 & $0,0 \%$ \\
\hline $\mathrm{E}[\mathrm{N}]$ & 1,00 & 1,00 & 0,00 & $0,0 \%$ \\
\hline $\mathrm{E}[\mathrm{W} \mid \mathrm{S}]$ & 0,17 & 0,17 & 0,00 & $0,1 \%$ \\
\hline $\operatorname{Var}(\mathrm{W} \mid \mathrm{S})$ & 0,14 & 0,14 & 0,00 & $0,5 \%$ \\
\hline$E[W \mid A]$ & 0,50 & 0,50 & 0,00 & $0,0 \%$ \\
\hline $\operatorname{Var}(\mathrm{W} \mid \mathrm{A})$ & 0,25 & 0,25 & 0,00 & $0,0 \%$ \\
\hline$P(W \leq 0.1 \mid S)$ & 0,73 & 0,73 & 0,00 & $0,0 \%$ \\
\hline$P(W \leq 0.1 \mid A)$ & 0,18 & 0,18 & 0,00 & $0,2 \%$ \\
\hline $\mathrm{P}(\mathrm{W} \leq 0.2 \mid \mathrm{S})$ & 0,78 & 0,78 & 0,00 & $0,0 \%$ \\
\hline$P(W \leq 0.2 \mid A)$ & 0,33 & 0,33 & 0,00 & $-0,1 \%$ \\
\hline $\begin{array}{l}\text { Parâmetros: } \\
s=1 \\
r=1 \\
\text { Chegadas: } \\
\text { Poisson } \lambda=1 \\
\text { início: } 1.000 \\
\text { número chegadas: } 50.000 \\
\text { número réplicas: } 30\end{array}$ & \begin{tabular}{|l} 
Serviços: \\
Exponencial(1) \\
Paciência: \\
Exponencial(1)
\end{tabular} & $\begin{array}{l}\mathbf{q}_{1}=\mathbf{1} \\
\text { Serviços: } \\
\text { Tipo 1: } \\
\quad \text { Exponencial(1) } \\
\text { Tipo 2: } \\
\quad \text { Exponencial(1) } \\
\text { Paciência: } \\
\text { Tipo 1: } \\
\text { Exponencial(1) } \\
\text { Tipo 2: } \\
\text { Exponencial(1) }\end{array}$ & & \\
\hline
\end{tabular}

Tabela 4.1: Validação da silmulação para filas com dois tipos de clientes. 


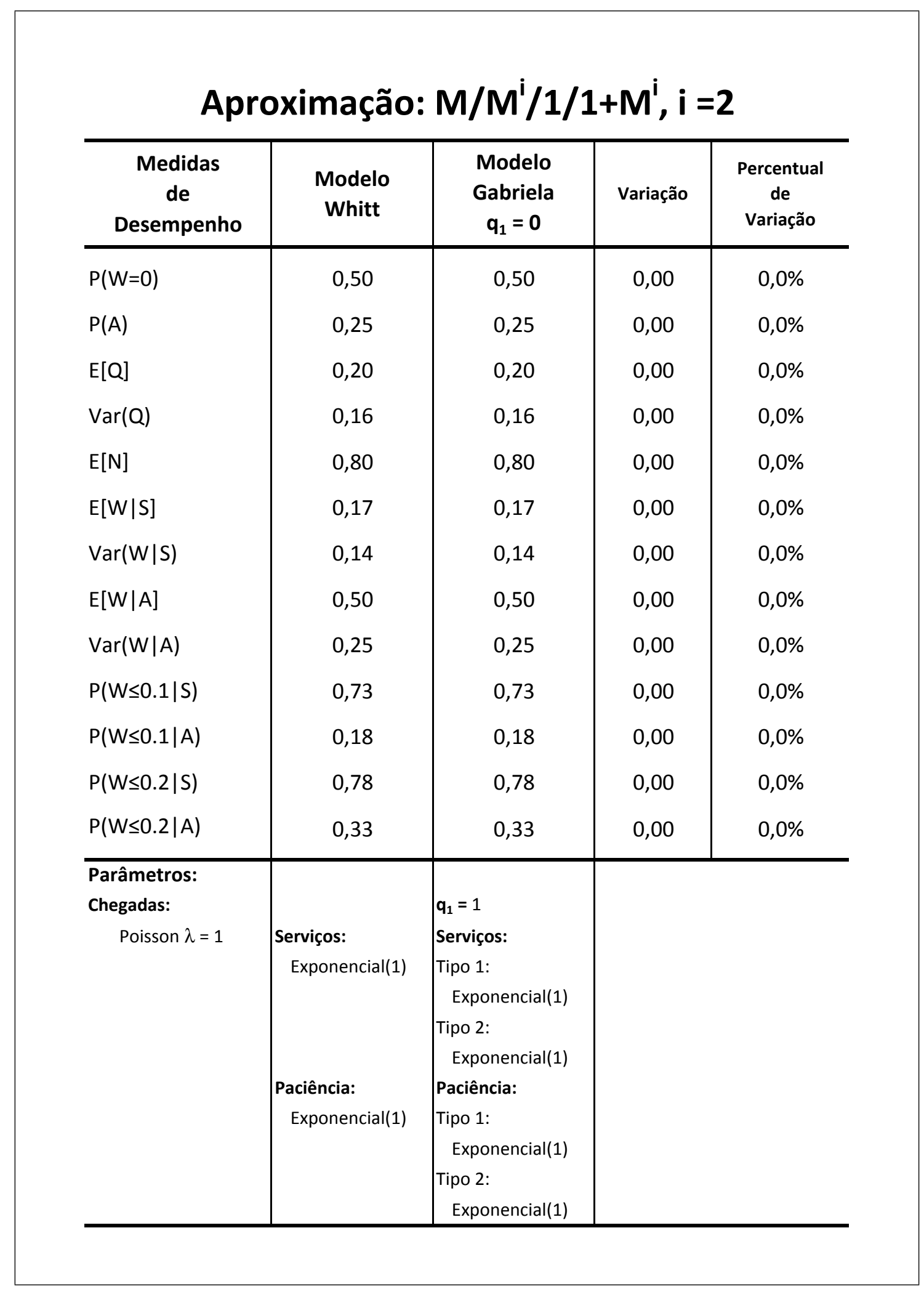

Tabela 4.2: Validação da aproximação para filas com dois tipos de clientes. 


\subsubsection{Resultados Numéricos e Análises}

Conforme descrito no início deste capítulo, nesta seção estudamos dois cenários de resultados numéricos para o caso particular $M / M^{i} / 1 / 1+M^{i}(n)(i=2)$.

Observando os dois cenários estudados, podemos verificar que seus resultados aproximados e simulados estão bastante próximos.

As tabelas 4.3 e 4.4 exibem a proximidade destes resultados. Assim concluímos que a aproximação proposta no Capítulo 3 é válida. 


\begin{tabular}{|c|c|c|c|c|}
\hline \multirow{3}{*}{$\begin{array}{l}\begin{array}{c}\text { Medidas } \\
\text { de } \\
\text { Desenpenho }\end{array} \\
\mathrm{P}(\mathrm{W}=0)\end{array}$} & \multicolumn{2}{|c|}{$M / G^{i} / 1 / 1+G^{i}-$ Cenário 1} & \multicolumn{2}{|c|}{$M / G^{i} / 1 / 1+G^{i}$ - Cenário 2} \\
\hline & Simulação & Aproximação & Simulação & Aproximação \\
\hline & 0,3638 & 0,3700 & 0,6060 & 0,6102 \\
\hline & $\pm 0,001$ & - & $\pm 0,001$ & - \\
\hline \multirow[t]{2}{*}{$P(A)$} & 0,3188 & 0,3164 & 0,2072 & 0,1873 \\
\hline & $\pm 0,0009$ & - & $\pm 0,0008$ & - \\
\hline \multirow[t]{2}{*}{$E[Q]$} & 0,3086 & 0,3410 & 0,1474 & 0,1135 \\
\hline & $\pm 0,0009$ & - & $\pm 0,0006$ & - \\
\hline \multirow[t]{2}{*}{$\operatorname{Var}(Q)$} & 0,2134 & 0,2247 & 0,1281 & 0,1006 \\
\hline & $\pm 0,0003$ & - & $\pm 0,0004$ & - \\
\hline \multirow[t]{2}{*}{$E[N]$} & 1,1255 & 1,0984 & 0,9352 & 0,5762 \\
\hline & $\pm 0,0013$ & - & $\pm 0,001$ & - \\
\hline \multirow[t]{2}{*}{$E[W \mid S]$} & 0,3794 & 0,3655 & 0,0761 & 0,0766 \\
\hline & $\pm 0,0016$ & - & $\pm 0,0006$ & - \\
\hline \multirow[t]{2}{*}{$\operatorname{Var}(\mathrm{W} \mid \mathrm{S})$} & 0,5013 & 0,4684 & 0,0464 & 0,0424 \\
\hline & $\pm 0,0055$ & - & $\pm 0,0006$ & - \\
\hline \multirow[t]{2}{*}{$E[W \mid A]$} & 0,8835 & 0,7349 & 0,3252 & 0,3037 \\
\hline & $\pm 0,0037$ & - & $\pm 0,0015$ & - \\
\hline \multirow[t]{2}{*}{$\operatorname{Var}(\mathrm{W} \mid \mathrm{A})$} & 0,7152 & 0,5889 & 0,1179 & 0,0894 \\
\hline & $\pm 0,0105$ & - & $\pm 0,0012$ & - \\
\hline \multirow[t]{2}{*}{$P(W \leq 0.1 \mid S)$} & 0,5856 & 0,5791 & 0,8297 & 0,7821 \\
\hline & $\pm 0,0013$ & - & $\pm 0,0008$ & - \\
\hline \multirow[t]{2}{*}{$P(W \leq 0.1 \mid A)$} & 0,0762 & 0,1160 & 0,2740 & 0,1634 \\
\hline & $\pm 0,0009$ & - & $\pm 0,0021$ & - \\
\hline \multirow[t]{2}{*}{$P(W \leq 0.2 \mid S)$} & 0,6337 & 0,6117 & 0,8763 & 0,8043 \\
\hline & $\pm 0,0012$ & - & $\pm 0,0008$ & - \\
\hline \multirow[t]{2}{*}{$\mathrm{P}(\mathrm{W} \leq 0.2 \mid \mathrm{A})$} & 0,1609 & 0,2152 & 0,4708 & 0,2785 \\
\hline & $\pm 0,0012$ & - & $\pm 0,0024$ & - \\
\hline Parâmetros: & Cenário 1 & & Cenário 2 & \\
\hline $\mathbf{s}=1$ & $q_{1}=0,7$ & & $q_{1}=0,7$ & \\
\hline$r=1$ & Serviços: & & Serviços: & \\
\hline Chegadas: Poisson $\lambda=1$ & Tipo 1: Exponencia & & Tipo 1: Exponencia & \\
\hline Tipos: $\mathrm{i}=2$ & Tipo 2: Erlang $(2,2)$ & & Tipo 2: $\operatorname{Erlang}(1,1)$ & \\
\hline início: 1.000 & Paciência: & & Paciência: & \\
\hline número hegadas: 50.000 & Tipo 1: Exponencia & & Tipo 1: Exponencia & \\
\hline número réplicas: 30 & Tipo 2: Erlang(2,2) & & Tipo 2: Erlang(1,1) & \\
\hline
\end{tabular}

Tabela 4.3: Resultados cenários 1 e 2, para o modelo $M / M^{i} / 1 / 1+M^{i}(n)(i=2)$. 


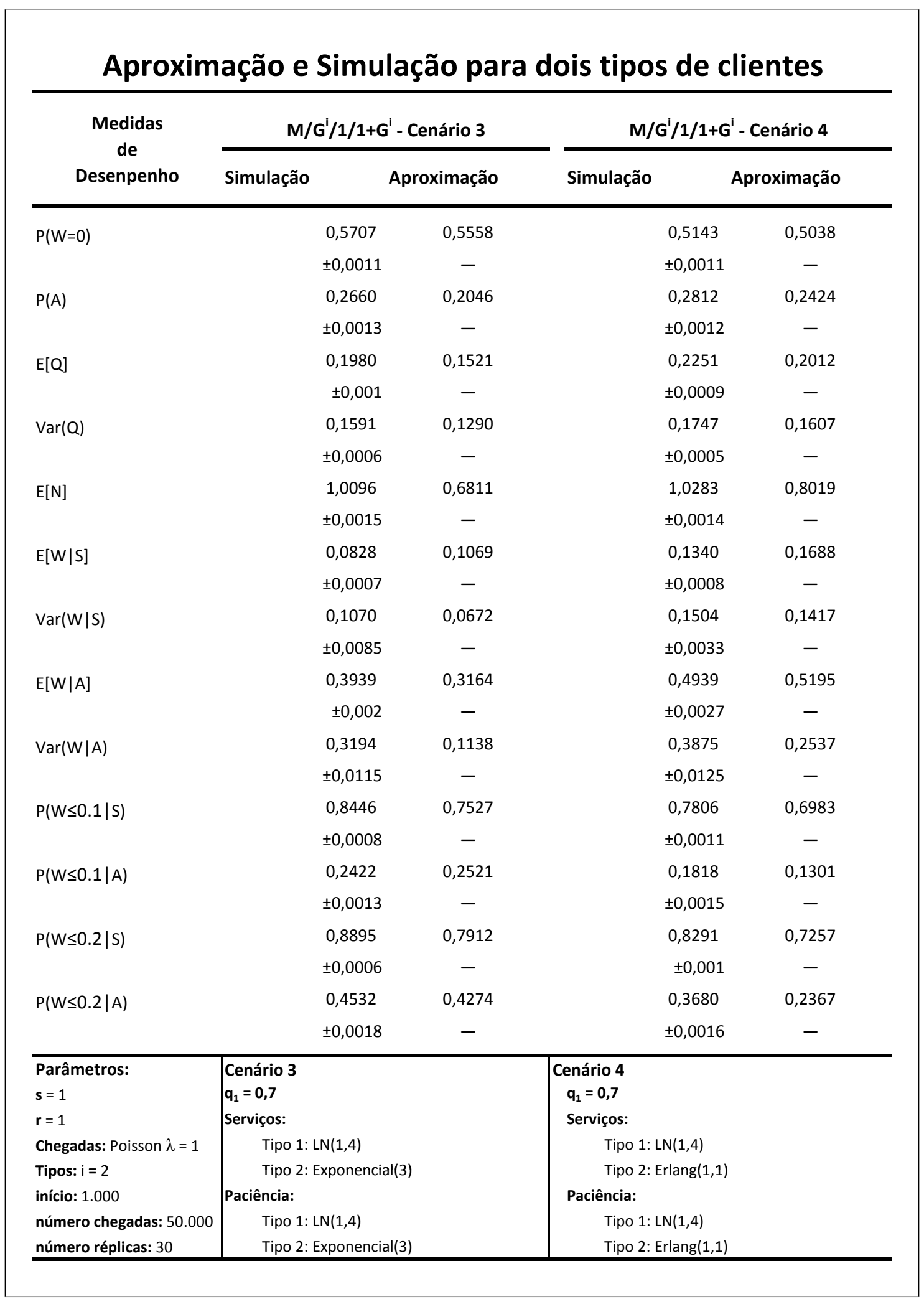

Tabela 4.4: Resultados cenários 3 e 4, para o modelo $M / M^{i} / 1 / 1+M^{i}(n)(i=2)$ 


\subsection{Caso $M / M^{i} / 2 / 3+M^{i}(n), i=2$}

Nesta Seção vamos apresentar os cálculos do modelo de fila $M / M^{i} / 2 / 3+M^{i}(n)$, que possui 2 servidores (atendentes) e 3 clientes podem aguardar atendimento, ou seja, a sala de espera possui tamanho 3. Como pode ser observado na Figura 4.3.

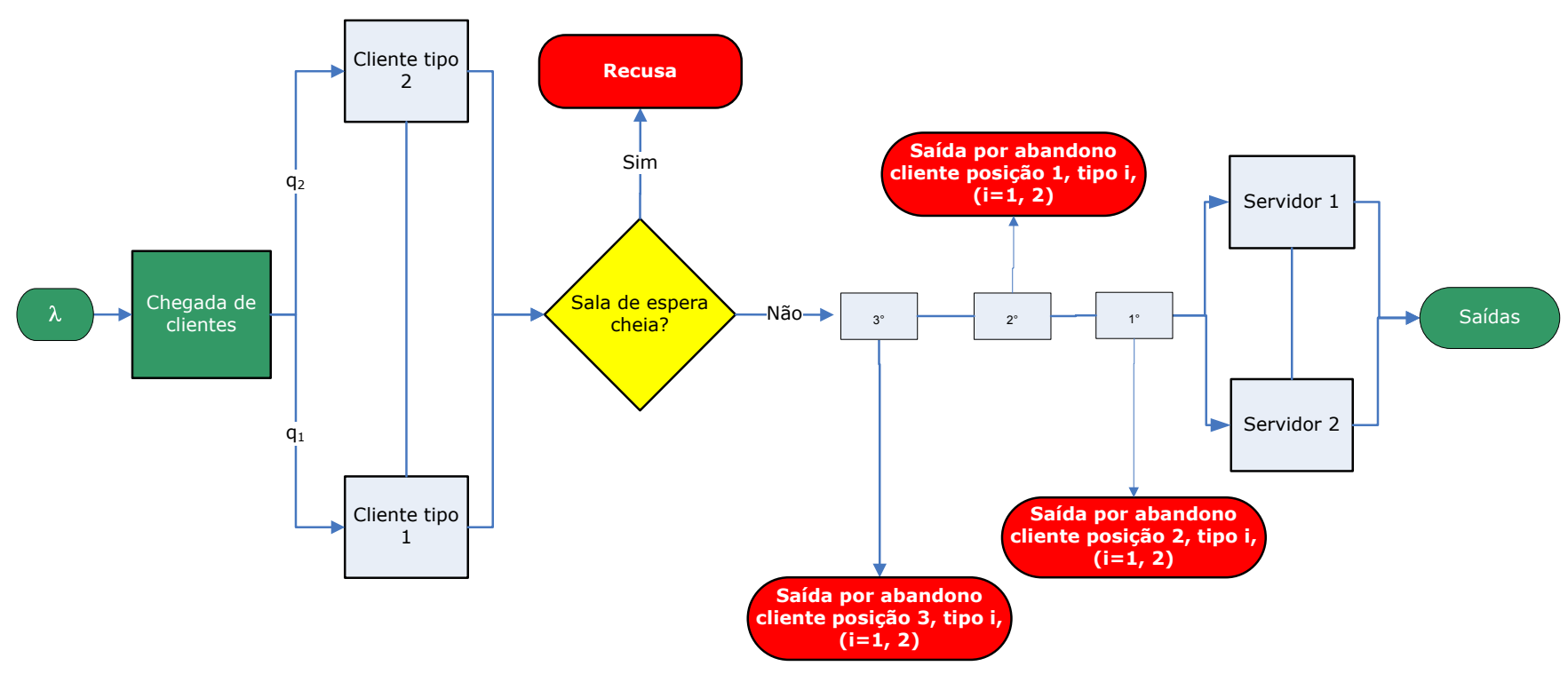

Figura 4.3: Modelo $M / M^{i} / 2 / 3+M^{i}(n)(i=2)$.

Neste caso o sistema possui 48 estados que serão apresentados detalhadamente em Anexo.

Conforme definido no Capítulo 3 apresentaremos alguns exemplos de estados:

- $(1,1 ; 0,0,0)$ : o sistema possui 1 cliente tipo 1 e 1 cliente tipo 2 sendo atendidos.

- $(0,2 ; 1,1,0)$ : o sistema possui dois clientes tipo 2 sendo atendidos e dois clientes tipo 1 aguardando na fila.

- $(2,0 ; 2,2,1)$ : o sistema possui dois clientes tipo 1 sendo atendidos e três clientes, sendo os 
dois primeiros tipo 2 e o último tipo 1, aguardando na fila.

Em função do grande número de estados, este sistema possui um enorme número de transições, tornando inviável a construção do fluxo do sistema. Desta forma apresentamos abaixo alguns exemplos de transição e em anexo o fluxo completo de transições em formato de tabela.

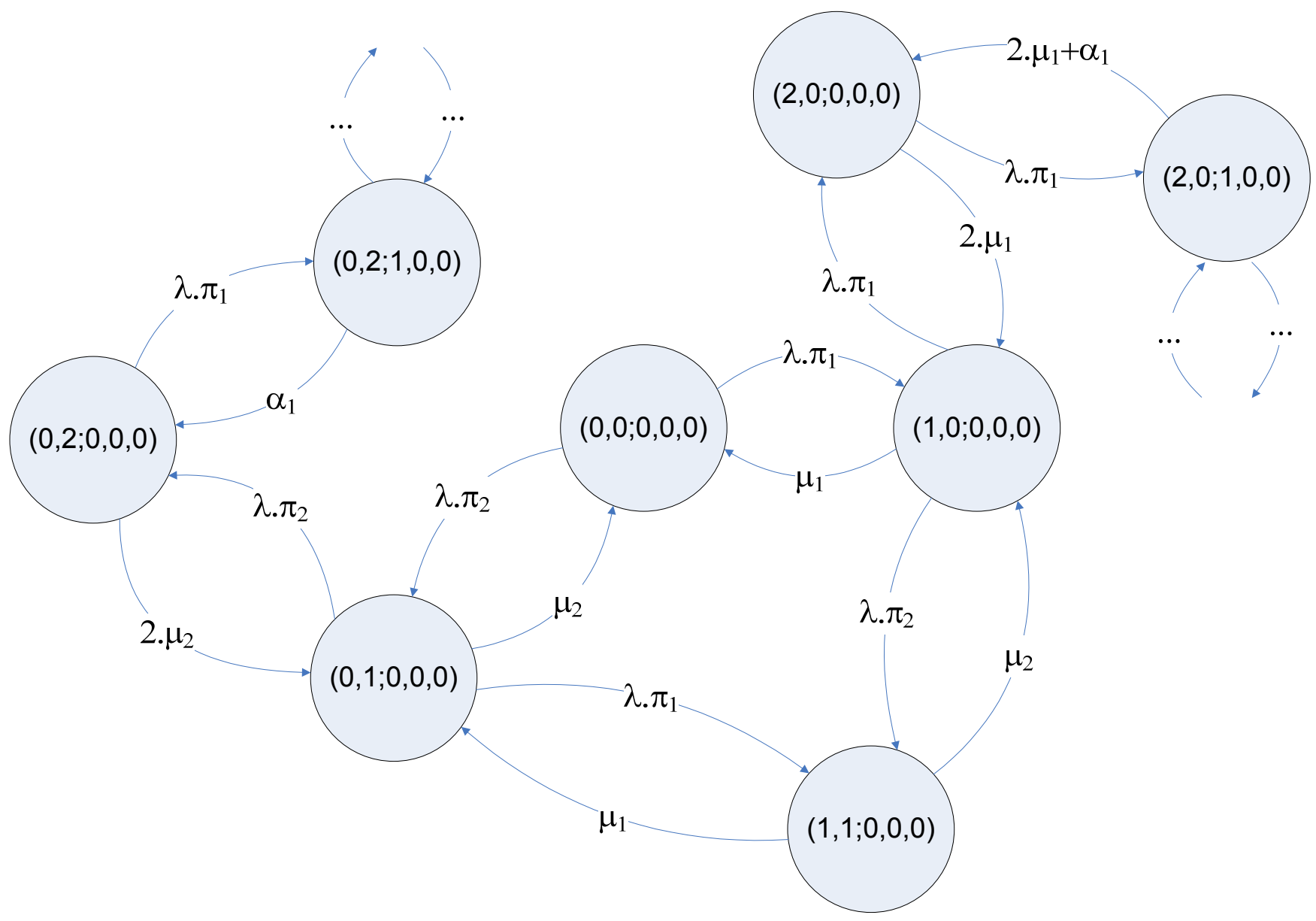

Figura 4.4: Fluxo de alguns estados do modelo $M / M^{i} / 2 / 3+M^{i}(n)(i=2)$.

A partir do fluxo de probabilidade entre os estados, obtemos 48 equações de balanço que estão descritas em anexo, acompanhadas das fórmulas para o cálculo das medidas de desempenho. 
Apresentamos abaixo, na Tabela 4.5, os resultados obtidos para o modelo $M / M^{i} / 2 / 3+M^{i}(n)$, $i=2$, em que clientes tipo 1 possuem distribuição de serviço e paciência Exponencial com taxa 1; e clientes tipo 2 possuem distribuição de serviço e paciência Exponencial com taxa 0,5.

\begin{tabular}{|c|c|c|}
\hline \multicolumn{3}{|c|}{$\begin{array}{l}\text { Aproximação e Simulação } \\
\text { dois tipos de clientes }\end{array}$} \\
\hline \multirow{2}{*}{$\begin{array}{l}\text { Medidas } \\
\text { de } \\
\text { Desenpenho }\end{array}$} & \multicolumn{2}{|c|}{$M / G^{i} / 2 / 3+G^{i}$ - Cenário 1} \\
\hline & Simulação & Aproximação \\
\hline \multirow[t]{2}{*}{$P(W=0)$} & 0,05080 & 0,0576 \\
\hline & $\pm 0,0007$ & - \\
\hline \multirow[t]{2}{*}{$P(A)$} & 0,52880 & 0,4812 \\
\hline & $\pm 0,001$ & - \\
\hline \multirow[t]{2}{*}{$E[Q]$} & 1,75810 & 1,8886 \\
\hline & $\pm 0,0032$ & - \\
\hline \multirow[t]{2}{*}{$\operatorname{Var}(\mathrm{Q})$} & 0,95080 & 1,1008 \\
\hline & $\pm 0,003$ & - \\
\hline \multirow[t]{2}{*}{$E[N]$} & 3,71600 & 3,8470 \\
\hline & $\pm 0,0039$ & - \\
\hline \multirow[t]{2}{*}{$E[W \mid S]$} & 0,73090 & 0,6423 \\
\hline & $\pm 0,003$ & - \\
\hline \multirow[t]{2}{*}{$\operatorname{Var}(\mathrm{W} \mid \mathrm{S})$} & 0,42540 & 0,3092 \\
\hline & $\pm 0,0031$ & - \\
\hline \multirow[t]{2}{*}{$E[W \mid A]$} & 0,58170 & 0,5161 \\
\hline & $\pm 0,002$ & - \\
\hline \multirow[t]{2}{*}{$\operatorname{Var}(\mathrm{W} \mid \mathrm{A})$} & 0,30310 & 0,2239 \\
\hline & $\pm 0,0032$ & - \\
\hline \multirow[t]{2}{*}{$P(W \leq 0.1 \mid S)$} & 0,1602 & 0,1695 \\
\hline & $\pm 0,0015$ & - \\
\hline \multirow[t]{2}{*}{$P(W \leq 0.1 \mid A)$} & 0,1426 & 0,1542 \\
\hline & $\pm 0,0011$ & - \\
\hline \multirow[t]{2}{*}{$P(W \leq 0.2 \mid S)$} & 0,2219 & 0,2395 \\
\hline & $\pm 0,0018$ & - \\
\hline \multirow[t]{2}{*}{$P(W \leq 0.2 \mid A)$} & 0,2699 & 0,2904 \\
\hline & $\pm 0,0013$ & - \\
\hline Parâmetros: & Cenário 1 & \\
\hline$s=2$ & $q_{1}=0,7$ & \\
\hline$r=3$ & Serviços: & \\
\hline Chegadas: Poisson $\lambda=5$ & Tipo 1: Expone & rencial(1) \\
\hline Tipos: $\mathrm{i}=2$ & Tipo 2: Expone & encial $(0,5)$ \\
\hline início: 1.000 & Paciência: & \\
\hline número chegadas: 100.000 & Tipo 1: Expone & encial(1) \\
\hline número réplicas: 30 & Tipo 2: Expone & encial(0,5) \\
\hline
\end{tabular}

Tabela 4.5: Resultados cenário 1, para o modelo $M / M^{i} / 2 / 3+M^{i}(n)(i=2)$. 
Apresentamos abaixo, Tabela 4.6, os resultados obtidos para o modelo $M / M^{i} / 2 / 3+M^{i}(n)$, $i=2$, em que clientes tipo 1 possuem distribuição de serviço e paciência Exponencial com taxa 2; e clientes tipo 2 possuem distribuição de serviço e paciência Erlang com taxa 1.

\begin{tabular}{|c|c|c|}
\hline \multicolumn{3}{|c|}{$\begin{array}{l}\text { Aproximação e Simulação } \\
\text { dois tipos de clientes }\end{array}$} \\
\hline \multirow{2}{*}{$\begin{array}{c}\text { Medidas } \\
\text { de } \\
\text { Desenpenho }\end{array}$} & \multicolumn{2}{|c|}{$M / G^{i} / 2 / 3+G^{i}$ - Cenário 2} \\
\hline & Simulação & Aproximação \\
\hline \multirow[t]{2}{*}{$P(W=0)$} & 0,21300 & 0,2136 \\
\hline & $\pm 0,001$ & - \\
\hline \multirow[t]{2}{*}{$P(A)$} & 0,39600 & 0,3646 \\
\hline & $\pm 0,0009$ & - \\
\hline \multirow[t]{2}{*}{$E[Q]$} & 1,02850 & 1,0506 \\
\hline & $\pm 0,003$ & - \\
\hline \multirow[t]{2}{*}{$\operatorname{Var}(\mathrm{Q})$} & 1,05150 & 1,1433 \\
\hline & $\pm 0,0012$ & - \\
\hline \multirow[t]{2}{*}{$E[N]$} & 2,81870 & 2,8216 \\
\hline & $\pm 0,004$ & - \\
\hline \multirow[t]{2}{*}{$E[W \mid S]$} & 0,22110 & 0,2059 \\
\hline & $\pm 0,0008$ & - \\
\hline \multirow[t]{2}{*}{$\operatorname{Var}(\mathrm{W} \mid \mathrm{S})$} & 0,08460 & 0,0653 \\
\hline & $\pm 0,0004$ & - \\
\hline \multirow[t]{2}{*}{$E[W \mid A]$} & 0,27470 & 0,2446 \\
\hline & $\pm 0,0007$ & - \\
\hline \multirow[t]{2}{*}{$\operatorname{Var}(\mathrm{W} \mid \mathrm{A})$} & 0,07070 & 0,0526 \\
\hline & $\pm 0,0004$ & - \\
\hline \multirow[t]{2}{*}{$P(W \leq 0.1 \mid S)$} & 0,4866 & 0,4790 \\
\hline & $\pm 0,0014$ & - \\
\hline \multirow[t]{2}{*}{$P(W \leq 0.1 \mid A)$} & 0,2923 & 0,3110 \\
\hline & $\pm 0,001$ & - \\
\hline \multirow[t]{2}{*}{$P(W \leq 0.2 \mid S)$} & 0,6086 & 0,6120 \\
\hline & $\pm 0,0013$ & - \\
\hline \multirow[t]{2}{*}{$P(W \leq 0.2 \mid A)$} & 0,5059 & 0,5371 \\
\hline & $\pm 0,0011$ & - \\
\hline Parâmetros: & Cenário 2 & \\
\hline$s=2$ & $q_{1}=0,7$ & \\
\hline$r=3$ & Serviços: & \\
\hline Chegadas: Poisson $\lambda=5$ & Tipo 1: Expone & encial(2) \\
\hline Tipos: $\mathrm{i}=2$ & Tipo 2: Erlangl & \\
\hline início: 1.000 & Paciência: & \\
\hline número chegadas: 100.000 & Tipo 1: Expone & encial(2) \\
\hline número réplicas: 30 & Tipo 2: Erlang( & \\
\hline
\end{tabular}

Tabela 4.6: Resultados cenário 2, para o modelo $M / M^{i} / 2 / 3+M^{i}(n)(i=2)$. 


\section{Capítulo 5}

\section{Conclusões}

\subsection{Considerações Finais}

Neste trabalho apresentamos os resultados desenvolvidos por Whitt em [15], que aproximou o modelo de fila $M / G / s / r+G$ pelo modelo totalmente Markoviano $M / M / s / r+M(n)$. Desenvolvemos dois algoritmos, sendo um para calcular as aproximações propostas por Whitt e outro para fazer a simulação dos modelos de fila. Utilizamos os resultados apresentados por Whitt para validar os algoritmos.

Com base neste trabalho, propusemos uma generalização da aproximação feita por Whitt, para modelos de fila com mais de um tipo de cliente. Onde o modelo de fila $M / G^{i} / s / r+G^{i}$ é aproximado pelo modelo de fila $M / M^{i} / s / r+M^{i}(n)$.

Estudamos o processo estocástico que descreve o modelo $M / M^{i} / s / r+M^{i}(n)$. Através deste estudo, definimos as medidas de desempenho aproximadas para o modelo $M / M^{i} / s / r+M^{i}(n)$.

As medidas de desempenho aproximadas foram calculadas para os casos particulares $M / M^{i} / 1 / 1+$ $M^{i}(n)(i=2)$ e $M / M^{i} / 2 / 3+M^{i}(n)(i=2)$. Desenvolvemos três outros algoritmos, sendo um para simular um modelo de fila com dois tipos de cliente, outro para o cálculo das medidas de desempenho que desenvolvemos para o modelo $M / M^{i} / 1 / 1+M^{i}(n)$, considerando $i=2$ e um terceiro para o cálculo das medidas de desempenho desenvolvidas para o modelo $M / M^{i} / 2 / 3+M^{i}(n)(i=2)$.

Os resultados obtidos através das aproximações propostas no Capítulo 3, e apresentados na Seção 4.2.3, comprovam que a aproximação é bastante satisfatória.

Como sugestão para próximos passos nessa pesquisa, consideramos que seria oportuno desenvolver um método para descrever o espaço de estados e calcular as probabilidades estacionárias de um modelo Markoviano com maior número de servidores e espaços na fila. Daí seria possível aplicar as expressões obtidas no Capítulo 3 para calcular as diversas medidas de desempenho num sistema mais realista. 


\section{Apêndice A}

\section{Anexo}

\section{A.1 Caso $M / M^{i} / 2 / 3+M^{i}(n), i=2$}

\section{A.1.1 Fluxo de estados}

Segue tabela que contém todo o fluxo de entradas e saídas dos estados do caso $M / M^{i} / 2 / 3+$ $M^{i}(n), i=2$. 


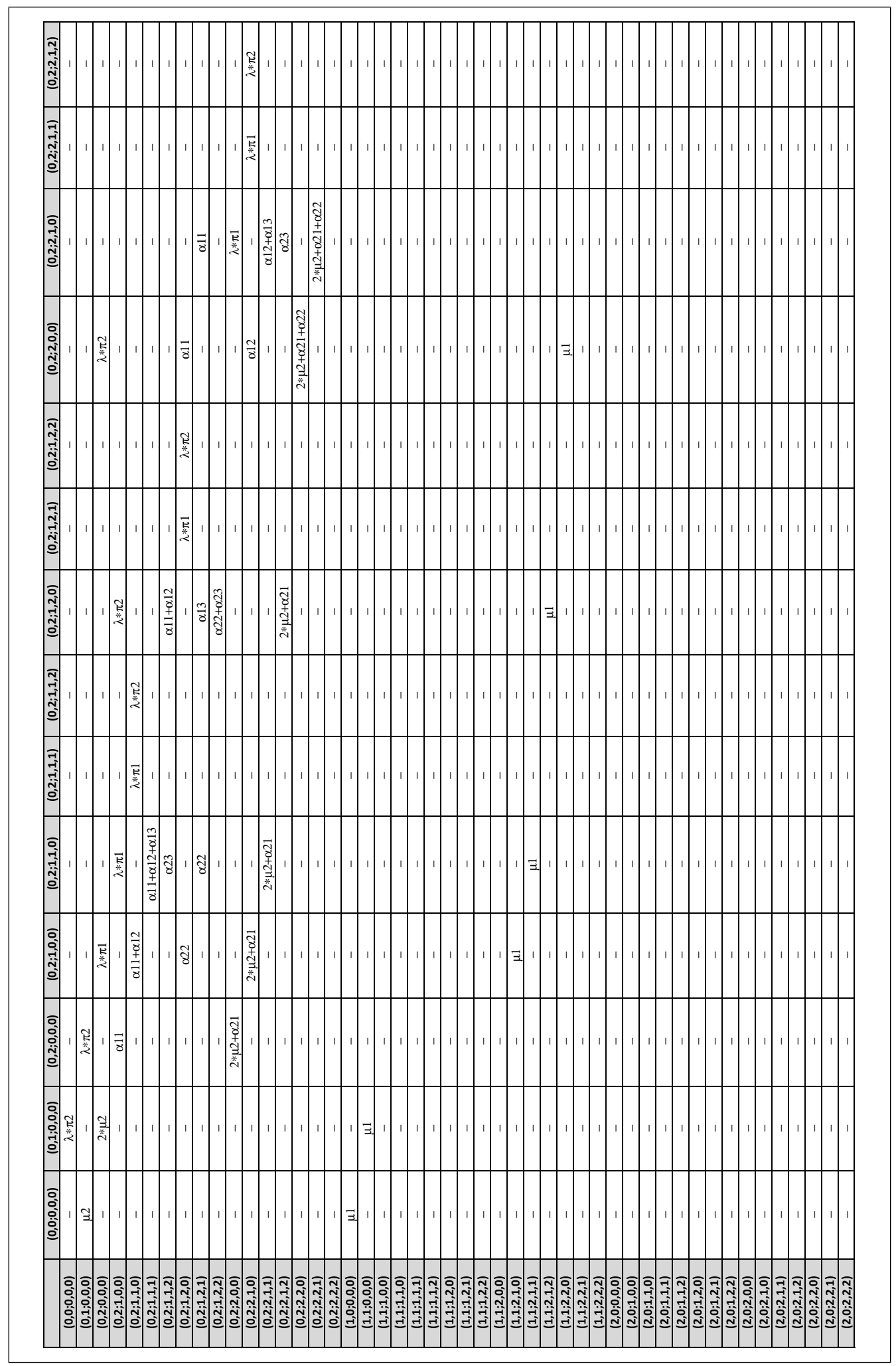

Tabela A.1: Fluxo $M / M^{i} / 2 / 3+M^{i}(n)(i=2)$ (parte 1). 
A.1. $C A S O M / M^{I} / 2 / 3+M^{I}(N), I=2$

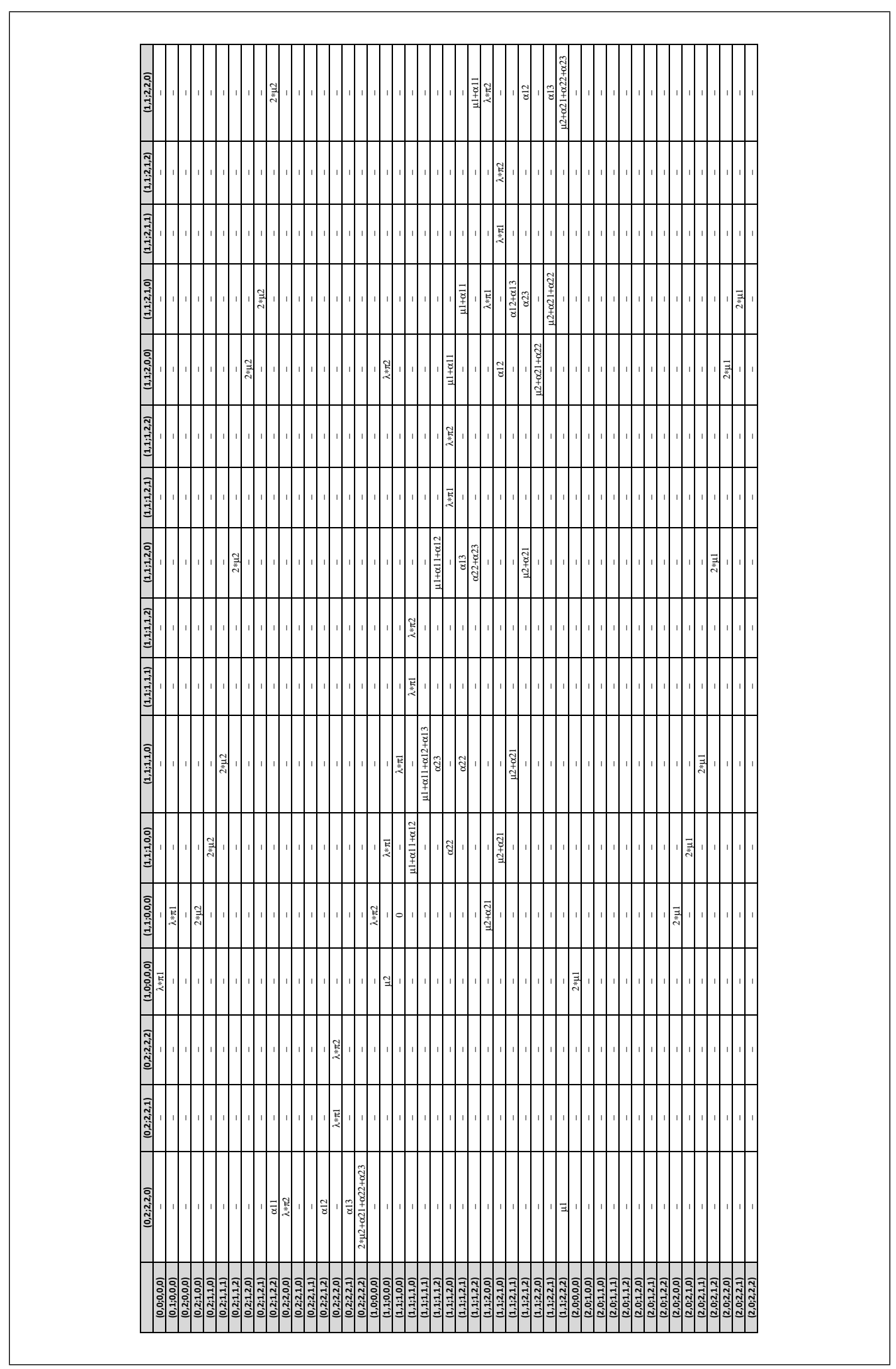

Tabela A.2: Fluxo $M / M^{i} / 2 / 3+M^{i}(n)(i=2)$ (parte 2). 


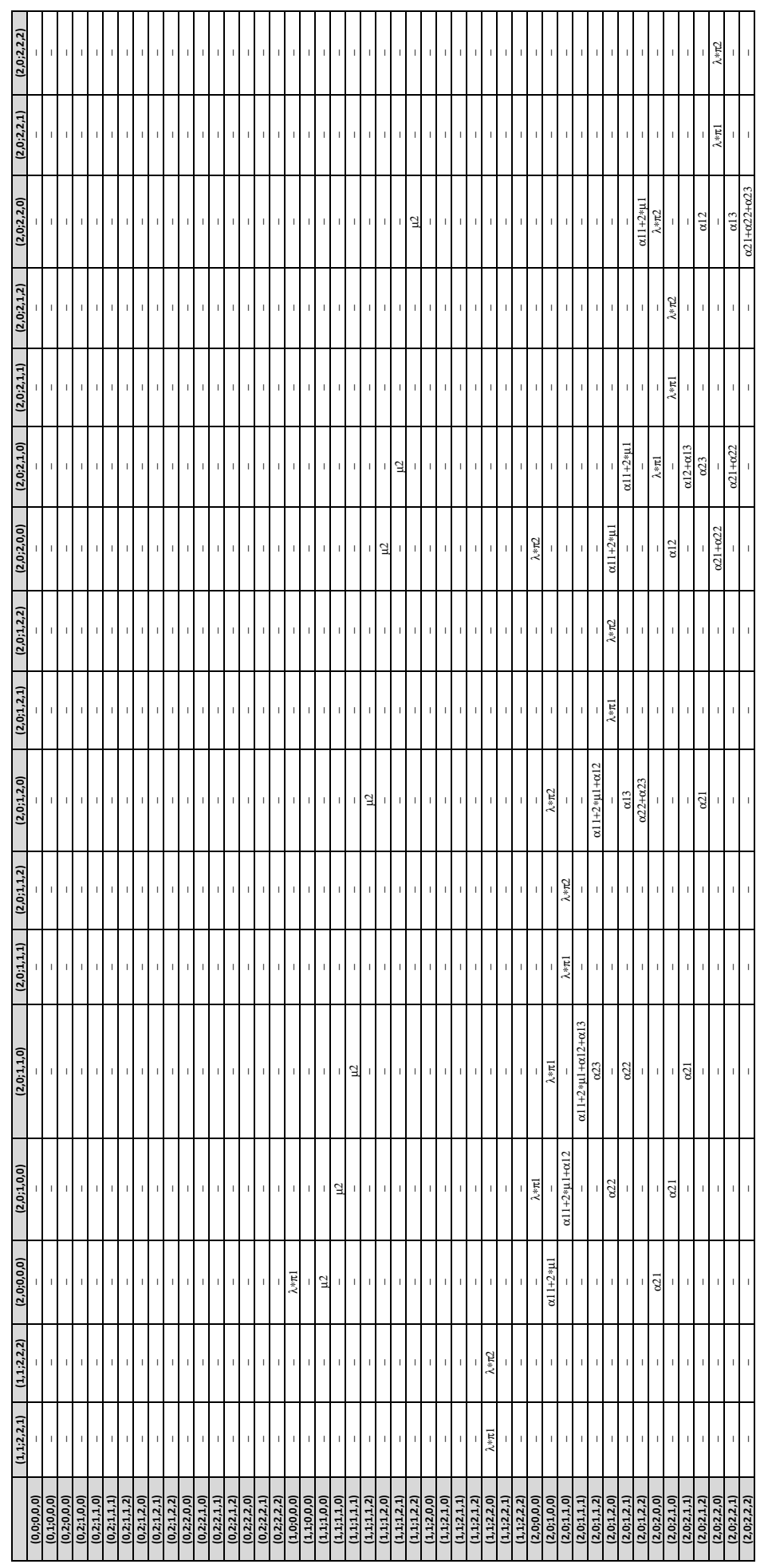

Tabela A.3: Fluxo $M / M^{i} / 2 / 3+M^{i}(n)(i=2)$ (parte 3$)$. 
A.1. CASO $M / M^{I} / 2 / 3+M^{I}(N), I=2$

\section{A.1.2 Equações de balanço}

As equações de balanço são dadas por:

$$
\begin{aligned}
& \pi_{(0,0 ; 0,0,0)} \cdot\left(\lambda_{\cdot 1}+\lambda_{\cdot 2}\right)=\left(\pi_{(1,0 ; 0,0,0)} \cdot \mu_{1}\right)+\left(\pi_{(0,1 ; 0,0,0)} \cdot \mu_{2}\right) \\
& \pi_{(1,0 ; 0,0,0)} \cdot\left(\mu_{1}+\lambda_{\cdot 1}+\lambda_{\cdot 2}\right)=\left(\pi_{(0,0 ; 0,0,0)} \cdot \lambda_{\cdot 1}\right)+\left(\pi_{(1,1 ; 0,0,0)} \cdot \mu_{2}\right)+\left(\pi_{(2,0 ; 0,0,0)} \cdot 2 \cdot \mu_{1}\right) \\
& \pi_{(0,1 ; 0,0,0)} \cdot\left(\mu_{2}+\lambda_{\cdot 1}+\lambda_{\cdot 2}\right)=\left(\pi_{(0,0 ; 0,0,0)} \cdot \lambda_{\cdot 2}\right)+\left(\pi_{(1,1 ; 0,0,0)} \cdot \mu_{1}\right)+\left(\pi_{(0,2 ; 0,0,0)} \cdot 2 \cdot \mu_{2}\right) \\
& \pi_{(1,1 ; 0,0,0)} \cdot\left(\mu_{1}+\mu_{2}+\lambda_{\cdot 1}+\lambda_{\cdot 2}\right)=\left(\pi_{(0,1 ; 0,0,0)} \cdot \lambda_{\cdot 1}\right)+\left(\pi_{(1,0 ; 0,0,0)} \cdot \lambda_{\cdot 2}\right)+ \\
& +\left(\pi_{(1,1 ; 1,0,0)} \cdot \mu_{1}\right)+\left(\pi_{(1,1 ; 1,0,0)} \cdot \alpha_{11}\right)+\left(\pi_{(1,1 ; 2,0,0)} \cdot \mu_{2}\right)+\left(\pi_{(1,1 ; 2,0,0)} \cdot \alpha_{21}\right)+ \\
& +\left(\pi_{(0,2 ; 1,0,0)} \cdot 2 \cdot \mu_{2}\right)+\left(\pi_{(2,0 ; 2,0,0)} \cdot 2 \cdot \mu_{1}\right) \\
& \pi_{(2,0 ; 0,0,0)} \cdot\left(2 \cdot \mu_{1}+\lambda_{\cdot 1}+\lambda_{\cdot 2}\right)=\left(\pi_{(1,0 ; 0,0,0)} \cdot \lambda_{\cdot 1}\right)+\left(\pi_{(1,1 ; 1,0,0)} \cdot \mu_{2}\right)+ \\
& +\left(\pi_{(2,0 ; 1,0,0)} \cdot 2 \cdot \mu_{1}\right)+\left(\pi_{(2,0 ; 2,0,0)} \cdot \alpha_{21}\right)+\left(\pi_{(2,0 ; 1,0,0)} \cdot \alpha_{11}\right) \\
& \pi_{(0,2 ; 0,0,0)} \cdot\left(2 \cdot \mu_{2}+\lambda_{\cdot 1}+\lambda_{\cdot 2}\right)=\left(\pi_{(0,1 ; 0,0,0)} \cdot \lambda_{\cdot 2}\right)+\left(\pi_{(1,1 ; 2,0,0)} \cdot \mu_{1}\right)+ \\
& +\left(\pi_{(0,2 ; 2,0,0)} \cdot 2 \cdot \mu_{2}\right)+\left(\pi_{(0,2 ; 2,0,0)} \cdot \alpha_{21}\right)+\left(\pi_{(0,2 ; 1,0,0)} \cdot \alpha_{11}\right) \\
& \pi_{(1,1 ; 1,0,0)} \cdot\left(\mu_{1}+\mu_{2}+\alpha_{11}+\lambda_{\cdot 1}+\lambda_{\cdot 2}\right)=\left(\pi_{(1,1 ; 0,0,0)} \cdot \lambda_{\cdot 1}\right)+ \\
& +\left(\pi_{(1,1 ; 1,1,0)} \cdot \mu_{1}\right)+\left(\pi_{(1,1 ; 2,1,0)} \cdot \mu_{2}\right)+\left(\pi_{(2,0 ; 2,1,0)} \cdot 2 \cdot \mu_{1}\right)+\left(\pi_{(0,2 ; 1,1,0)} \cdot 2 \cdot \mu_{2}\right)+ \\
& +\left(\pi_{(1,1 ; 1,1,0)} \cdot \alpha_{11}\right)+\left(\pi_{(1,1 ; 1,1,0)} \cdot \alpha_{12}\right)+\left(\pi_{(1,1 ; 2,1,0)} \cdot \alpha_{21}\right)+\left(\pi_{(1,1 ; 1,2,0)} \cdot \alpha_{22}\right) \\
& \pi_{(1,1 ; 2,0,0)} \cdot\left(\mu_{1}+\mu_{2}+\alpha_{21}+\lambda_{\cdot 1}+\lambda_{\cdot 2}\right)=\left(\pi_{(1,1 ; 0,0,0)} \cdot \lambda_{\cdot 2}\right)+ \\
& +\left(\pi_{(1,1 ; 2,2,0)} \cdot \mu_{2}\right)+\left(\pi_{(1,1 ; 1,2,0)} \cdot \mu_{1}\right)+\left(\pi_{(2,0 ; 2,2,0)} \cdot 2 \cdot \mu_{1}\right)+\left(\pi_{(0,2 ; 1,2,0)} \cdot 2 \cdot \mu_{2}\right)+
\end{aligned}
$$




$$
+\left(\pi_{(1,1 ; 2,2,0)} \cdot \alpha_{21}\right)+\left(\pi_{(1,1 ; 2,2,0)} \cdot \alpha_{22}\right)+\left(\pi_{(1,1 ; 1,2,0)} \cdot \alpha_{11}\right)+\left(\pi_{(1,1 ; 2,1,0)} \cdot \alpha_{12}\right)
$$

$$
\begin{aligned}
& \pi_{(2,0 ; 1,0,0)} \cdot\left(2 \cdot \mu_{1}+\alpha_{11}+\lambda_{\cdot 1}+\lambda_{\cdot 2}\right)=\left(\pi_{(2,0 ; 0,0,0)} \cdot \lambda_{\cdot 1}\right)+ \\
&+\left(\pi_{(2,0 ; 1,1,0)} \cdot 2 \cdot \mu_{1}\right)+\left(\pi_{(1,1 ; 1,1,0)} \cdot \mu_{2}\right)+(\left.\pi_{(2,0 ; 1,1,0)} \cdot \alpha_{11}\right)+\left(\pi_{(2,0 ; 1,1,0)} \cdot \alpha_{12}\right)+ \\
&+\left(\pi_{(2,0 ; 2,1,0)} \cdot \alpha_{21}\right)+\left(\pi_{(2,0 ; 1,2,0)} \cdot \alpha_{22}\right)
\end{aligned}
$$

$$
\begin{array}{r}
\pi_{(2,0 ; 2,0,0)} \cdot\left(2 \cdot \mu_{1}+\alpha_{21}+\lambda_{\cdot 1}+\lambda_{\cdot 2}\right)=\left(\pi_{(2,0 ; 0,0,0)} \cdot \lambda_{2}\right)+ \\
+\left(\pi_{(2,0 ; 1,2,0)} \cdot 2 \cdot \mu_{1}\right)+\left(\pi_{(1,1 ; 1,2,0)} \cdot \mu_{2}\right)+\left(\pi_{(2,0 ; 2,2,0)} \cdot \alpha_{21}\right)+\left(\pi_{(2,0 ; 2,2,0)} \cdot \alpha_{22}\right)+ \\
+\left(\pi_{(2,0 ; 1,2,0)} \cdot \alpha_{11}\right)+\left(\pi_{(2,0 ; 2,1,0)} \cdot \alpha_{12}\right)
\end{array}
$$

$$
\begin{array}{r}
\pi_{(0,2 ; 1,0,0)} \cdot\left(2 \cdot \mu_{2}+\alpha_{11}+\lambda_{\cdot 1}+\lambda_{\cdot 2}\right)=\left(\pi_{(0,2 ; 0,0,0)} \cdot \lambda_{\cdot 1}\right)+ \\
+\left(\pi_{(0,2 ; 2,1,0)} \cdot 2 \cdot \mu_{2}\right)+\left(\pi_{(1,1 ; 2,1,0)} \cdot \mu_{1}\right)+\left(\pi_{(0,2 ; 1,1,0)} \cdot \alpha_{11}\right)+\left(\pi_{(0,2 ; 1,1,0)} \cdot \alpha_{12}\right)+ \\
+\left(\pi_{(0,2 ; 2,1,0)} \cdot \alpha_{21}\right)+\left(\pi_{(0,2 ; 1,2,0)} \cdot \alpha_{22}\right)
\end{array}
$$

$$
\begin{aligned}
& \pi_{(0,2 ; 2,0,0)} \cdot\left(2 \cdot \mu_{2}+\alpha_{21}+\lambda_{\cdot 1}+\lambda_{\cdot 2}\right)=\left(\pi_{(0,2 ; 0,0,0)} \cdot \lambda_{\cdot 2}\right)+ \\
&+\left(\pi_{(0,2 ; 2,2,0)} \cdot 2 \cdot \mu_{2}\right)+\left(\pi_{(1,1 ; 2,2,0)} \cdot \mu_{1}\right)+(\left.\pi_{(0,2 ; 2,2,0)} \cdot \alpha_{21}\right)+\left(\pi_{(0,2 ; 2,2,0)} \cdot \alpha_{22}\right)+ \\
&+\left(\pi_{(0,2 ; 1,2,0)} \cdot \alpha_{11}\right)+\left(\pi_{(0,2 ; 2,1,0)} \cdot \alpha_{12}\right)
\end{aligned}
$$

$$
\begin{array}{r}
\pi_{(1,1 ; 1,1,0)} \cdot\left(\mu_{2}+\mu_{1}+\alpha_{11}+\alpha_{12}+\lambda_{\cdot 1}+\lambda_{\cdot 2}\right)=\left(\pi_{(1,1 ; 1,0,0)} \cdot \lambda_{\cdot 1}\right)+ \\
+\left(\pi_{(1,1 ; 1,1,1)} \cdot \mu_{1}\right)+\left(\pi_{(1,1 ; 2,1,1)} \cdot \mu_{2}\right)+\left(\pi_{(2,0 ; 2,1,1)} \cdot 2 \cdot \mu_{1}\right)+\left(\pi_{(0,2 ; 1,1,1)} \cdot 2 \cdot \mu_{2}\right)+ \\
+\left(\pi_{(1,1 ; 1,1,1)} \cdot \alpha_{11}\right)+\left(\pi_{(1,1 ; 1,1,1)} \cdot \alpha_{12}\right)+\left(\pi_{(1,1 ; 1,1,1)} \cdot \alpha_{13}\right)+\left(\pi_{(1,1 ; 2,1,1)} \cdot \alpha_{21}\right)+ \\
+\left(\pi_{(1,1 ; 1,2,1)} \cdot \alpha_{22}\right)+\left(\pi_{(1,1 ; 1,1,2)} \cdot \alpha_{23}\right)
\end{array}
$$

$$
\begin{array}{r}
\pi_{(1,1 ; 1,2,0)} \cdot\left(\mu_{2}+\mu_{1}+\alpha_{11}+\alpha_{22}+\lambda_{\cdot 1}+\lambda_{\cdot 2}\right)=\left(\pi_{(1,1 ; 1,0,0)} \cdot \lambda_{\cdot 2}\right)+ \\
+\left(\pi_{(1,1 ; 1,1,2)} \cdot \mu_{1}\right)+\left(\pi_{(1,1 ; 2,1,2)} \cdot \mu_{2}\right)+\left(\pi_{(2,0 ; 2,1,2)} \cdot 2 \cdot \mu_{1}\right)+\left(\pi_{(0,2 ; 1,1,2)} \cdot 2 \cdot \mu_{2}\right)+ \\
+\left(\pi_{(1,1 ; 1,1,2)} \cdot \alpha_{11}\right)+\left(\pi_{(1,1 ; 1,1,2)} \cdot \alpha_{12}\right)+\left(\pi_{(1,1 ; 1,2,1)} \cdot \alpha_{13}\right)+\left(\pi_{(1,1 ; 2,1,2)} \cdot \alpha_{21}\right)+ \\
+\left(\pi_{(1,1 ; 1,2,2)} \cdot \alpha_{22}\right)+\left(\pi_{(1,1 ; 1,2,2)} \cdot \alpha_{23}\right)
\end{array}
$$




$$
\begin{array}{r}
\pi_{(1,1 ; 2,1,0)} \cdot\left(\mu_{2}+\mu_{1}+\alpha_{21}+\alpha_{12}+\lambda_{\cdot 1}+\lambda_{\cdot 2}\right)=\left(\pi_{(1,1 ; 2,0,0)} \cdot \lambda_{\cdot 1}\right)+ \\
+\left(\pi_{(1,1 ; 1,2,1)} \cdot \mu_{1}\right)+\left(\pi_{(1,1 ; 2,2,1)} \cdot \mu_{2}\right)+\left(\pi_{(2,0 ; 2,2,1)} \cdot 2 \cdot \mu_{1}\right)+\left(\pi_{(0,2 ; 1,2,1)} \cdot 2 \cdot \mu_{2}\right)+ \\
+\left(\pi_{(1,1 ; 1,2,1)} \cdot \alpha_{11}\right)+\left(\pi_{(1,1 ; 2,1,1)} \cdot \alpha_{12}\right)+\left(\pi_{(1,1 ; 2,1,1)} \cdot \alpha_{13}\right)+\left(\pi_{(1,1 ; 2,2,1)} \cdot \alpha_{21}\right)+ \\
+\left(\pi_{(1,1 ; 2,2,1)} \cdot \alpha_{22}\right)+\left(\pi_{(1,1 ; 2,1,2)} \cdot \alpha_{23}\right)
\end{array}
$$

$$
\begin{array}{r}
\pi_{(1,1 ; 2,2,0)} \cdot\left(\mu_{2}+\mu_{1}+\alpha_{21}+\alpha_{22}+\lambda_{\cdot 1}+\lambda_{\cdot 2}\right)=\left(\pi_{(1,1 ; 2,0,0)} \cdot \lambda_{\cdot 2}\right)+ \\
+\left(\pi_{(1,1 ; 1,2,2)} \cdot \mu_{1}\right)+\left(\pi_{(1,1 ; 2,2,2)} \cdot \mu_{2}\right)+\left(\pi_{(2,0 ; 2,2,2)} \cdot 2 \cdot \mu_{1}\right)+\left(\pi_{(0,2 ; 1,2,2)} \cdot 2 \cdot \mu_{2}\right)+ \\
+\left(\pi_{(1,1 ; 1,2,2)} \cdot \alpha_{11}\right)+\left(\pi_{(1,1 ; 2,1,2)} \cdot \alpha_{12}\right)+\left(\pi_{(1,1 ; 2,2,1)} \cdot \alpha_{13}\right)+\left(\pi_{(1,1 ; 2,2,2)} \cdot \alpha_{21}\right)+ \\
+\left(\pi_{(1,1 ; 2,2,2)} \cdot \alpha_{22}\right)+\left(\pi_{(1,1 ; 2,2,2)} \cdot \alpha_{23}\right)
\end{array}
$$

$$
\begin{array}{r}
\pi_{(2,0 ; 1,1,0)} \cdot\left(2 \cdot \mu_{1}+\alpha_{11}+\alpha_{12}+\lambda_{\cdot 1}+\lambda_{\cdot 2}\right)=\left(\pi_{(2,0 ; 1,0,0)} \cdot \lambda_{\cdot 1}\right)+ \\
+\left(\pi_{(2,0 ; 1,1,1)} \cdot 2 \cdot \mu_{1}\right)+\left(\pi_{(1,1 ; 1,1,1)} \cdot \mu_{2}\right)+\left(\pi_{(2,0 ; 1,1,1)} \cdot \alpha_{11}\right)+\left(\pi_{(2,0 ; 1,1,1)} \cdot \alpha_{12}\right)+ \\
+\left(\pi_{(2,0 ; 1,1,1)} \cdot \alpha_{13}\right)+\left(\pi_{(2,0 ; 2,1,1)} \cdot \alpha_{21}\right)+\left(\pi_{(2,0 ; 1,2,1)} \cdot \alpha_{22}\right)+\left(\pi_{(2,0 ; 1,1,2)} \cdot \alpha_{23}\right)
\end{array}
$$

$$
\begin{array}{r}
\pi_{(2,0 ; 1,2,0)} \cdot\left(2 \cdot \mu_{1}+\alpha_{11}+\alpha_{22}+\lambda_{\cdot 1}+\lambda_{\cdot 2}\right)=\left(\pi_{(2,0 ; 1,0,0)} \cdot \lambda_{\cdot 2}\right)+ \\
+\left(\pi_{(2,0 ; 1,1,2)} \cdot 2 \cdot \mu_{1}\right)+\left(\pi_{(1,1 ; 1,1,2)} \cdot \mu_{2}\right)+\left(\pi_{(2,0 ; 1,1,2)} \cdot \alpha_{11}\right)+\left(\pi_{(2,0 ; 1,1,2)} \cdot \alpha_{12}\right)+ \\
+\left(\pi_{(2,0 ; 1,2,1)} \cdot \alpha_{13}\right)+\left(\pi_{(2,0 ; 2,1,2)} \cdot \alpha_{21}\right)+\left(\pi_{(2,0 ; 1,2,2)} \cdot \alpha_{22}\right)+\left(\pi_{(2,0 ; 1,2,2)} \cdot \alpha_{23}\right)
\end{array}
$$

$$
\begin{array}{r}
\pi_{(2,0 ; 2,1,0)} \cdot\left(2 \cdot \mu_{1}+\alpha_{21}+\alpha_{12}+\lambda_{\cdot 1}+\lambda_{\cdot 2}\right)=\left(\pi_{(2,0 ; 2,0,0)} \cdot \lambda_{\cdot 1}\right)+ \\
+\left(\pi_{(2,0 ; 1,2,1)} \cdot 2 \cdot \mu_{1}\right)+\left(\pi_{(1,1 ; 1,2,1)} \cdot \mu_{2}\right)+\left(\pi_{(2,0 ; 1,2,1)} \cdot \alpha_{11}\right)+\left(\pi_{(2,0 ; 2,1,1)} \cdot \alpha_{12}\right)+ \\
+\left(\pi_{(2,0 ; 2,1,1)} \cdot \alpha_{13}\right)+\left(\pi_{(2,0 ; 2,2,1)} \cdot \alpha_{21}\right)+\left(\pi_{(2,0 ; 2,2,1)} \cdot \alpha_{22}\right)+\left(\pi_{(2,0 ; 2,1,2)} \cdot \alpha_{23}\right)
\end{array}
$$

$$
\begin{array}{r}
\pi_{(2,0 ; 2,2,0)} \cdot\left(2 \cdot \mu_{1}+\alpha_{21}+\alpha_{22}+\lambda_{\cdot 1}+\lambda_{\cdot 2}\right)=\left(\pi_{(2,0 ; 2,0,0)} \cdot \lambda_{\cdot 2}\right)+ \\
+\left(\pi_{(2,0 ; 1,2,2)} \cdot 2 \cdot \mu_{1}\right)+\left(\pi_{(1,1 ; 1,2,2)} \cdot \mu_{2}\right)+\left(\pi_{(2,0 ; 1,2,2)} \cdot \alpha_{11}\right)+\left(\pi_{(2,0 ; 2,1,2)} \cdot \alpha_{12}\right)+ \\
+\left(\pi_{(2,0 ; 2,2,1)} \cdot \alpha_{13}\right)+\left(\pi_{(2,0 ; 2,2,2)} \cdot \alpha_{21}\right)+\left(\pi_{(2,0 ; 2,2,2)} \cdot \alpha_{22}\right)+\left(\pi_{(2,0 ; 2,2,2)} \cdot \alpha_{23}\right)
\end{array}
$$




$$
\begin{array}{r}
\pi_{(0,2 ; 1,1,0)} \cdot\left(2 \cdot \mu_{2}+\alpha_{11}+\alpha_{12}+\lambda_{\cdot 1}+\lambda_{\cdot 2}\right)=\left(\pi_{(0,2 ; 1,0,0)} \cdot \lambda_{\cdot 1}\right)+ \\
+\left(\pi_{(0,2 ; 2,1,1)} \cdot 2 \cdot \mu_{2}\right)+\left(\pi_{(1,1 ; 2,1,1)} \cdot \mu_{1}\right)+\left(\pi_{(0,2 ; 1,1,1)} \cdot \alpha_{11}\right)+\left(\pi_{(0,2 ; 1,1,1)} \cdot \alpha_{12}\right)+ \\
+\left(\pi_{(0,2 ; 1,1,1)} \cdot \alpha_{13}\right)+\left(\pi_{(0,2 ; 2,1,1)} \cdot \alpha_{21}\right)+\left(\pi_{(0,2 ; 1,2,1)} \cdot \alpha_{22}\right)+\left(\pi_{(0,2 ; 1,1,2)} \cdot \alpha_{23}\right)
\end{array}
$$

$$
\begin{array}{r}
\pi_{(0,2 ; 1,2,0)} \cdot\left(2 \cdot \mu_{2}+\alpha_{11}+\alpha_{22}+\lambda_{\cdot 1}+\lambda_{\cdot 2}\right)=\left(\pi_{(0,2 ; 1,0,0)} \cdot \lambda_{\cdot 2}\right)+ \\
+\left(\pi_{(0,2 ; 2,1,2)} \cdot 2 \cdot \mu_{2}\right)+\left(\pi_{(1,1 ; 2,1,2)} \cdot \mu_{1}\right)+\left(\pi_{(0,2 ; 1,1,2)} \cdot \alpha_{11}\right)+\left(\pi_{(0,2 ; 1,1,2)} \cdot \alpha_{12}\right)+ \\
+\left(\pi_{(0,2 ; 1,2,1)} \cdot \alpha_{13}\right)+\left(\pi_{(0,2 ; 2,1,2)} \cdot \alpha_{21}\right)+\left(\pi_{(0,2 ; 1,2,2)} \cdot \alpha_{22}\right)+\left(\pi_{(0,2 ; 1,2,2)} \cdot \alpha_{23}\right)
\end{array}
$$

$$
\begin{array}{r}
\pi_{(0,2 ; 2,1,0)} \cdot\left(2 \cdot \mu_{2}+\alpha_{21}+\alpha_{12}+\lambda_{\cdot 1}+\lambda_{\cdot 2}\right)=\left(\pi_{(0,2 ; 2,0,0)} \cdot \lambda_{\cdot 1}\right)+ \\
+\left(\pi_{(1,1 ; 2,2,1)} \cdot \mu_{1}\right)+\left(\pi_{(0,2 ; 2,2,1)} \cdot 2 \cdot \mu_{2}\right)+\left(\pi_{(0,2 ; 1,2,1)} \cdot \alpha_{11}\right)+\left(\pi_{(0,2 ; 2,1,1)} \cdot \alpha_{12}\right)+ \\
+\left(\pi_{(0,2 ; 2,1,1)} \cdot \alpha_{13}\right)+\left(\pi_{(0,2 ; 2,2,1)} \cdot \alpha_{21}\right)+\left(\pi_{(0,2 ; 2,2,1)} \cdot \alpha_{22}\right)+\left(\pi_{(0,2 ; 2,1,2)} \cdot \alpha_{23}\right)
\end{array}
$$

$$
\begin{array}{r}
\pi_{(0,2 ; 2,2,0)} \cdot\left(2 \cdot \mu_{2}+\alpha_{21}+\alpha_{22}+\lambda_{\cdot 1}+\lambda_{\cdot 2}\right)=\left(\pi_{(0,2 ; 2,0,0)} \cdot \lambda_{\cdot 2}\right)+ \\
+\left(\pi_{(0,2 ; 2,2,2)} \cdot 2 \cdot \mu_{2}\right)+\left(\pi_{(1,1 ; 2,2,2)} \cdot \mu_{1}\right)+\left(\pi_{(0,2 ; 1,2,2)} \cdot \alpha_{11}\right)+\left(\pi_{(0,2 ; 2,1,2)} \cdot \alpha_{12}\right)+ \\
+\left(\pi_{(0,2 ; 2,2,1)} \cdot \alpha_{13}\right)+\left(\pi_{(0,2 ; 2,2,2)} \cdot \alpha_{21}\right)+\left(\pi_{(0,2 ; 2,2,2)} \cdot \alpha_{22}\right)+\left(\pi_{(0,2 ; 2,2,2)} \cdot \alpha_{23}\right)
\end{array}
$$$$
\pi_{(1,1 ; 1,1,1)} \cdot\left(\mu_{1}+\mu_{2}+\alpha_{11}+\alpha_{12}+\alpha_{13}\right)=\left(\pi_{(1,1 ; 1,1,0)} \cdot \lambda_{\cdot 1}\right)
$$$$
\pi_{(1,1 ; 1,1,2)} \cdot\left(\mu_{1}+\mu_{2}+\alpha_{11}+\alpha_{12}+\alpha_{23}\right)=\left(\pi_{(1,1 ; 1,1,0)} \cdot \lambda_{\cdot 2}\right)
$$$$
\pi_{(1,1 ; 1,2,1)} \cdot\left(\mu_{1}+\mu_{2}+\alpha_{11}+\alpha_{22}+\alpha_{13}\right)=\left(\pi_{(1,1 ; 1,2,0)} \cdot \lambda_{\cdot 1}\right)
$$$$
\pi_{(1,1 ; 1,2,2)} \cdot\left(\mu_{1}+\mu_{2}+\alpha_{11}+\alpha_{22}+\alpha_{23}\right)=\left(\pi_{(1,1 ; 1,2,0)} \cdot \lambda_{\cdot 2}\right)
$$ 


$$
\begin{aligned}
& \pi_{(1,1 ; 2,1,1)} \cdot\left(\mu_{1}+\mu_{2}+\alpha_{21}+\alpha_{12}+\alpha_{13}\right)=\left(\pi_{(1,1 ; 2,1,0)} \cdot \lambda \cdot 1\right) \\
& \pi_{(1,1 ; 2,1,2)} \cdot\left(\mu_{1}+\mu_{2}+\alpha_{21}+\alpha_{12}+\alpha_{23}\right)=\left(\pi_{(1,1 ; 2,1,0)} \cdot \lambda \cdot 2\right) \\
& \pi_{(1,1 ; 2,2,1)} \cdot\left(\mu_{1}+\mu_{2}+\alpha_{21}+\alpha_{22}+\alpha_{13}\right)=\left(\pi_{(1,1 ; 2,2,0)} \cdot \lambda \cdot 1\right) \\
& \pi_{(1,1 ; 2,2,2)} \cdot\left(\mu_{1}+\mu_{2}+\alpha_{21}+\alpha_{22}+\alpha_{23}\right)=\left(\pi_{(1,1 ; 2,2,0)} \cdot \lambda \cdot 2\right) \\
& \pi_{(2,0 ; 2,1,2)} \cdot\left(2 . \mu_{1}+\alpha_{21}+\alpha_{12}+\alpha_{23}\right)=\left(\pi_{(2,0 ; 2,1,0)} \cdot \lambda \cdot 2\right) \\
& \pi_{(2,0 ; 1,1,1)} \cdot\left(2 . \mu_{1}+\alpha_{11}+\alpha_{12}+\alpha_{13}\right)=\left(\pi_{(2,0 ; 1,1,0)} \cdot \lambda \cdot 1\right) \\
& \pi_{(2,0 ; 1,2,1)} \cdot\left(2 . \mu_{1}+\alpha_{11}+\alpha_{22}+\alpha_{13}\right)=\left(\pi_{(2,0 ; 1,2,0)} \cdot \lambda \cdot 1\right) \\
& \pi_{(2,0 ; 1,1,2)} \cdot\left(2 . \mu_{1}+\alpha_{11}+\alpha_{12}+\alpha_{23}\right)=\left(\pi_{(2,0 ; 1,1,0)} \cdot \lambda \cdot 2\right)
\end{aligned}
$$




$$
\begin{aligned}
& \pi_{(2,0 ; 2,2,2)} \cdot\left(2 \cdot \mu_{1}+\alpha_{21}+\alpha_{22}+\alpha_{23}\right)=\left(\pi_{(2,0 ; 2,2,0)} \cdot \lambda_{\cdot 2}\right) \\
& \pi_{(0,2 ; 1,1,1)} \cdot\left(2 . \mu_{2}+\alpha_{11}+\alpha_{12}+\alpha_{13}\right)=\left(\pi_{(0,2 ; 1,1,0)} \cdot \lambda_{\cdot 1}\right) \\
& \pi_{(0,2 ; 1,1,2)} \cdot\left(2 . \mu_{2}+\alpha_{11}+\alpha_{12}+\alpha_{23}\right)=\left(\pi_{(0,2 ; 1,1,0)} \cdot \lambda \cdot 2\right) \\
& \pi_{(0,2 ; 1,2,1)} \cdot\left(2 . \mu_{2}+\alpha_{11}+\alpha_{22}+\alpha_{13}\right)=\left(\pi_{(0,2 ; 1,2,0)} \cdot \lambda \cdot 1\right) \\
& \pi_{(0,2 ; 1,2,2)} \cdot\left(2 . \mu_{2}+\alpha_{11}+\alpha_{22}+\alpha_{23}\right)=\left(\pi_{(0,2 ; 1,2,0)} \cdot \lambda \cdot 2\right) \\
& \pi_{(0,2 ; 2,2,1)} \cdot\left(2 . \mu_{2}+\alpha_{21}+\alpha_{22}+\alpha_{13}\right)=\left(\pi_{(0,2 ; 2,2,0)} \cdot \lambda \cdot 1\right) \\
& \pi_{(0,2 ; 2,1,2)} \cdot\left(2 . \mu_{2}+\alpha_{21}+\alpha_{12}+\alpha_{23}\right)=\left(\pi_{(0,2 ; 2,1,0)} \cdot \lambda \cdot 2\right) \\
& \pi_{(0,2 ; 2,1,1)} \cdot\left(2 . \mu_{2}+\alpha_{21}+\alpha_{12}+\alpha_{13}\right)=\left(\pi_{(0,2 ; 2,1,0)} \cdot \lambda \cdot \mu_{1}\right)
\end{aligned}
$$

\section{A.1.3 Medidas de desempenho}

A probabilidade de recusa é definida pelos estados em que a entrada de um cliente não é possível, ou seja, existem dois clientes sendo servidos e três clientes aguardando na fila.

$$
P(\text { recusa })=\pi_{(0,2 ; 1,1,1)}+\pi_{(0,2 ; 1,1,2)}+\pi_{(0,2 ; 1,2,1)}+\pi_{(0,2 ; 1,2,2)}+\pi_{(0,2 ; 2,1,1)}+\pi_{(0,2 ; 2,1,2)}+
$$




$$
\begin{aligned}
& +\pi_{(0,2 ; 2,2,1)}+\pi_{(0,2 ; 2,2,2)}+\pi_{(1,1 ; 1,1,1)}+\pi_{(1,1 ; 1,1,2)}+\pi_{(1,1 ; 1,2,1)}+\pi_{(1,1 ; 1,2,2)}+ \\
& +\pi_{(1,1 ; 2,1,1)}+\pi_{(1,1 ; 2,1,2)}+\pi_{(1,1 ; 2,2,1)}+\pi_{(1,1 ; 2,2,2)}+\pi_{(2,0 ; 1,1,1)}+\pi_{(2,0 ; 1,1,2)}+ \\
& \quad+\pi_{(2,0 ; 1,2,1)}+\pi_{(2,0 ; 1,2,2)}+\pi_{(2,0 ; 2,1,1)}+\pi_{(2,0 ; 2,1,2)}+\pi_{(2,0 ; 2,2,1)}+\pi_{(2,0 ; 2,2,2)}
\end{aligned}
$$

A probabilidade estacionária de um cliente entrar no sistema, definida em 3.30, é dada por:

$$
\begin{array}{r}
\pi^{a}[01]=\pi_{(0,0 ; 0,0,0)} /(1-P(\text { recusa })) \\
\pi^{a}[02]=\left(\pi_{(0,1 ; 0,0,0)}+\pi_{(1,0 ; 0,0,0)}\right) /(1-P(\text { recusa })) \\
\pi^{a}[03]=\left(\pi_{(1,1 ; 0,0,0)}+\pi_{(2,0 ; 0,0,0)}+\pi_{(0,2 ; 0,0,0)}\right) /(1-P(\text { recusa })) \\
\pi^{a}[04]=\left(\pi_{(0,2 ; 2,0,0)}+\pi_{(1,1 ; 2,0,0)}+\pi_{(2,0 ; 1,0,0)}+\pi_{(2,0 ; 2,0,0)}+\pi_{(1,1 ; 1,0,0)}+\right. \\
\left.+\pi_{(0,2 ; 1,0,0)}\right) /(1-P(\text { recusa })) ; \\
\pi^{a}[05]=\left(\pi_{(2,0 ; 2,1,0)}+\pi_{(2,0 ; 1,1,0)}+\pi_{(1,1 ; 2,2,0)}+\pi_{(1,1 ; 2,1,0)}+\pi_{(2,0 ; 1,2,0)}+\right. \\
\left.+\pi_{(2,0 ; 2,2,0)}+\pi_{(1,1 ; 1,2,0)}+\pi_{(1,1 ; 1,1,0)}+\pi_{(0,2 ; 2,2,0)}+\pi_{(0,2 ; 2,1,0)}+\pi_{(0,2 ; 1,2,0)}+\pi_{(0,2 ; 1,1,0)}\right) /(1-P(\text { recusa })) \\
\pi^{a}[06]=\left(\pi_{(1,1 ; 1,2,2)}+\pi_{(1,1 ; 1,1,2)}+\pi_{(1,1 ; 1,1,1)}+\pi_{(0,2 ; 2,2,2)}+\pi_{(1,1 ; 1,2,1)}+\right. \\
+\pi_{(0,2 ; 1,1,1)}+\pi_{(0,2 ; 2,2,1)}+\pi_{(0,2 ; 2,1,2)}+\pi_{(0,2 ; 2,1,1)}+\pi_{(0,2 ; 1,2,2)}+\pi_{(0,2 ; 1,2,1)}+ \\
+\pi_{(0,2 ; 1,1,2)}+\pi_{(1,1 ; 2,1,1)}+\pi_{(1,1 ; 2,1,2)}+\pi_{(1,1 ; 2,2,1)}+\pi_{(1,1 ; 2,2,2)}+\pi_{(2,0 ; 1,1,1)}+\pi_{(2,0 ; 1,1,2)}+ \\
\left.+\pi_{(2,0 ; 1,2,1)}+\pi_{(2,0 ; 1,2,2)}+\pi_{(2,0 ; 2,1,1)}+\pi_{(2,0 ; 2,1,2)}+\pi_{(2,0 ; 2,2,1)}+\pi_{(2,0 ; 2,2,2)}\right) /(1-P(\text { recusa })) .
\end{array}
$$

A seguir temos algumas variáveis auxiliares utilizadas nos cálculos.

$$
\begin{array}{r}
A=2 .\left(\mu_{1} . q_{1}+\mu_{2} . q_{2}\right) \\
B=q_{1} . \alpha_{11}+q_{2} . \alpha_{21} \\
C=q_{1} . \alpha_{12}+q_{2} . \alpha_{22} .
\end{array}
$$

$$
\begin{array}{r}
m l 1=\left(\left(1 /\left(A+B+C+\alpha_{13}\right)\right) * q_{1}+\left(1 /\left(A+B+C+\alpha_{23}\right)\right) \cdot q_{2}\right) ; \\
m l 2=\left(\left(1 /\left(A+B+\alpha_{12}\right)\right) * q_{1}+\left(1 /\left(A+B+\alpha_{22}\right)\right) \cdot q_{2}\right) ; \\
m l 3=\left(\left(1 /\left(A+\alpha_{11}\right)\right) * q_{1}+\left(1 /\left(A+\alpha_{21}\right)\right) \cdot q_{2}\right) .
\end{array}
$$

$$
\begin{array}{r}
a 13=\alpha_{13} /\left(A+B+C+\alpha_{13}\right) ; \\
a 23=\alpha_{23} /\left(A+B+C+\alpha_{23}\right) ; \\
a 12=\alpha_{12} /\left(A+B+\alpha_{12}\right) ;
\end{array}
$$




$$
\begin{array}{r}
a 22=\alpha_{22} /\left(A+B+\alpha_{22}\right) ; \\
a 11=\alpha_{11} /\left(A+\alpha_{11}\right) ; \\
a 21=\alpha_{21} /\left(A+\alpha_{21}\right) .
\end{array}
$$

$$
\begin{array}{r}
m 13=1 /\left(A+B+C+\alpha_{13}\right) ; \\
m 23=1 /\left(A+B+C+\alpha_{23}\right) ; \\
m 12=1 /\left(A+B+\alpha_{12}\right) ; \\
m 22=1 /\left(A+B+\alpha_{22}\right) ; \\
m 11=1 /\left(A+\alpha_{11}\right) ; \\
m 21=1 /\left(A+\alpha_{21}\right) .
\end{array}
$$

$$
\begin{array}{r}
g 11=(1-a 13) \cdot(1-a 12) \cdot(1-a 11) ; \\
g 21=(1-a 12) \cdot(1-a 11) ; \\
g 31=(1-a 11) ; \\
g 12=(1-a 23) \cdot(1-a 22) \cdot(1-a 21) ; \\
g 22=(1-a 22) .(1-a 21) ; g 32=(1-a 21) .
\end{array}
$$

As medidas de desempenho são calculadas conforme apresentadas abaixo.

$$
\begin{gathered}
P(S)=p i^{a}[01]+p i^{a}[02]+\left(p i^{a}[03] \cdot\left(\left(q_{1} \cdot\left(1-\left(\alpha_{11} /\left(A+\alpha_{11}\right)\right)\right)\right)+\left(q_{2} \cdot\left(1-\left(\alpha_{21} /\left(A+\alpha_{21}\right)\right)\right)\right)\right)+\right. \\
\left(p i^{a}[04] .\left(\left(q_{1} \cdot\left(\left(1-\left(\alpha_{12} /\left(A+B+\alpha_{12}\right)\right)\right) \cdot\left(1-\left(\alpha_{11} /\left(A+\alpha_{11}\right)\right)\right)\right)\right)+\left(q _ { 2 } \cdot \left(\left(1-\left(a l p h a 22 /\left(A+B+\alpha_{22}\right)\right)\right) .\right.\right.\right.\right. \\
\left.\left.\left..\left(1-\left(\alpha_{21} /\left(A+\alpha_{21}\right)\right)\right)\right)\right)\right)+\left(p i ^ { a } [ 0 5 ] \cdot \left(\left(q _ { 1 } \cdot \left(\left(1-\left(\alpha_{13} /\left(A+B+C+\alpha_{13}\right)\right)\right) \cdot\left(1-\left(\alpha_{12} /\left(A+B+\alpha_{12}\right)\right)\right) .\right.\right.\right.\right. \\
\left.\left..\left(1-\left(\alpha_{11} /\left(A+\alpha_{11}\right)\right)\right)\right)\right)+\left(q _ { 2 } \cdot \left(\left(1-\left(\alpha_{23} /\left(A+B+C+\alpha_{23}\right)\right)\right) \cdot\left(1-\left(\alpha_{22} /\left(A+B+\alpha_{22}\right)\right)\right) .\right.\right. \\
\left.\left.\left.\left..\left(1-\left(\alpha_{21} /\left(A+\alpha_{21}\right)\right)\right)\right)\right)\right)\right) \\
E[W ; S]=\left(p i^{a}[05] .\left(g 11 \cdot q_{1}+g 12 \cdot q_{2}\right) \cdot(m l 1+m l 2+m l 3)\right)+ \\
\left(p i^{a}[04] \cdot\left(g 21 \cdot q_{1}+g 22 \cdot q_{2}\right) \cdot(m l 2+m l 3)\right)+ \\
\left(p i^{a}[03] \cdot\left(g 31 . q_{1}+g 32 . q_{2}\right) .(m l 3)\right)
\end{gathered}
$$




$$
\begin{array}{r}
E\left[W^{2} ; S\right]=\left(p i^{a}[05] .\left(g 11 . q_{1}+g 12 . q_{2}\right) \cdot\left(m l 1^{2}+m l 2^{2}+m l 3^{2}+(m l 1+m l 2+m l 3)^{2}\right)\right)+ \\
\left(p i^{a}[04] \cdot\left(g 21 . q_{1}+g 22 . q_{2}\right) \cdot\left(m l 2^{2}+m l 3^{2}+(m l 2+m l 3)^{2}\right)\right)+ \\
\left(p i^{a}[03] \cdot\left(g 31 . q_{1}+g 32 . q_{2}\right) \cdot 2 .(m l 3)^{2}\right)
\end{array}
$$

$$
\begin{aligned}
& E 1 A=(a 13 \cdot m 13)+((1-a 13) \cdot a 12 \cdot(m 13+m 12))+((1-a 13) \cdot(1-a 12) \cdot a 11 \cdot(m 13+m 12+m 11)) ; \\
& E 2 A=(a 23 \cdot m 23)+((1-a 23) \cdot a 22 \cdot(m 23+m 22))+((1-a 23) \cdot(1-a 22) \cdot a 21 \cdot(m 23+m 22+m 21)) \text {; } \\
& E 3 A=(a 12 \cdot m 12)+((1-a 12) \cdot a 11 \cdot(m 12+m 11)) ; \\
& E 4 A=(a 22 . m 22)+((1-a 22) \cdot a 21 \cdot(m 22+m 21)) ; \\
& E 5 A=(a 11 . m 11) \\
& E 6 A=(a 21 . m 21) \text {. }
\end{aligned}
$$

$E\left[W_{N} 1_{A}\right]=p i^{a}[05] \cdot\left(q_{1} \cdot E 1 A+q_{2} \cdot E 2 A\right)+p i^{a}[04] \cdot\left(q_{1} \cdot E 3 A+q_{2} \cdot E 4 A\right)+p i^{a}[03] \cdot\left(q_{1} \cdot E 5 A+q_{2} \cdot E 6 A\right)$

$$
\begin{aligned}
& E 1 A 2=\left(a 13 \cdot 2 \cdot m 13^{2}\right)+\left((1-a 13) \cdot a 12 \cdot\left(m 13^{2}+m 12^{2}+(m 13+m 12)^{2}\right)\right)+ \\
& +\left((1-a 13) \cdot(1-a 12) \cdot a 11 \cdot\left(m 13^{2}+m 12^{2}+m 11^{2}+(m 13+m 12+m 11)^{2}\right)\right) \text {; } \\
& E 2 A 2=\left(a 23 \cdot 2 \cdot m 23^{2}\right)+\left((1-a 23) \cdot a 22 \cdot\left(m 23^{2}+m 22^{2}+(m 23+m 22)^{2}\right)\right)+ \\
& +\left((1-a 23) \cdot(1-a 22) \cdot a 21 \cdot\left(m 23^{2}+m 22^{2}+m 21^{2}+(m 23+m 22+m 21)^{2}\right)\right) \text {; } \\
& E 3 A 2=\left(a 12 \cdot 2 \cdot m 12^{2}\right)+\left((1-a 12) \cdot a 11 \cdot\left(m 12^{2}+m 11^{2}+(m 12+m 11)^{2}\right)\right) ; \\
& E 4 A 2=\left(a 22 \cdot 2 \cdot m 22^{2}\right)+\left((1-a 22) \cdot a 21 \cdot\left(m 22^{2}+m 21^{2}+(m 22+m 21)^{2}\right)\right) ; \\
& E 5 A 2=\left(a 11.2 . m 11^{2}\right) ; \\
& E 6 A 2=\left(a 21.2 \cdot m 21^{2}\right) . \\
& E\left[W_{N}^{2} 1_{A}\right]=p i^{a}[05] \cdot\left(q_{1} \cdot E 1 A 2+q_{2} \cdot E 2 A 2\right)+p i^{a}[04] \cdot\left(q_{1} \cdot E 3 A 2+q_{2} \cdot E 4 A 2\right)+ \\
& +p i^{a}[03] .\left(q_{1} \cdot E 5 A 2+q_{2} \cdot E 6 A 2\right)
\end{aligned}
$$




\section{A.2 Algoritmo}

\section{A.2.1 Simulação dois tipos de clientes}

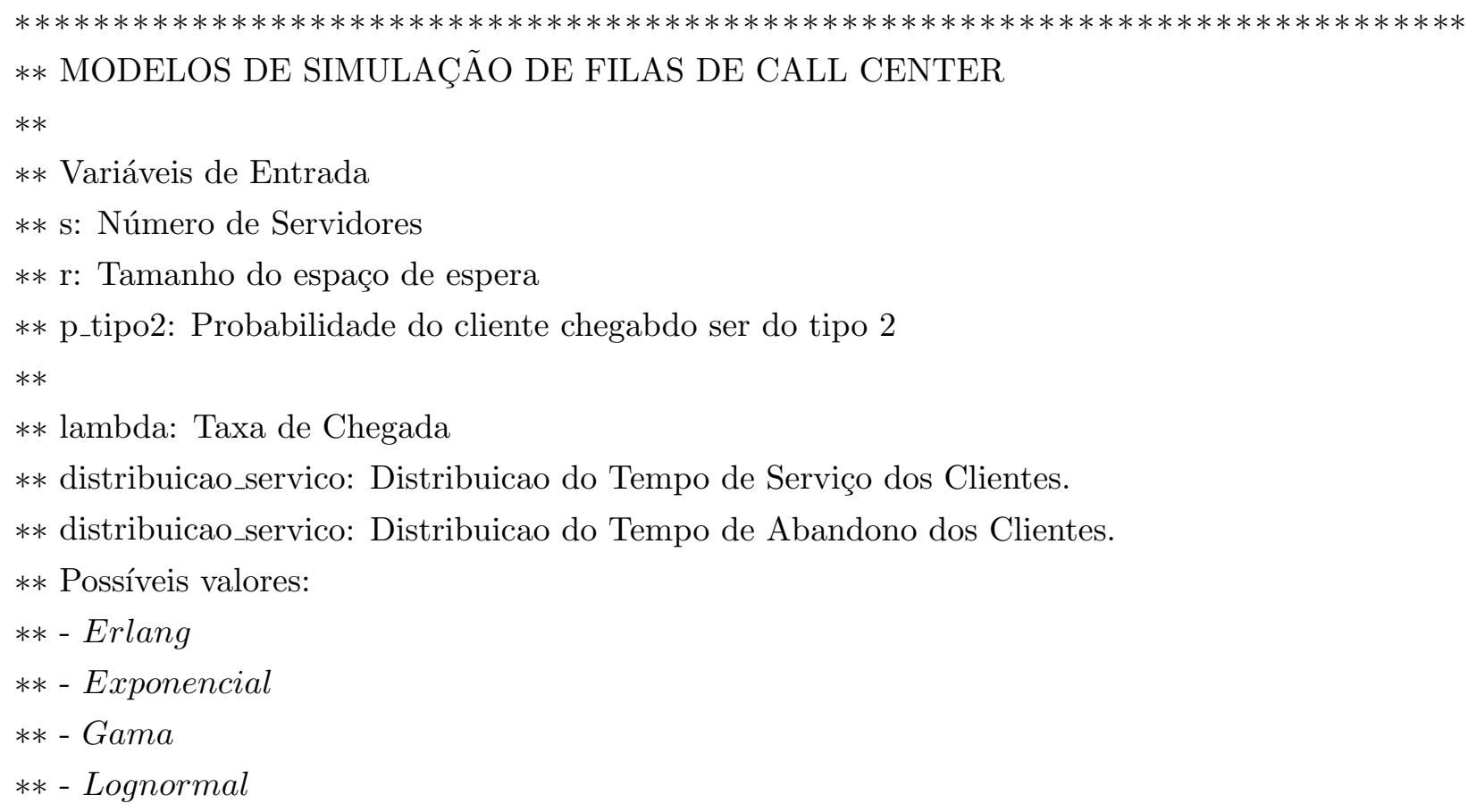


$* * *$ Simulação do processo de chegada

lambda $<-102$

n_clientes $<-40000$

$s<-100$

$r<-200$

$p<-0.7$

p_tipo $2<-1-p$

replicacoes $<-4$

metricas $<-\operatorname{array}(0, c(13$, replicacoes $))$

distribuicao_servico $<-$ distribuicao $<-$ distribuicao_servico $1<-$ Exponencial

distribuicao_abandono $<-$ distribuicao_abandono $1<-$ Exponencial

distribuicao_servico2 $<-$ Exponencial

distribuicao_abandono $2<-$ Exponencial

taxa_servico $<-$ taxa $<-$ taxa_servico $1<-1$

taxa_servico $2<-0.5$

taxa_abandono $<-$ taxa_abandono $1<-1$

taxa_abandono $2<-0.5$

for (replicacaoin 1 : replicacoes $)\{$

tempo_chegada $<-\operatorname{rexp}\left(n_{-}\right.$clientes, lambda $)$

tipo_cliente $<-$ rbinom $\left(n_{-}\right.$clientes, $1, p_{-}$tipo 2$)+1$

if (distribuicao_servico $1==$ "Exponencial" $)\{$

tempo_servico $1<-r e x p\left(n \_c l i e n t e s, r a t e=t a x a \_s e r v i c o 1\right)$

\}

if (distribuicao_servico $1==$ "Erlang" $)\{$

tempo_servico $1<-$ rgamma $\left(n \_c l i e n t e s\right.$, shape $=$ trunc $\left(k \_s e r v i c o 1\right)$, rate $=$ taxa_servico 1$)$

\}

if (distribuicao_servico $1==$ "Gama") \{

tempo_servico $1<-r g a m m a\left(n \_c l i e n t e s\right.$, shape $=$ forma_servico1,rate $=$ taxa_servico 1$)$

\} 


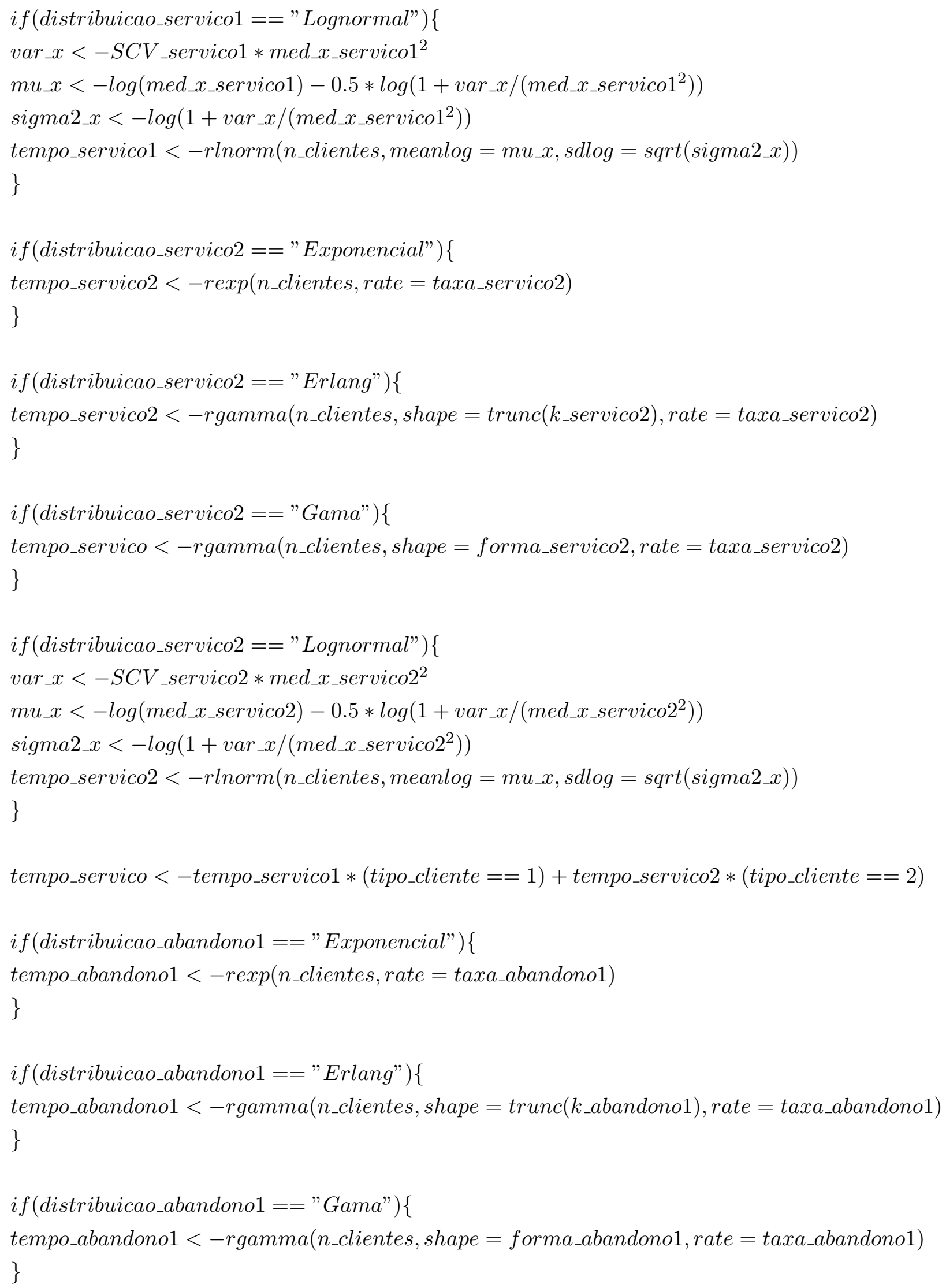




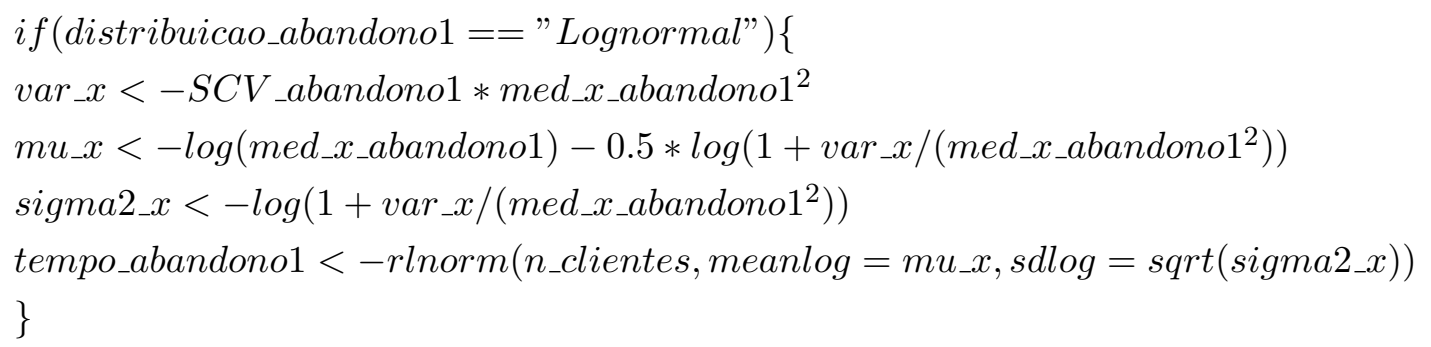

Exponencial, Erlang, Gama e Lognormal.

if (distribuicao_abandono $2==$ "Exponencial" $)\{$

tempo_abandono $2<-$ rexp $\left(n_{-}\right.$clientes, rate $=$taxa_abandono2 $)$

\}

if (distribuicao_abandono $2=="$ Erlang" $)\{$

tempo_abandono $2<-$ rgamma $\left(n \_c l i e n t e s\right.$, shape $=\operatorname{trunc}\left(k_{-} \_a b a n d o n o 2\right)$, rate $=$ taxa_abandono 2$)$

\}

if (distribuicao_abandono2 $==$ "Gama") \{

tempo_abandono $2<-r g a m m a\left(n \_c l i e n t e s\right.$, shape $=$ forma_abandono2, rate $=$ taxa_abandono 2$)$

\}

if (distribuicao_abandono $2=="$ Lognormal" $)\{$

$v a r \_x<-S C V \_a b a n d o n o 2 *$ med_x_abandono $2^{2}$

$m u \_x<-\log \left(m e d \_x \_a b a n d o n o 2\right)-0.5 * \log \left(1+v a r \_x /\left(m e d \_x \_a b a n d o n o 2^{2}\right)\right)$

sigma $2 \_x<-\log \left(1+\operatorname{var} \_x /\left(\operatorname{med} \_x \_a b a n d o n o 2^{2}\right)\right)$

tempo_abandono $2<-$ rlnorm $\left(n \_c l i e n t e s\right.$, meanlog $\left.=m u \_x, \operatorname{sdlog}=\operatorname{sqrt}\left(\operatorname{sigma} 2 \_x\right)\right)$

\}

tempo_abandono $<-$ tempo_abandono $1 *($ tipo_cliente $==1)+$

+ tempo_abandono $2 *($ tipo_cliente $==2)$

** Variável que controla os clientes atendidos pelos servidores no momento

** de ocorrência de determinado evento.

** Os valores de cada elemento do vetor pode ser 0 (caso não haja nenhum cliente),

** ou o índice do cliente (definido pela ordem de chegada na simulação).

clientes_servidores $<-r e p(0, s)$

** Variável que controla o tempo de espera de cada cliente antes de ser atendido

** Se o valor é igual a zero o cliente foi atendido sem espera. Esse tempo deve ser menor 
** ou igual ao tempo de abandono do cliente

tempo_espera_cliente $<-r e p\left(0, n_{-}\right.$clientes $)$

** Vetor que controla se determinado cliente foi servido (1) ou abandonou o sistema (0).

servido $<-r e p\left(1, n_{-}\right.$clientes $)$

** Vetor que contém o índice de todos os cliente que passaram pela fila e que não ** abandonaram o sistema.

fila $<-\operatorname{array}(0, c(1,2))$

** Vetor que armazena o tamanho da fila para cada tempo onde ocorre um evento

** (tamanho dependente das simulações).

tamanho_fila $<-0$

** Vetor que armazena a quantidade de clientes do tipo 1 na fila para cada tempo

** onde ocorre um evento.

tipo1_tamanho_fila $<-0$

** Variável que controla o Loop principal do sistema. Critério de término é quando ** o último cliente chega no sistema.

cliente $<-0$

** Controla o número de eventos ocorridos no sistema (Chegada, Final de Serviço ou Abandono) evento $<-0$

** Armazena o índice de todos os clientes que abandonaram o sistema

cliente_abandonaram $<-$ numeric $(0)$

** Armazena o índice de todos os clientes que foram recusados pelo sistema

cliente_recusados $<-$ numeric $(0)$

** Vetor que armazena a quantidade de clientes no sistema (servidores + fila)

** a cada ocorrência de um evento. Tamanho dependente das simulações.

cliente_sistema $<-$ numeric $(0)$

** Vetor que armazena a quantidade de clientes do tipo 1 no sistema (servidores + fila)

** a cada ocorrência de um evento. Tamanho dependente das simulações.

tipo1_cliente_sistema $<-$ numeric $(0)$ 
** Barra de progressão de cada iteração

$\operatorname{plot}\left(1, y l i m=\right.$ range $\left(0, n_{-}\right.$clientes $\left.)\right)$

ponto_tempo_chegada_todos_clientes $<-$ cumsum $($ tempo_chegada)

DESCOBRE_PRIMEIRO_EVENTO $<-$ function(tempo_evento_atual $)\{$

tipo_evento $<-"$-"

lista_clientes $<-$ numeric $(0)$

if $($ tamanho_fila[length $($ tamanho_fila $)]>0)\{$

lista_clientes $<-f i l a[($ length $($ fila $)-$ tamanho_fila[length $($ tamanho_fila $)]+1)$ : length $($ fila $)]$ \}

if $($ sum(clientes_servidores $)>0)\{$

lista_clientes $<-c($ lista_clientes, clientes_servidores[clientes_servidores! $=0])$

\}

for(clinlista_clientes $)\{$

ponto_tempo_chegada_cliente $<$ - ponto_tempo_chegada_todos_clientes[cl]

if $($ cl\%in\%clientes_servidores $)\{$

ponto_tempo_servico_cliente $<-$

ponto_tempo_chegada_cliente + tempo_espera_cliente $[c l]+$ tempo_servico $[c l]$

if (ponto_tempo_servico_cliente $<=$ tempo_evento_atual $)\{$

tipo_evento $<$-"terminoservico"

tempo_evento_atual $<-\min ($ tempo_evento_atual,ponto_tempo_servico_cliente)

cliente_alvo $<-c l$

\}

\}else\{

tempo_espera_cliente $[c l]<<-t e m p o \_e v e n t o \_a t u a l$ - ponto_tempo_chegada_cliente

if (tempo_espera_cliente $[\mathrm{cl}]>=$ tempo_abandono $[\mathrm{cl}])\{$

tempo_espera_cliente $[$ cl $]<-$ tempo_abandono $[$ cl $]$

tipo_evento $<$-"abandono"

tempo_evento_atual $<-$

min(tempo_evento_atual, ponto_tempo_chegada_cliente + tempo_abandono $[c l])$ 


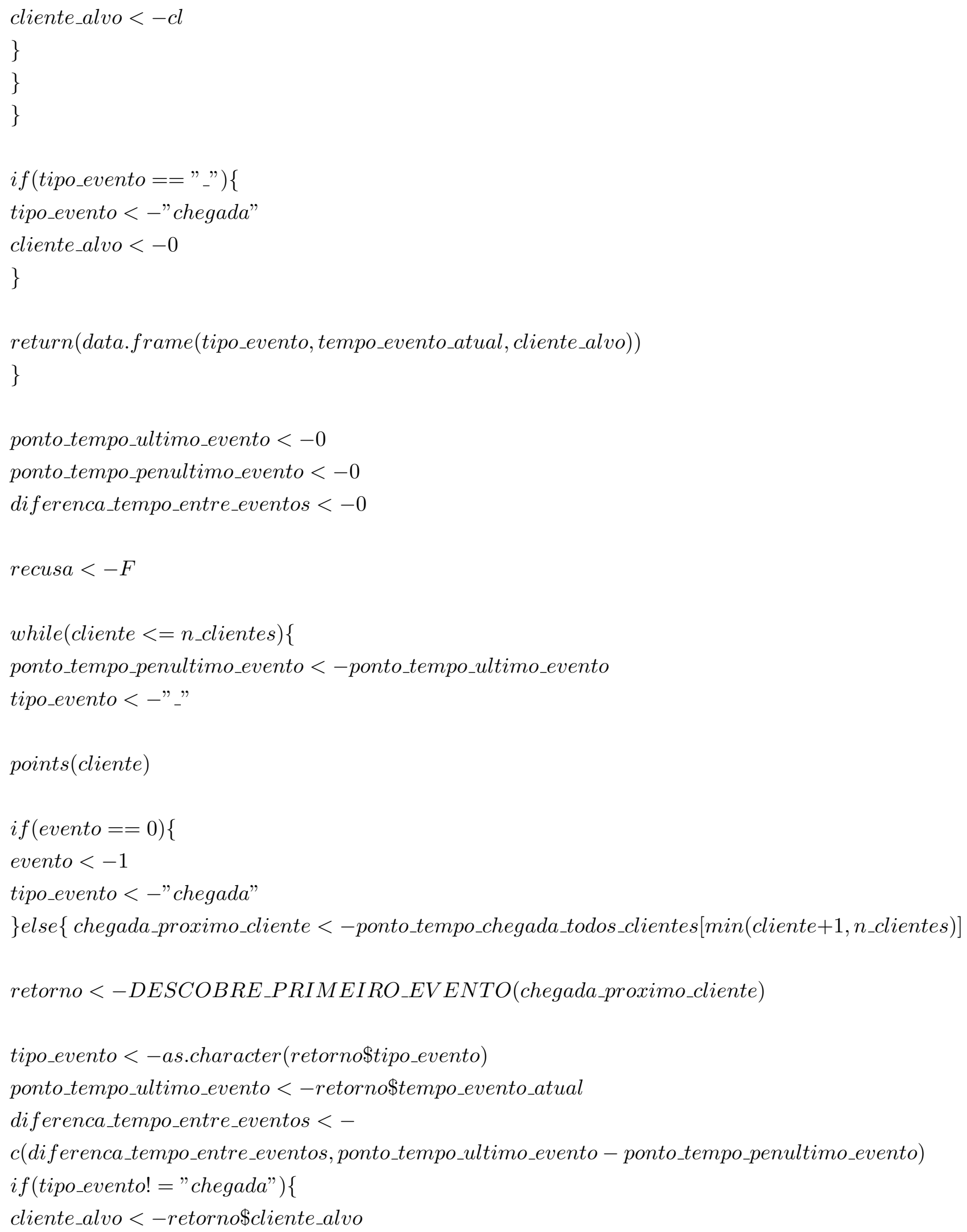


\}

\}

if $($ tipo_evento $=="$ chegada") \{

cliente $<-$ cliente +1

ponto_tempo_ultimo_evento $<$-ponto_tempo_chegada_todos_clientes[cliente]

diferenca_tempo_entre_eventos[length(diferenca_tempo_entre_eventos)] $<-$ ponto_tempo_ultimo_evento - ponto_tempo_penultimo_evento

if $($ length(clientes_servidores $[0 !=$ clientes_servidores $])<s)\{$

tempo_espera_cliente $[$ cliente $]<-0$

clientes_servidores[clientes_servidores $==0][1]<-$ cliente

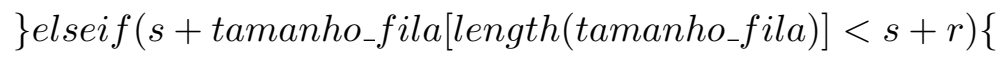

fila $<-c($ fila, cliente $)$

tamanho_fila $<-c($ tamanho_fila,tamanho_fila[length $($ tamanho_fila $)]+1)$

tipo1_tamanho_fila $<-$

c(tipo1_tamanho_fila,tipo1_tamanho_fila[length(tipo1_tamanho_fila)]+

(tipo_cliente $[$ cliente $]==1)$ )

\}else\{

cliente_recusados $<-c($ cliente_recusados, cliente $)$

recusa $<-T$

\}

\}

if $($ tipo_evento $=="$ terminoservico" $)\{$

if $($ tamanho_fila[length $($ tamanho_fila $)]>0)\{$

tamanho_fila $<-c($ tamanho_fila,tamanho_fila[length $($ tamanho_fila $)]-1)$

tipo1_tamanho_fila $<-$

c(tipo1_tamanho_fila,tipo1_tamanho_fila[length(tipo1_tamanho_fila $)]-($ tipo_cliente $[$ cliente_alvo $]==$ 1))

primeiro_cliente_fila $<-f i l a[$ length $($ fila $)-$ tamanho_fila[length(tamanho_fila $)]]$

tempo_espera_cliente $[$ primeiro_cliente_fila] $<-$

(ponto_tempo_ultimo_evento - ponto_tempo_chegada_todos_clientes[primeiro_cliente_fila])

if (tempo_espera_cliente $[$ primeiro_cliente_fila $]<=$ tempo_abandono[primeiro_cliente_fila $])\{$ 


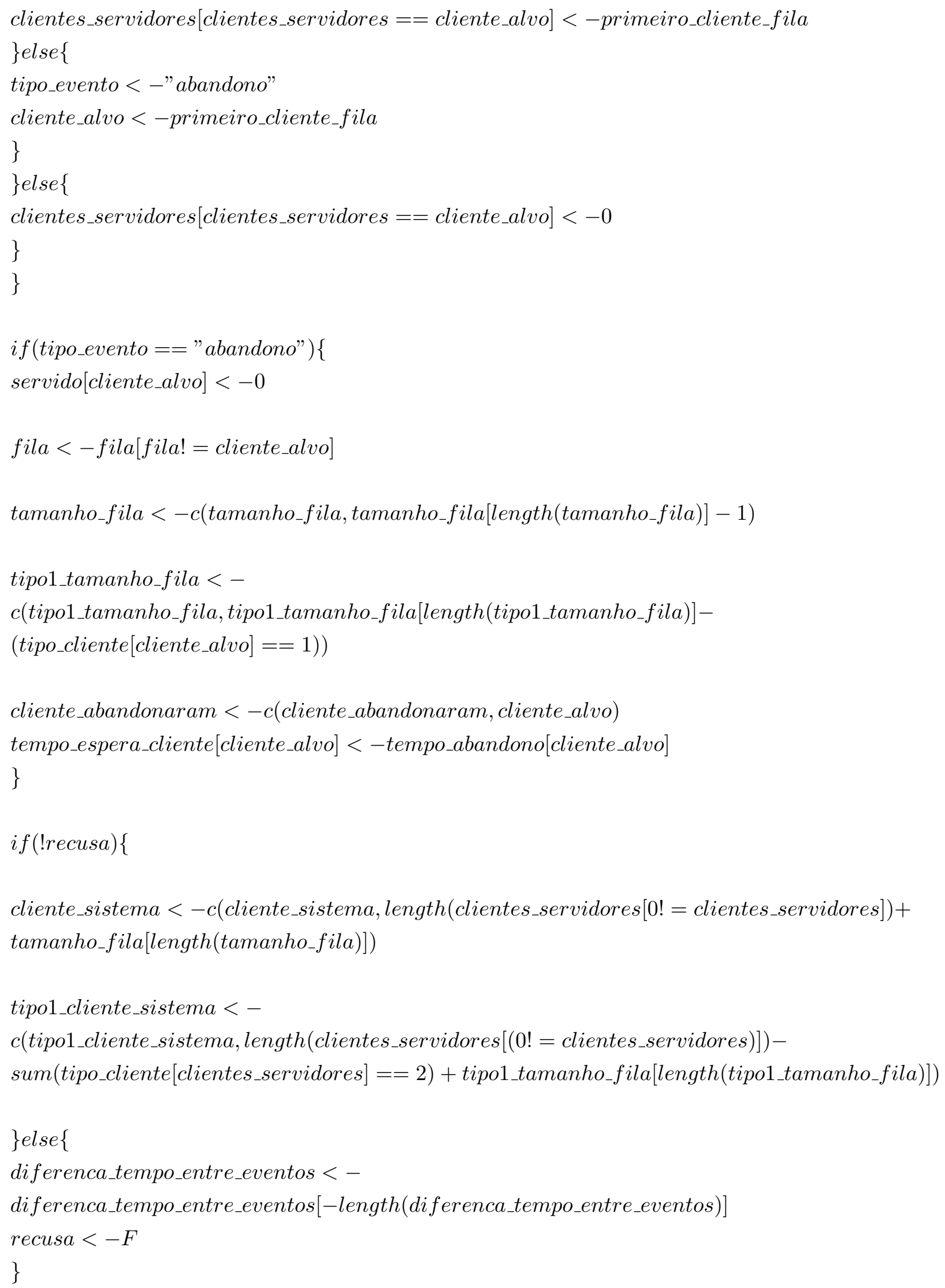


evento $<-$ evento +1

\}

if $($ length $($ cliente_recusados $)==0)\{$

cliente_recusados $<-1$

\}

inicio $<-1000$

fim $<-n_{-}$clientes

aux $<-($ tempo_espera_cliente[-cliente_recusados])[inicio : fim]

aux $<-a u x[$ !is.na $($ aux $)]$

selecao $<-($ tempo_espera_cliente[-cliente_recusados] $)[$ inicio : fim]

selecao $<-$ selecao[!is.na (selecao $)]$

$P W \_0<-$ length $($ aux $[$ selecao $==0]) /$ length $($ aux $)$

aux $<-($ servido[-cliente_recusados] $)[$ inicio $:$ fim $]$

aux $<-a u x[$ !is.na $($ aux $)]$

$P \_A<-$ length $($ aux $[$ aux $==0]) /$ length $(a u x)$

passo $<-\operatorname{trunc}($ length $($ cliente_sistema $))$

fim $<-$ trunc $($ length $($ cliente_sistema $))$

aux $<-$ tipo1_cliente_sistema[inicio $:$ length(tipo1_cliente_sistema $)]$

aux $<-a u x[$ !is.na $(a u x)]$

$E_{\_} N<-$ sum(cliente_sistema $*$ diferenca_tempo_entre_eventos, na.rm $=T$ )/

sum(diferenca_tempo_entre_eventos, na.rm $=T$ )

Var_Q $<-$

$\operatorname{sum}\left((\text { pmax }(\text { cliente_sistema }-s, 0)-\operatorname{mean}(\operatorname{pmax}(\text { cliente_sistema }-s, 0)))^{2} *\right.$

*diferenca_tempo_entre_eventos, na.rm $=T) /$ sum $($ diferenca_tempo_entre_eventos, na.rm $=T)$

$E \_Q<-\operatorname{sum}(\operatorname{pmax}($ cliente_sistema $-s, 0) *$ diferenca_tempo_entre_eventos, na.rm $=T) /$ sum(diferenca_tempo_entre_eventos, na.rm $=T$ )

fim $<-n_{-}$clientes 


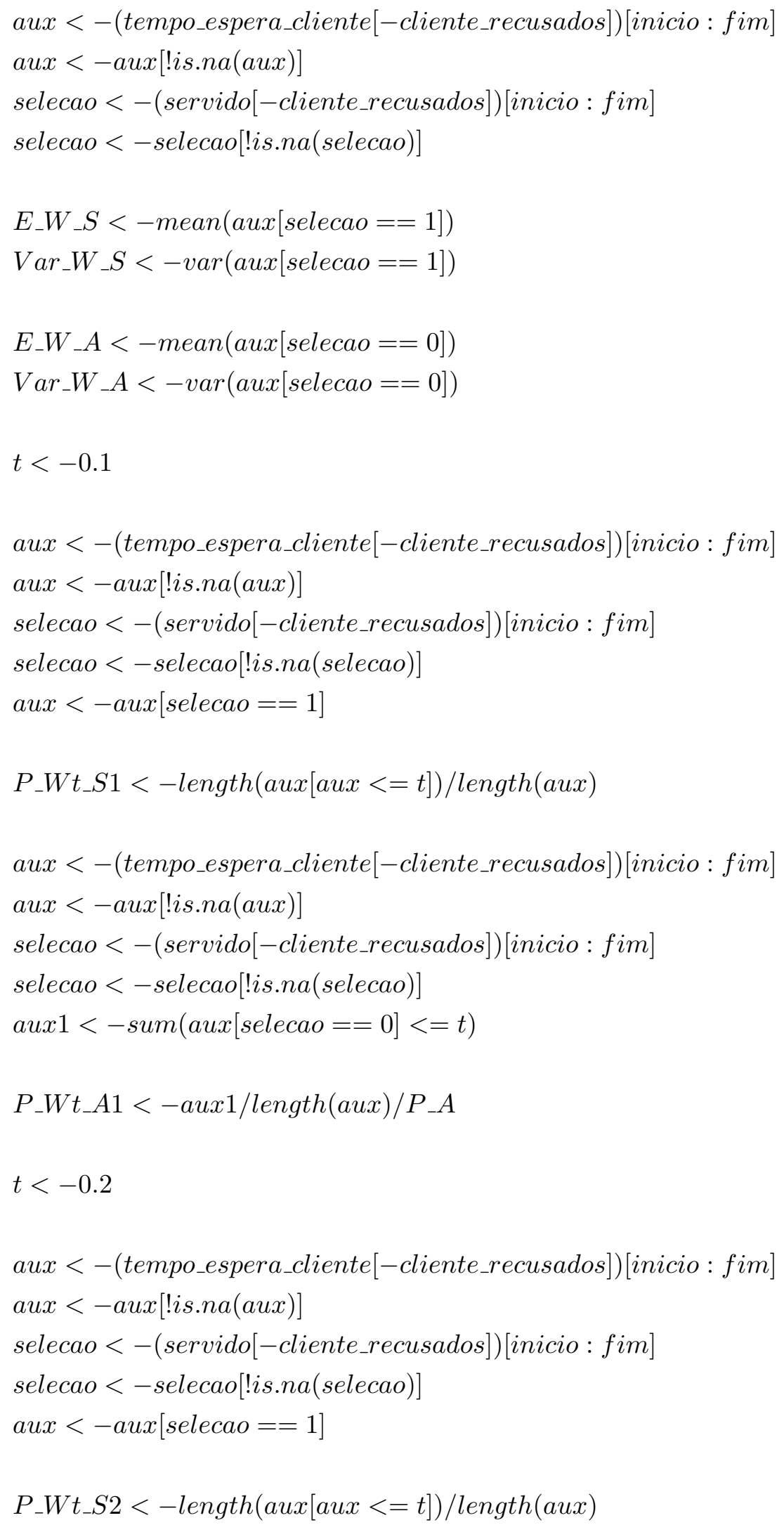


aux $<-($ tempo_espera_cliente[-cliente_recusados] $)[$ inicio $:$ fim]

aux $<-a u x[$ !is.na $(a u x)]$

selecao $<-($ servido[-cliente_recusados $])[$ inicio $:$ fim $]$

selecao $<-$ selecao $[$ !is.na $($ selecao $)]$

$\operatorname{aux} 1<-\operatorname{sum}(\operatorname{aux}[\operatorname{selecao}==0]<=t)$

$P_{-} W t_{-} A 2<-a u x 1 /$ length $($ aux $) / P_{-} A$

** Matriz que contém as métricas para cada replicação das simulações

metricas $[$, replicacao $]<-c\left(P W_{-} 0, P_{-} A, E_{-} Q, V a r_{-} Q, E_{-} N, E_{-} W \_S\right.$,

$\left.V a r_{-} W{ }_{-} S, E_{-} W{ }_{-} A, V a r_{-} W{ }_{-} A, P_{-} W t_{-} S 1, P_{-} W t_{-} A 1, P P_{-} W t_{-} S 2, P \_W t_{-} A 2\right)$

ic_metricas $<-\operatorname{array}(0, c(13,2))$

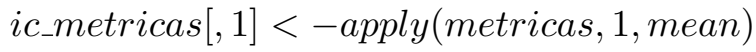

$i c \_m e t r i c a s[, 2]<-q t(0.975, d f=$ replicacoes -1$) * \operatorname{apply}($ metricas, 1, sd $) / \operatorname{sqrt}($ replicacoes $)$

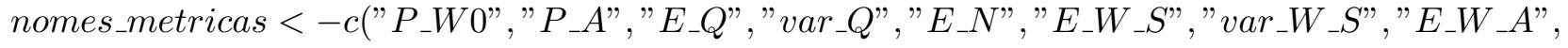
"var_W_A", "P_Wt_S_1", "P_Wt_A_1", "P_Wt_S_2", " $P_{-} W t_{-} A_{-} 2$ ")

resultados_metricas $<-\operatorname{array}(0, c(13,3))$

resultados_metricas[, 1] $<-$ nomes_metricas

resultados_metricas[,2] < -ic_metricas[, 1]

resultados_metricas $[, 3]<-i c \_$metricas $[, 2]$ 


\section{Referências Bibliográficas}

[1] V. Bolotin, Telephone circuit holding-time distributions, Proc. Internat. Teletraffic Congress (1994), 125-134.

[2] A. Brandt and M. Brandt, A two-queue priority system with impatience and its application to a call center, (1999).

[3] L. Brown, N. Gans, A. Mandelbaum, A. Sakov, H. Shen, S. Zeltyn, and L. Zhao, Tech. report.

[4] P. Chevalier, R. A. Shumsky, and N. Tabordon, Routing and staffing in large call centers with specialized and fully flexible servers, (2004).

[5] Z. Feldman, A. Mandelbaum, W. A. Massey, and W. Whitt, Staffing of time-varying queues to achieve time-stable performance, Management Science 54 (2008), no. 2, 324338.

[6] N. Gans, G. Koole, and A. Mandelbaum, Telephone call centers: Tutorial, review, and research prospects, Mgmt 5 (2003), 79-141.

[7] O. Garnett, A. Mandelbaum, and M. I. Reiman, Designing a call center with impatient customers, Manufacturing Service Oper. Management (2002), no. 4, 208227.

[8] L. Kleinrock, Queueing systems, vol. 1, 1975.

[9] M. N. Magalhães, Introducão à rede de filas, ABE - Associação Brasileira de Estatística, 1996, 12 SINAPE.

[10] _ Probabilidade e variáveis aleatórias, Edusp-Editora da Universidade de São Paulo, 2006, 2a. edição.

[11] A. Mandelbaum and R. Schwartz, Simulation experiments with $\mathrm{m} / \mathrm{g} / 100$ queues in the halfinwhitt (qed) regime, Technical report, The Technion, Israel (2002).

[12] A. Mandelbaum and S. Zeltyn, The impact of customers patience on delay and abandonment: some empirically-driven experiments with the $m / m / n+g$ queue, OR Spectrum 26 (2004), no. 3, $377-411$.

[13] L. P. Seelen, Tables for multi-server queues.

[14] W. Whitt, Improving service by informing customers about anticipated delays, Management Science 45 (1999), no. 2, 192-207.

[15] _ Engineering solution of a basic call-center model, Management Science 51 (2005), 221-235. 